math.QA/0606506

\title{
KAZHDAN-LUSZTIG-DUAL QUANTUM GROUP FOR LOGARITHMIC EXTENSIONS OF VIRASORO MINIMAL MODELS
}

\author{
B.L. FEIGIN, A.M. GAINUTDINOV, A.M. SEMIKHATOV, AND I.YU. TIPUNIN
}

\begin{abstract}
We derive and study a quantum group $\mathfrak{g}_{p, q}$ that is Kazhdan-Lusztig-dual to the $W$-algebra $\mathcal{W}_{p, q}$ of the logarithmic $(p, q)$ conformal field theory model. The algebra $\mathcal{W}_{p, q}$ is generated by two currents $W^{+}(z)$ and $W^{-}(z)$ of dimension $(2 p-1)(2 q-1)$, and the energy-momentum tensor $T(z)$. The two currents generate a vertex-operator ideal $\mathcal{R}$ with the property that the quotient $\mathcal{W}_{p, q} / \mathcal{R}$ is the vertex-operator algebra of the $(p, q)$ Virasoro minimal model. The number ( $2 p q)$ of irreducible $\mathfrak{g}_{p, q}$ representations is the same as the number of irreducible $\mathcal{W}_{p, q}$-representations on which $\mathcal{R}$ acts nontrivially. We find the center of $\mathfrak{g}_{p, q}$ and show that the modular group representation on it is equivalent to the modular group representation on the $\mathcal{W}_{p, q}$ characters and "pseudocharacters." The factorization of the $\mathfrak{g}_{p, q}$ ribbon element leads to a factorization of the modular group representation on the center. We also find the $\mathfrak{g}_{p, q}$ Grothendieck ring, which is presumably the "logarithmic" fusion of the $(p, q)$ model.
\end{abstract}

\section{CONTEnTs}

1. Introduction

Notation

2. The $\mathfrak{g}_{p_{+}, p_{-}}$quantum group, its representations, $q$-characters, and center

2.1. $\mathfrak{g}_{p_{+}, p_{-}}$from the double of the "two-screening" quantum group [22]

2.2. $\mathfrak{g}_{p_{+}, p_{-}}$-modules

2.3. The space of $q$-characters for $\mathfrak{g}_{p_{+}, p_{-}}$

2.4. The $\mathfrak{g}_{p_{+}, p_{-}}$center

3. Radford map of $\mathrm{Ch}\left(\mathfrak{g}_{p_{+}, p_{-}}\right)$

3.1. Radford map

3.2. Radford map of the $\gamma$ basis in $\operatorname{Ch}\left(\mathfrak{g}_{p_{+}, p_{-}}\right)$

4. The factorizable and ribbon structures for $\mathfrak{g}_{p_{+}, p_{-}}$

4.1. $M$-matrix

4.2. The Drinfeld map for $\mathfrak{g}_{p_{+}, p_{-}}$

4.3. The $\mathfrak{g}_{p_{+}, p_{-}}$ribbon element

5. $S L(2, \mathbb{Z})$-representations on the $\mathfrak{g}_{p_{+}, p_{-}}$center 29

5.1. $S L(2, \mathbb{Z})$-adapted bases in the center

5.2. Modular group action on the center

5.3. Comparison with the $S L(2, \mathbb{Z})$-representation on generalized characters of the $W$-algebra 34

5.4. Factorization of the $S L(2, \mathbb{Z})$ action

6. Conclusions

Appendix A. Some indecomposable $\mathfrak{g}_{p_{+}, p_{-}}$-modules

A.1. $\quad \mathcal{P}_{r, r^{\prime}}^{ \pm, \pm}$modules

A.2. Projective modules

B.1. Decomposition of the regular representation

B.2. Bimodule homomorphisms and the center

B.3. Explicit construction of the canonical basis in the center 
B.4. Some $\mathfrak{g}_{p_{+}, p_{-}}$calculations

\section{INTRODUCTION}

We consider quantum group counterparts of several important structures manifested in logarithmic $(p, q)$ conformal field theory models - examples of conformal field theory with nonsemisimple representation categories, which have been the subject of some attention from various standpoints [1, 2, 3, 4, 5, 6, 7, 8]. The logarithmic $(p, q)$ models can be quite interesting (for the $(3,2)$ model, there is evidence of its relation to the percolation problem [9, 10,11]), but their attractiveness is matched or, rather, counterbalanced by the difficulty of studying them directly, which arises primarily because the symmetries of such models are not the Virasoro algebra but its nonlinear extensions, some $W$-algebras (and in the $(3,2)$ model, for example, the leading pole in the operator product expansion of two currents generating the $W$-algebra is already of order 28). This highlights the importance of "indirect" methods, which concentrate around the Kazhdan-Lusztig correspondence [12].

The origin of the Kazhdan-Lusztig correspondence for logarithmic models can already be seen in their definition as kernels of screening operators acting in a free-field space (we recall that the rational minimal models are defined as the cohomology of screenings [13, 14]). The Kazhdan-Lusztig-dual quantum group $\mathfrak{g}$ is derived from the action of screenings that makes the space of states in conformal field theory into a bimodule - a module over the $W$-algebra and the quantum group $\mathfrak{g}$. In the $(p, 1)$ logarithmic models, this leads to the equivalence of braided quasitensor categories of the $W$-algebra representations and of the quantum group representations [5, 7].

Quantum group counterparts can be found not only for irreducible $W$-algebra representations/characters but also for a larger space $\mathcal{C}$ of torus amplitudes. We recall that in the nonsemisimple (logarithmic) case, $\mathcal{C}$ is spanned not only by the characters of irreducible representations but also by "generalized characters" (or "pseudocharacters") associated with some pseudotraces [3] (cf. [15, 16, 6]). On the quantum group side, similarly, irreducible $\mathfrak{g}$-modules provide only a subspace in the space $\mathrm{Ch}(\mathfrak{g})$ of $q$-characters ( $\mathrm{Ad}^{*}$-invariant functionals on the quantum group); spanning all of $\mathrm{Ch}(\mathfrak{g})$ by elements associated with representations requires taking pseudotraces associated with some indecomposable $\mathfrak{g}$-modules.

It can be expected in some generality that

$$
\mathcal{e}=\operatorname{Ch}(\mathfrak{g}),
$$

i.e., the space of torus amplitudes and the space of $q$-characters of the Kazhdan-Lusztigdual quantum group are isomorphic. Such an isomorphism becomes much more in- 
teresting if it extends to some structures defined on the respective spaces. The modular group action is a structure of major importance on $\mathcal{C}$. Remarkably, it is nicely matched by the general theory of the modular group action on ribbon factorizable quantum groups [17, 18, 19] (also see [20] for an application of this theory to the small quantum group). For factorizable quantum groups, there is an isomorphism (actually, of associative algebras [21])

$$
\mathrm{Ch}(\mathfrak{g}) \stackrel{\chi}{\longrightarrow} \mathrm{Z},
$$

which allows replacing $\mathrm{Ch}=\mathrm{Ch}(\mathfrak{g})$ with a somewhat more "tangible" object, the quantum group center Z. For the $(p, q)$ models, the $S L(2, \mathbb{Z})$ representation $\pi$ constructed on the center of the Kazhdan-Lusztig-dual quantum group in accordance with the recipe in [17, 18, 19] turns out to be equivalent to the $S L(2, \mathbb{Z})$ representation on the torus amplitudes, as we show in what follows (this has been known for the $(p, 1)$ logarithmic models [5]).

To formulate the result, we use the notation $p=p_{+}$and $q=p_{-}$for a fixed pair of coprime positive integers and set]

$$
\mathfrak{q}=e^{\frac{i \pi}{2 p_{+} p_{-}}}, \quad \mathfrak{q}_{+}=\mathfrak{q}^{2 p_{-}}=e^{\frac{i \pi}{p_{+}}}, \quad \mathfrak{q}_{-}=\mathfrak{q}^{2 p_{+}}=e^{\frac{i \pi}{p_{-}}} .
$$

On the conformal field theory side, the chiral algebra of the logarithmic $\left(p_{+}, p_{-}\right)$conformal field theory is a certain "triplet" $W$-algebra $\mathcal{W}_{p_{+}, p_{-}}$defined in [22]. The characters of its irreducible representations give rise to a $\frac{1}{2}\left(3 p_{+}-1\right)\left(3 p_{-}-1\right)$-dimensional representation $\mathrm{Z}_{\mathrm{cft}}$ of the modular group $S L(2, \mathbb{Z})$, evaluated as [22]

$$
\mathrm{Z}_{\mathrm{cft}}=R_{\text {min }} \oplus R_{\text {proj }} \oplus \mathbb{C}^{2} \otimes\left(R_{\square} \oplus R_{\square}\right) \oplus \mathbb{C}^{3} \otimes R_{\text {min }},
$$

where $\mathbb{C}^{2}$ is the standard two-dimensional representation, $\mathbb{C}^{3}$ is its symmetrized square, $R_{\text {min }}$ is the $\frac{1}{2}\left(p_{+}-1\right)\left(p_{-}-1\right)$-dimensional $S L(2, \mathbb{Z})$ representation on the characters of the $\left(p_{+}, p_{-}\right)$Virasoro minimal model, and $R_{\text {proj }}, R_{\square}$, and $R_{\square}$ are certain $S L(2, \mathbb{Z})$ representations of the respective dimensions $\frac{1}{2}\left(p_{+}+1\right)\left(p_{-}+1\right), \frac{1}{2}\left(p_{+}+1\right)\left(p_{-}-1\right)$, and $\frac{1}{2}\left(p_{+}-1\right)\left(p_{-}+1\right)$ (see [22] for the precise formulas).

On the quantum group side, the quantum group $\mathfrak{g}_{p_{+}, p_{-}}$that is Kazhdan-Lusztig-dual to the $\left(p_{+}, p_{-}\right)$logarithmic conformal field theory is the quotient

$$
\mathfrak{g}_{p_{+}, p_{-}}=\frac{\overline{\mathcal{U}}_{Q_{+}} s \ell(2) \otimes \overline{\mathcal{U}}_{Q_{-}} s \ell(2)}{\left(K_{+}^{p_{+}}-K_{-}^{p_{-}}\right)}
$$

of the product of two restricted quantum groups $\overline{\mathcal{U}}_{Q_{ \pm}} s \ell(2)$ at the roots of unity $Q_{ \pm}=\mathfrak{q}_{ \pm}^{p_{\mp}}$ over the (Hopf) ideal generated by the central element $K_{+}^{p_{+}}-K_{-}^{p_{-}}$.

\subsection{Theorem.}

(1) The center $\mathrm{Z}$ of $\mathfrak{g}_{p_{+}, p_{-}}$is $\frac{1}{2}\left(3 p_{+}-1\right)\left(3 p_{-}-1\right)$-dimensional.

\footnotetext{
${ }^{1}$ The paper is somewhat overloaded with the " \pm -type" notation, but it allows saving some space by " \pm " formulas.
} 
(2) The $S L(2, \mathbb{Z})$ representation $\pi$ on $\mathbf{Z}$ factors into the product of three $S L(2, \mathbb{Z})$ representations,

$$
\pi(A)=\pi_{0}(A) \pi_{(+)}(A) \pi_{(-)}(A), \quad A \in S L(2, \mathbb{Z}),
$$

which pairwise commute (i.e., $\pi_{0}(A) \pi_{(+)}\left(A^{\prime}\right)=\pi_{(+)}\left(A^{\prime}\right) \pi_{0}(A), \pi_{0}(A) \pi_{(-)}\left(A^{\prime}\right)$ $=\pi_{(-)}\left(A^{\prime}\right) \pi_{0}(A)$, and $\left.\pi_{(+)}(A) \pi_{(-)}\left(A^{\prime}\right)=\pi_{(-)}\left(A^{\prime}\right) \pi_{(+)}(A)\right)$.

(3) The center decomposes with respect to this product as

$\mathrm{Z}=R_{\text {proj }} \otimes 1 \otimes 1 \oplus R_{\square} \otimes \mathbb{C}^{2} \otimes 1 \oplus R_{\square} \otimes 1 \otimes \mathbb{C}^{2} \oplus R_{\text {min }} \otimes \mathbb{C}^{2} \otimes \mathbb{C}^{2}$,

where $\mathbb{C}^{2}$ is the standard two-dimensional representation, 1 is the trivial representation, $R_{\min }$ is the $\frac{1}{2}\left(p_{+}-1\right)\left(p_{-}-1\right)$-dimensional $S L(2, \mathbb{Z})$ representation on the characters of the $\left(p_{+}, p_{-}\right)$Virasoro minimal model, and $R_{\mathrm{proj}}, R_{\square}$, and $R_{\square}$ are those in (1.3).

(4) The representation

$$
R_{\text {proj }} \oplus R_{\square} \oplus R_{\square} \oplus R_{\text {min }}
$$

is the $S L(2, \mathbb{Z})$ representation on the Grothendieck ring of $\mathfrak{g}_{p_{+}, p_{-}}$.

(5) Moreover, the decomposition $\mathbb{C}^{2} \otimes \mathbb{C}^{2}=1 \oplus \mathbb{C}^{3}$ establishes an equivalence of the $S L(2, \mathbb{Z})$ representation on $\mathbf{Z}$ to the representation on the space of generalized $\mathcal{W}_{p_{+}, p_{-}}$-characters in $(1.3)$.

The $\mathfrak{g}_{p_{+}, p_{-}}$quantum group has $2 p_{+} p_{-}$irreducible representations, which we label as $\mathbf{X}_{r, r^{\prime}}^{ \pm}$with $1 \leqslant r \leqslant p_{+}$and $1 \leqslant r^{\prime} \leqslant p_{-}$.

1.2. Theorem. Multiplication in the $\mathfrak{g}_{p_{+}, p_{-}}$Grothendieck ring is given by

$$
\mathrm{X}_{r, r^{\prime}}^{\alpha} \mathbf{X}_{s, s^{\prime}}^{\beta}=\sum_{\substack{u=|r-s|+1 \\ \text { step }=2}}^{r+s-1} \sum_{\substack{u^{\prime}=\left|r^{\prime}-s^{\prime}\right|+1 \\ \text { step }=2}}^{r^{\prime}+s^{\prime}-1} \widetilde{\mathrm{X}}_{u, u^{\prime}}^{\alpha \beta}
$$

where

$$
\widetilde{\mathbf{X}}_{r, r^{\prime}}^{\alpha}= \begin{cases}\mathrm{X}_{r, r^{\prime}}^{\alpha}, & 1 \leqslant r \leqslant p_{+}, \\ \mathrm{X}_{2 p_{+}-r, r^{\prime}}^{\alpha}+2 \mathbf{X}_{r-p_{+}, r^{\prime}}^{-\alpha}, & 1 \leqslant r^{\prime} \leqslant p_{-}, \\ & p_{+}+1 \leqslant r \leqslant 2 p_{+}-1, \\ \mathrm{X}_{r, 2 p_{-}-r^{\prime}}^{\alpha}+2 \mathbf{X}_{r, r^{\prime}-p_{-}}^{-\alpha}, & 1 \leqslant r^{\prime} \leqslant p_{-}, \\ \mathrm{X}_{2 p_{+}-r, 2 p_{-}-r^{\prime}}^{\alpha}+2 \mathbf{X}_{2 p_{+}-r, r^{\prime}-p_{-}}^{-\alpha} & 1 \leqslant r \leqslant p_{+}, \\ +2 \mathbf{X}_{r-p_{+}, 2 p_{-}-r^{\prime}}^{-\alpha}+4 \mathbf{X}_{r-p_{+}, r^{\prime}-p_{-}}^{\alpha}, & p_{-}+1 \leqslant r^{\prime} \leqslant 2 p_{-}-1, \\ & p_{+}+1 \leqslant r \leqslant 2 p_{+}-1, \\ & p_{-}+1 \leqslant r^{\prime} \leqslant 2 p_{-}-1 .\end{cases}
$$


We also characterize this ring as a quotient of $\mathbb{C}[x, y]$ (see 4.2.7). Its interpretation as a $\mathcal{W}_{p_{+}, p_{-}}$fusion ring is discussed in [22].

To derive the Kazhdan-Lusztig-dual quantum group for the logarithmic $\left(p_{+}, p_{-}\right)$conformal field theory models, we follow the strategy proposed in [5] for $(p, 1)$ models. The starting point is the Hopf algebra $\mathcal{H}$ of screening operators in a free-field space. In the $(p, 1)$ case, $\mathcal{H}$ is a Taft Hopf algebra at a certain root of unity, and in the $\left(p_{+}, p_{-}\right)$case, $\mathcal{H}$ is constructed from two Taft algebras (taken at different roots of unity). We then pass to the Drinfeld double $D(\mathcal{H})$. From the conformal field theory standpoint, the double is a quantum group generated by the screening and contour-removal operators, the latter being just the dual to the former in Drinfeld's construction. But the doubling procedure also yields the dual $\kappa$ to the Cartan element $k$ in $\mathcal{H}$, and it is to be "eliminated" as $\kappa=k^{-1}$; this amounts to taking a quotient $\bar{D}$ of $D(\mathcal{H})$.

As any Drinfeld double, $D(\mathcal{H})$ is quasitriangular, i.e., has a universal $R$-matrix $R \in$ $D(\mathcal{H}) \otimes D(\mathcal{H})$. It follows that $\bar{D}$ is still quasitriangular, but the $M$-matrix $\bar{M}={ }^{t} \bar{R} \bar{R}$ turns out to be an element not of $\bar{D} \otimes \bar{D}$ but of $\mathfrak{g}_{p_{+}, p_{-}} \otimes \mathfrak{g}_{p_{+}, p_{-}}$, where (for the $\left(p_{+}, p_{-}\right)$ model specifically) $\mathfrak{g}_{p_{+}, p_{-}}$is the subalgebra in $\bar{D}$ generated by $K \equiv k^{2}$ and the other $\bar{D}$ generators. This $\mathfrak{g}_{p_{+}, p_{-}}$can be alternatively described as in (1.4).

This Kazhdan-Lusztig-dual quantum group $\mathfrak{g}_{p_{+}, p_{-}}$is not quasitriangular, but we have a pair $\mathfrak{g}_{p_{+}, p_{-}} \subset \bar{D}$ of quantum groups, one of which has an $M$-matrix but not an $R$ matrix, and the other has an $R$-matrix but not a (nondegenerate) $M$-matrix. Abusing the terminology, we still refer to the $\mathfrak{g}_{p_{+}, p_{-}}$quantum group as factorizable (although it, e.g., is not unimodular). Such a "deficient factorizability" suffices for constructing a modular group action on the $\mathfrak{g}_{p_{+}, p_{-}}$center Z. In accordance with [17, 18, 19], the action of $\mathcal{S}=\left(\begin{array}{cc}0 & 1 \\ -1 & 0\end{array}\right) \in S L(2, \mathbb{Z})$ on $\mathrm{Z}$ is constructed in terms of the Drinfeld and Radford maps $\chi$ and $\widehat{\phi}$ and is given by composing one of these with the inverse of the other,

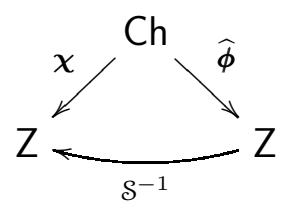

(the Radford map $\widehat{\phi}$ is the inverse compared with the standard definition). Next, $\mathcal{T}=$ $\left(\begin{array}{ll}1 & 1 \\ 0 & 1\end{array}\right) \in S L(2, \mathbb{Z})$ is represented on $\mathbf{Z}$ essentially by (multiplication with) the ribbon element,

$$
\mathrm{Z} \stackrel{v}{\longrightarrow} \mathrm{Z} \text {. }
$$

Here, too, the properties of $\mathfrak{g}_{p_{+}, p_{-}} \subset \bar{D}$ are such that the ribbon element $\boldsymbol{v}$ actually belongs to $\mathfrak{g}_{p_{+}, p_{-}}$(not surprisingly, in view of the relation between the $M$-matrix and the ribbon element). 
We prove Theorem 1.1 rather directly, by an explicit calculation relying on the existence of two special bases in the center, associated with the Drinfeld and Radford maps. Some "obvious" central elements that are related by $\mathcal{S}$ are given by the Radford and Drinfeld images of the ("quantum") traces over irreducible representations. 2 Adding some pseudotraces yields the "Radford" and "Drinfeld" bases in Z, with $\mathcal{S}$ mapping between them 3 The theorem eventually follows from studying how the ribbon element acts on the two bases.

The relation between the $S L(2, \mathbb{Z})$ representations in Claims 3 and 4 can be stated in terms of an automorphy factor, similarly to [2]. The factorization itself is related to the multiplicative Jordan decomposition of the ribbon element but goes somewhat further: the ribbon element factors as

$$
\boldsymbol{v}=\left(\sum_{\left(r, r^{\prime}\right) \in \mathcal{I}} e^{2 i \pi \Delta_{r, r^{\prime}}} \boldsymbol{e}\left(r, r^{\prime}\right)\right)\left(\mathbf{1}+\frac{1}{p_{+}} \chi^{\nwarrow \searrow}(1,1)\right)\left(\mathbf{1}+\frac{1}{p_{-}} \chi^{\nwarrow /}(1,1)\right) .
$$

The first factor is the semisimple part, constructed from the primitive idempotents $e\left(r, r^{\prime}\right)$ (weighted by exponentials of the conformal dimensions of $\mathcal{W}_{p_{+}, p_{-}}$primary fields in the $\left(p_{+}, p_{-}\right)$logarithmic conformal field theory model); in the other two factors, which are unipotent, $\chi^{\nwarrow \searrow}(1,1)$ and $\chi^{\not{\swarrow}}(1,1)$ are elements in the radical given by Drinfeld-map images of certain pseudotraces associated with indecomposable $\mathfrak{g}_{p_{+}, p_{-}}$representations in the full subcategory containing the trivial representation.

The rest of the paper is not difficult to describe, despite its length. We recall how the quantum group is derived from conformal field theory data in 2.1 and describe its representations in 2.2 (and Appendix $\mathrm{A}$ ), $q$-characters in 2.3 , and the center in 2.4 . We then travel along the diagram in (1.6) from top down, considering the $\widehat{\phi}$ arrow in Sec. 3 and the $\chi$ arrow in Sec. 4 . The Radford and Drinfeld maps $\widehat{\phi}$ and $\chi$ give two "S-adapted" bases in the center. It then remains to study how $\mathcal{T} \in S L(2, \mathbb{Z})$ acts on these bases. This amounts to studying the action of the ribbon element derived in 4.3 . We prove Theorem 1.1 in Sec. 5 ,

The basic ingredients for the $\widehat{\phi}$ map (and its inverse), the integral and cointegral, are found in 3.1.1, for the $\chi$ map, the required $M$-matrix is derived from the Drinfeld double in 4.1.1. The analysis of the $q$-characters $\mathrm{Ch}$ and the center $\mathrm{Z}$ relies on the study of representations, with an important role played by projective and some other indecomposable modules. These are studied in Appendix $\mathrm{A}$. In Appendix B, we detail the construction of the $\mathfrak{g}_{p_{+}, p_{-}}$center and collect some explicit calculations.

\footnotetext{
${ }^{2}$ From the standpoint of boundary conformal field theory, the $\widehat{\phi}$ map of the basis of characters and pseudocharacters in $\mathrm{Ch}$ yields the Ishibashi states, i.e., states with a certain "momentum." The $\chi$ map of the same elements in $\mathrm{Ch}$ yields the localized, i.e., Cardy states [23].

${ }^{3}$ The Drinfeld and Radford-map images of only the (traces over) irreducible representations do not span the entire center; this is an essential complication compared with the quantum $s \ell(2)$ in [20] and in [5].
} 
Notation. In $\mathbb{Z}\left[q, q^{-1}\right]$, we consider the $q$-integers and $q$-binomial coefficients

$$
[n]_{q}=\frac{q^{n}-q^{-n}}{q-q^{-1}}, \quad\left[\begin{array}{c}
m \\
n
\end{array}\right]_{q}=\frac{[m]_{q} !}{[n]_{q} ![m-n]_{q} !}, \quad[n]_{q} !=[1]_{q}[2]_{q} \ldots[n]_{q} .
$$

We then let

$$
[m]_{+}=[m]_{\mathfrak{q}_{+}^{p_{-}}}, \quad[m]_{-}=[m]_{\mathfrak{q}_{-}^{p_{+}}}, \quad\left[\begin{array}{c}
m \\
n
\end{array}\right]_{+}=\left[\begin{array}{c}
m \\
n
\end{array}\right]_{\mathfrak{q}_{+}^{p_{-}}}, \quad\left[\begin{array}{c}
m \\
n
\end{array}\right]_{-}=\left[\begin{array}{c}
m \\
n
\end{array}\right]_{\mathfrak{q}_{-}^{p_{+}}}
$$

denote their respective specializations at $\mathfrak{q}_{ \pm}^{p_{\mp}}$ (see (1.2)).

The fixed coprime pair $p_{+}$and $p_{-}$determines the four sets of pairs

$$
\mathcal{I}_{1}=\left\{\left(r, r^{\prime}\right) \mid 1 \leqslant r \leqslant p_{+}-1,1 \leqslant r^{\prime} \leqslant p_{-}-1, p_{-} r+p_{+} r^{\prime} \leqslant p_{+} p_{-}\right\}
$$

with $\left|\mathcal{I}_{1}\right|=\frac{1}{2}\left(p_{+}-1\right)\left(p_{-}-1\right)$,

$$
\begin{array}{ll}
\mathcal{I}_{\square}=\mathcal{I}_{1} \cup\left\{\left(r, p_{-}\right) \mid 1 \leqslant r \leqslant p_{+}-1\right\}, & \left|\mathcal{I}_{\square}\right|=\frac{1}{2}\left(p_{+}-1\right)\left(p_{-}+1\right), \\
\mathcal{I}_{\square}=\mathcal{I}_{1} \cup\left\{\left(p_{+}, r^{\prime}\right) \mid 1 \leqslant r^{\prime} \leqslant p_{-}-1\right\}, & \left|\mathcal{I}_{\square}\right|=\frac{1}{2}\left(p_{+}+1\right)\left(p_{-}-1\right),
\end{array}
$$

and

$$
\begin{aligned}
\mathcal{I}=\mathcal{I}_{1} \cup\left\{\left(r, p_{-}\right) \mid 1 \leqslant r \leqslant p_{+}-1\right\} \cup\left\{\left(p_{+}, r^{\prime}\right) \mid 1 \leqslant r^{\prime}\right. & \left.\leqslant p_{-}-1\right\} \\
& \cup\left\{\left(p_{+}, p_{-}\right)\right\} \cup\left\{\left(0, p_{-}\right)\right\}
\end{aligned}
$$

with $|\mathcal{I}|=\frac{1}{2}\left(p_{+}+1\right)\left(p_{-}+1\right)$. The sets $\mathcal{I}_{1}, \mathcal{I}_{\square}$, and $\mathcal{I}_{\square}$ are subsets of the extended Kac table, a $p_{+} \times p_{-}$rectangle, and $\mathcal{I}$ additionally contains the box conventionally labeled by $\left(0, p_{-}\right)$(it can be considered a "second copy" of the Steinberg box $\left(p_{+}, p_{-}\right)$). For $p_{+}=4$ and $p_{-}=7$, these are illustrated in Fig. 1 .

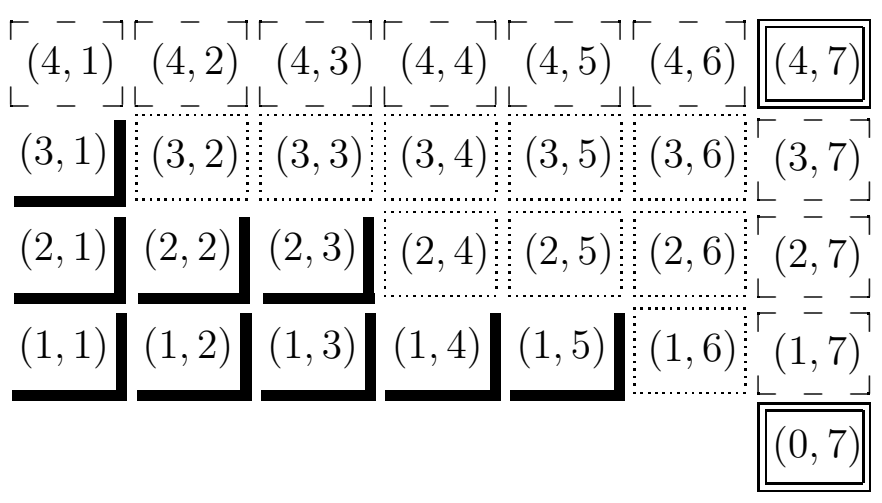

FIGURE 1. The four sets of pairs. The $\left(r, r^{\prime}\right)$ boxes constitute the set $\mathcal{I}_{1}$. The

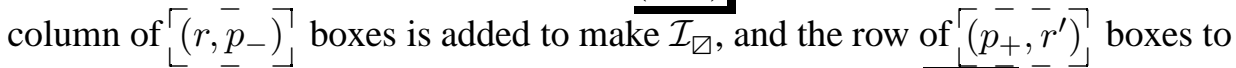
make $\mathcal{I}_{\square}$. Adding both the row and the column and the two $(,$,$) boxes makes$ the $\operatorname{set} \mathcal{I}$. 
2. THE $\mathfrak{g}_{p_{+}, p_{-}}$QUANTUM GROUP, ITS REPRESENTATIONS, $q$-CHARACTERS, AND CENTER

The quantum group corresponding to the logarithmic $\left(p_{+}, p_{-}\right)$conformal field theory model, $\mathfrak{g}_{p_{+}, p_{-}}$at an appropriate root of unity, is introduced in 2.1. We then consider its representations in 2.2. describe the space of $q$-characters in $\mathbf{2 . 3}$ and give the structure of the $\mathfrak{g}_{p_{+}, p_{-}}$center in $\mathbf{2 . 4}$

We refer the reader to [24, 25, 21, 26, 27] for the fundamental facts regarding Hopf algebra structures.

2.1. $\mathfrak{g}_{p_{+}, p_{-}}$from the double of the "two-screening" quantum group [22]. A "gateway" between the direct study of the logarithmic $\left(p_{+}, p_{-}\right)$model and its quantum group counterpart is provided by the screening operators $e_{+}$and $f_{-}$and a "Cartan" operator $k$ constructed from the free-field zero mode [22]. They act on the chiral sector of the space of states in the logarithmic model such that the relations

$$
\begin{gathered}
e_{+}^{p_{+}}=f_{-}^{p_{-}}=0, \quad k^{4 p_{+} p_{-}}=\mathbf{1}, \quad e_{+} f_{-}=f_{-} e_{+}, \\
k e_{+} k^{-1}=\mathfrak{q}_{+} e_{+}, \quad k f_{-} k^{-1}=\mathfrak{q}_{-}^{-1} f_{-}
\end{gathered}
$$

are satisfied. Let $\mathcal{H}$ denote the Hopf algebra with these generators and relations; its comultiplication, antipode, and counit (obtained by naturally combining those for the two Taft Hopf algebras generated by $\left(e_{+}, k\right)$ and $\left.\left(f_{-}, k\right)\right)$ are given in [22]. The PBW basis in $\mathcal{H}$ is

$$
\boldsymbol{e}_{j m n}=k^{j} e_{+}^{m} f_{-}^{n}, \quad 0 \leqslant j \leqslant 4 p_{+} p_{-}-1, \quad 0 \leqslant m \leqslant p_{+}-1, \quad 0 \leqslant n \leqslant p_{-}-1 .
$$

We next construct the Drinfeld double $D(\mathcal{H})$ of $\mathcal{H}$. This involves introducing generators $\kappa, f_{+}$, and $e_{-}$, "dual" to $k, e_{+}$, and $f_{-}$, by the relations

$$
\begin{gathered}
\left\langle\kappa, \boldsymbol{e}_{j m n}\right\rangle=\delta_{m, 0} \delta_{n, 0} \mathfrak{q}^{j}, \\
\left\langle f_{+}, \boldsymbol{e}_{j m n}\right\rangle=-\delta_{m, 1} \delta_{n, 0} \frac{\mathfrak{q}_{+}^{j}}{\mathfrak{q}_{+}^{p_{-}}-\mathfrak{q}_{+}^{-p_{-}}}, \quad\left\langle e_{-}, \boldsymbol{e}_{j m n}\right\rangle=-\delta_{m, 0} \delta_{n, 1} \frac{\mathfrak{q}_{-}^{-j}}{\mathfrak{q}_{-}^{p_{+}}-\mathfrak{q}_{-}^{-p_{+}}}
\end{gathered}
$$

Further, we eliminate $\kappa$, which is extraneous from the conformal field theory standpoint, by taking the quotient

$$
\bar{D}(\mathcal{H})=D(\mathcal{H}) /(k \kappa-\mathbf{1})
$$

over the Hopf ideal generated by the central element $k \kappa-1$. We then let $\mathfrak{g}_{p_{+}, p_{-}}$be the subalgebra in $\bar{D}(\mathcal{H})$ generated by (the images of) $e_{+}, f_{+}, e_{-}, f_{-}$, and $K=k^{2}$. It is shown in [22] that this it can be equivalently described as follows.

2.1.1. Proposition. $\mathfrak{g}_{p_{+}, p_{-}}$is the Hopf algebra generated by $e_{+}, f_{+}, e_{-}, f_{-}$, and $K$ with the relations

$$
e_{ \pm}^{p_{ \pm}}=f_{ \pm}^{p_{ \pm}}=0, \quad K^{2 p_{+} p_{-}}=\mathbf{1}
$$




$$
\begin{gathered}
K e_{ \pm} K^{-1}=\mathfrak{q}_{ \pm}^{2} e_{ \pm}, \quad K f_{ \pm} K^{-1}=\mathfrak{q}_{ \pm}^{-2} f_{ \pm}, \\
e_{+} e_{-}=e_{-} e_{+}, \quad f_{+} f_{-}=f_{-} f_{+}, \quad e_{+} f_{-}=f_{-} e_{+}, \quad e_{-} f_{+}=f_{+} e_{-}, \\
{\left[e_{+}, f_{+}\right]=\frac{K^{p_{-}-} K^{-p_{-}}}{\mathfrak{q}_{+}^{p_{-}-\mathfrak{q}_{+}^{-p_{-}}},} \quad\left[e_{-}, f_{-}\right]=\frac{K^{p_{+}}-K^{-p_{+}}}{\mathfrak{q}_{-}^{p_{+}}-\mathfrak{q}_{-}^{-p_{+}}}}
\end{gathered}
$$

and the Hopf algebra structure

$$
\begin{aligned}
& \Delta(K)=K \otimes K, \quad \Delta\left(e_{+}\right)=e_{+} \otimes \mathbf{1}+K^{p_{-}} \otimes e_{+}, \quad \Delta\left(f_{-}\right)=f_{-} \otimes \mathbf{1}+K^{-p_{+}} \otimes f_{-}, \\
& \Delta\left(f_{+}\right)=f_{+} \otimes K^{-p_{-}}+\mathbf{1} \otimes f_{+}, \quad \Delta\left(e_{-}\right)=e_{-} \otimes K^{p_{+}}+\mathbf{1} \otimes e_{-}, \\
& S(K)=K^{-1}, \quad S\left(e_{+}\right)=-K^{-p_{-}} e_{+}, \quad S\left(f_{-}\right)=-K^{p_{+}} f_{-}, \\
& S\left(f_{+}\right)=-f_{+} K^{p_{-}}, \quad S\left(e_{-}\right)=-e_{-} K^{-p_{+}}, \\
& \epsilon\left(e_{ \pm}\right)=\epsilon\left(f_{ \pm}\right)=0, \quad \epsilon(K)=1 \text {. }
\end{aligned}
$$

2.1.2. Furthermore, $\mathfrak{g}_{p_{+}, p_{-}}$can be described as the quotient (1.4) of the product of two restricted quantum groups $\overline{\mathcal{U}}_{Q_{ \pm}} s \ell(2)$ with $Q_{ \pm}=\mathfrak{q}_{ \pm}^{p_{\mp}}$ over the Hopf ideal generated by the central element $K_{+}^{p_{+}}-K_{-}^{p_{-}}$. By $\overline{\mathcal{U}}_{q} s \ell(2) \equiv \overline{\mathcal{U}}_{q}\langle e, f, K ; p\rangle$ at $q^{2 p}=1$, we mean the quantum group with the relations $e^{p}=f^{p}=0$ and $K^{2 p}=1$, the standard relations

$$
K e K^{-1}=q^{2} e, \quad K f K^{-1}=q^{-2} f, \quad[e, f]=\frac{K-K^{-1}}{q-\mathfrak{q}^{-1}}
$$

and the Hopf algebra structure given by

$$
\begin{gathered}
\Delta(K)=K \otimes K, \quad \Delta(e)=e \otimes \mathbf{1}+K \otimes e, \quad \Delta(f)=f \otimes K^{-1}+\mathbf{1} \otimes f, \\
S(K)=K^{-1}, \quad S(e)=-K^{-1} e, \quad S(f)=-f K .
\end{gathered}
$$

Indeed, because $p_{+}$and $p_{-}$are coprime, the $\mathfrak{g}_{p_{+}, p_{-}}$algebra in 2.1.1 is equivalently generated by $e_{+}, f_{+}, e_{-}, f_{-}$, and the elements

$$
K_{+}=K^{p_{-}}, \quad K_{-}=K^{p_{+}},
$$

From the formulas in 2.1.1, we then have

$$
K_{ \pm} e_{ \pm}=\mathfrak{q}_{ \pm}^{2 p_{\mp}} e_{ \pm} K_{ \pm}, \quad K_{ \pm} f_{ \pm}=\mathfrak{q}_{ \pm}^{-2 p_{\mp}} f_{ \pm} K_{ \pm}, \quad\left[e_{ \pm}, f_{ \pm}\right]=\frac{K_{ \pm}-K_{ \pm}^{-1}}{\mathfrak{q}_{ \pm}^{p_{\mp}}-\mathfrak{q}_{ \pm}^{-p_{\mp}}}
$$

and it follows in addition that $K_{ \pm} e_{\mp}=e_{\mp} K_{ \pm}$and $K_{ \pm} f_{\mp}=f_{\mp} K_{ \pm}$. The formulas for $\Delta$ and $S$ also "separate," e.g., $S\left(e_{+}\right)=-K_{+}^{-1} e_{+}$. The two $\overline{\mathcal{U}}_{q} s \ell(2)$ algebras are therefore $\overline{\mathcal{u}}_{\mathfrak{q}_{+}^{p_{-}}}\left\langle e_{+}, f_{+}, K_{+} ; p_{+}\right\rangle$and $\overline{\mathcal{u}}_{\mathfrak{q}_{-}^{p_{+}}}\left\langle f_{-}, e_{-}, K_{-}^{-1} ; p_{-}\right\rangle$. Clearly, the relations $K_{+}^{2 p_{+}}=K_{-}^{2 p_{-}}=$ $K_{+}^{p_{+}} K_{-}^{p_{-}}=1$ are satisfied by $K_{ \pm}$in (2.5).

2.2. $\mathfrak{g}_{p_{+}, p_{-}}$-modules. In what follows, we need the irreducible and projective $\mathfrak{g}_{p_{+}, p_{-}}{ }^{-}$ modules; on the way from the former to the latter, useful intermediate objects are the Verma modules. 
2.2.1. Irreducible modules. It is easy to see that there are $2 p_{+} p_{-}$irreducible finitedimensional $\mathfrak{g}_{p_{+}, p_{-}}$-modules. We label them as $\mathbf{X}_{r, r^{\prime}}^{ \pm}$, where $1 \leqslant r \leqslant p_{+}$and $1 \leqslant r^{\prime} \leqslant p_{-}$, with the highest-weight vector $\left|r, r^{\prime}\right\rangle^{ \pm}$of $\mathrm{X}_{r, r^{\prime}}^{ \pm}$defined by the relations

$$
e_{+}\left|r, r^{\prime}\right\rangle^{ \pm}=e_{-}\left|r, r^{\prime}\right\rangle^{ \pm}=0, \quad K\left|r, r^{\prime}\right\rangle^{ \pm}= \pm \mathfrak{q}_{+}^{r-1} \mathfrak{q}_{-}^{r^{\prime}-1}\left|r, r^{\prime}\right\rangle^{ \pm}
$$

It follows that $\operatorname{dim} \mathrm{X}_{r, r^{\prime}}^{ \pm}=r r^{\prime} . \quad \mathrm{X}_{1,1}^{+}$is the trivial module. The module $\mathrm{X}_{r, r^{\prime}}^{\alpha}$ is linearly spanned by elements $\left|r, r^{\prime}, n, n^{\prime}\right\rangle^{\alpha}, 0 \leqslant n \leqslant r-1$ and $0 \leqslant n^{\prime} \leqslant r^{\prime}-1$ (with $\left|r, r^{\prime}, 0,0\right\rangle^{ \pm} \equiv$ $\left.\left|r, r^{\prime}\right\rangle^{ \pm}\right)$, with the $\mathfrak{g}_{p_{+}, p_{-}}$-action given by

$$
\begin{aligned}
K\left|r, r^{\prime}, n, n^{\prime}\right\rangle^{\alpha} & =\alpha \mathfrak{q}_{+}^{r-1-2 n} \mathfrak{q}_{-}^{r^{\prime}-1-2 n^{\prime}}\left|r, r^{\prime}, n, n^{\prime}\right\rangle^{\alpha}, \\
e_{+}\left|r, r^{\prime}, n, n^{\prime}\right\rangle^{\alpha} & =\alpha^{p_{-}}(-1)^{r^{\prime}-1}[n]_{+}[r-n]_{+}\left|r, r^{\prime}, n-1, n^{\prime}\right\rangle^{\alpha}, \\
e_{-}\left|r, r^{\prime}, n, n^{\prime}\right\rangle^{\alpha} & =\alpha^{p_{+}}(-1)^{r-1}\left[n^{\prime}\right]_{-}\left[r^{\prime}-n^{\prime}\right]_{-}\left|r, r^{\prime}, n, n^{\prime}-1\right\rangle^{\alpha}, \\
f_{+}\left|r, r^{\prime}, n, n^{\prime}\right\rangle^{\alpha} & =\left|r, r^{\prime}, n+1, n^{\prime}\right\rangle^{\alpha}, \\
f_{-}\left|r, r^{\prime}, n, n^{\prime}\right\rangle^{\alpha} & =\left|r, r^{\prime}, n, n^{\prime}+1\right\rangle^{\alpha},
\end{aligned}
$$

where we set $\left|r, r^{\prime}, r, n^{\prime}\right\rangle^{\alpha}=\left|r, r^{\prime}, n, r^{\prime}\right\rangle^{\alpha}=\left|r, r^{\prime},-1, n^{\prime}\right\rangle^{\alpha}=\left|r, r^{\prime}, n,-1\right\rangle^{\alpha}=0$.

2.2.2. Verma modules. There are $2 p_{+} p_{-}$Verma modules, labeled as $\mathrm{V}_{r, r^{\prime}}^{ \pm}$with $1 \leqslant r \leqslant p_{+}$ and $1 \leqslant r^{\prime} \leqslant p_{-}$. First, these are the two Steinberg modules

$$
\mathrm{V}_{p_{+}, p_{-}}^{ \pm}=\mathrm{X}_{p_{+}, p_{-}}^{ \pm} \text {. }
$$

Next, for each $r=1, \ldots, p_{+}-1, r^{\prime}=1, \ldots, p_{-}-1$, and $a= \pm$, the Verma modules $\mathrm{V}_{r, p_{-}}^{a}$ and $\mathrm{V}_{p_{+}, r^{\prime}}^{a}$ can be described as the respective extensions

$$
\begin{aligned}
& 0 \rightarrow \mathrm{X}_{p_{+}-r, p_{-}}^{-a} \rightarrow \mathrm{V}_{r, p_{-}}^{a} \rightarrow \mathrm{X}_{r, p_{-}}^{a} \rightarrow 0 \\
& 0 \rightarrow \mathrm{X}_{p_{+}, p_{-}-r^{\prime}}^{-a} \rightarrow \mathrm{V}_{p_{+}, r^{\prime}}^{a} \rightarrow \mathrm{X}_{p_{+}, r^{\prime}}^{a} \rightarrow 0
\end{aligned}
$$

For consistency with the notation used in more complicated extensions below, we also write these extensions as
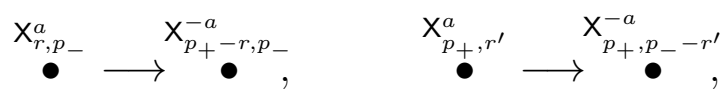

with the convention that arrows are directed toward submodules. 
Next, for $1 \leqslant r \leqslant p_{+}-1$ and $1 \leqslant r^{\prime} \leqslant p_{-}-1$, the Verma module $\mathrm{V}_{r, r^{\prime}}^{a}$ can be described as a second extension with the following structure of subquotients: 4$]$

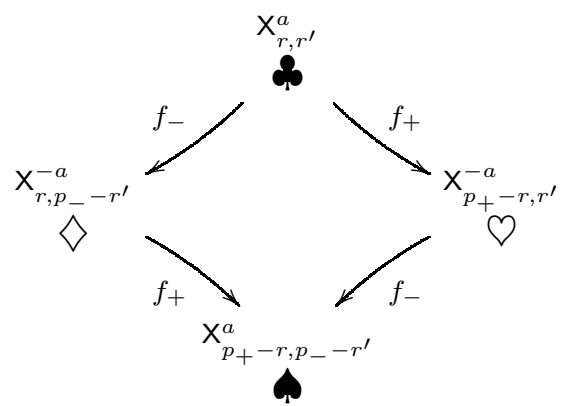

(a "four-suit" module). We note that $\operatorname{dim} \mathrm{V}_{r, r^{\prime}}^{a}=p_{+} p_{-}$.

2.2.3. Projective modules. The $2 p_{+} p_{-}$projective modules $\mathrm{P}_{r, r^{\prime}}^{ \pm}$, with $1 \leqslant r \leqslant p_{+}$and $1 \leqslant r^{\prime} \leqslant p_{-}$, are constructed in the standard way, as a projective cover of each irreducible module.

2.2.4. Proposition. The subquotient structure of the projective $\mathfrak{g}_{p_{+}, p_{-}}$-modules is as follows.

(1) $\mathrm{P}_{p_{+}, p_{-}}^{ \pm} \simeq \mathrm{X}_{p_{+}, p_{-}}^{ \pm}$are irreducible modules.

(2) For $1 \leqslant r \leqslant p_{+}-1$, the projective module $\mathrm{P}_{r, p_{-}}^{ \pm}$admits a filtration

$$
\mathrm{N}_{0}^{ \pm} \subset \mathrm{N}_{1}^{ \pm} \subset \mathrm{P}_{r, p_{-}}^{ \pm}
$$

where $\mathrm{N}_{0}^{ \pm} \simeq \mathrm{X}_{r, p_{-}}^{ \pm}, \mathrm{N}_{1}^{ \pm} / \mathrm{N}_{0}^{ \pm} \simeq \mathrm{X}_{p_{+}-r, p_{-}}^{\mp} \oplus \mathrm{X}_{p_{+}-r, p_{-}}^{\mp}$, and $\mathrm{P}_{r, p_{-}}^{ \pm} / \mathrm{N}_{1}^{ \pm} \simeq \mathrm{X}_{r, p_{-}}^{ \pm}$.

(3) For $1 \leqslant r^{\prime} \leqslant p_{-}-1$, the projective module $\mathrm{P}_{p_{+}, r^{\prime}}^{ \pm}$admits a filtration

$$
\mathrm{N}_{0}^{ \pm} \subset \mathrm{N}_{1}^{ \pm} \subset \mathrm{P}_{p_{+}, r^{\prime}}^{ \pm},
$$

where $\mathrm{N}_{0}^{ \pm} \simeq \mathrm{X}_{p_{+}, r^{\prime}}^{ \pm}, \mathrm{N}_{1}^{ \pm} / \mathrm{N}_{0}^{ \pm} \simeq \mathrm{X}_{p_{+}, p_{-}-r^{\prime}}^{\mp} \oplus \mathrm{X}_{p_{+}, p_{-}-r^{\prime}}^{\mp}$, and $\mathrm{P}_{p_{+}, r^{\prime}}^{ \pm} / \mathrm{N}_{1}^{ \pm} \simeq \mathrm{X}_{p_{+}, r^{\prime}}^{ \pm}$.

(4) For $1 \leqslant r \leqslant p_{+}-1$ and $1 \leqslant r^{\prime} \leqslant p_{-}-1$, the projective module $\mathrm{P}_{r, r^{\prime}}^{ \pm}$admits a filtration

$$
\mathrm{N}_{0}^{ \pm} \subset \mathrm{N}_{1}^{ \pm} \subset \mathrm{N}_{2}^{ \pm} \subset \mathrm{N}_{3}^{ \pm} \subset \mathrm{P}_{r, r^{\prime}}^{ \pm}
$$

where $\mathrm{N}_{0}^{ \pm} \simeq \mathrm{X}_{r, r^{\prime}}^{ \pm}, \quad \mathrm{N}_{1}^{ \pm} / \mathrm{N}_{0}^{ \pm} \simeq 2 \mathrm{X}_{r, p_{-}-r^{\prime}}^{\mp} \oplus 2 \mathrm{X}_{p_{+-r}, r^{\prime}}^{\mp}, \mathrm{N}_{2}^{ \pm} / \mathrm{N}_{1}^{ \pm} \simeq 4 \mathrm{X}_{p_{+}-r, p_{-}-r^{\prime}}^{ \pm} \oplus$ $2 \mathrm{X}_{r, r^{\prime}}^{ \pm}, \quad \mathrm{N}_{3}^{ \pm} / \mathrm{N}_{2}^{ \pm} \simeq 2 \mathrm{X}_{r, p_{-}-r^{\prime}}^{\mp} \oplus 2 \mathrm{X}_{p_{+}-r, r^{\prime}}^{\mp}$, and $\mathrm{P}_{r, r^{\prime}}^{ \pm} / \mathrm{N}_{3}^{ \pm} \simeq \mathrm{X}_{r, r^{\prime}}^{ \pm}$.

The structure of subquotients and the left $\mathfrak{g}_{p_{+}, p_{-}}$-action on $\mathrm{P}_{r, r^{\prime}}^{ \pm}$can be visualized with the aid of the diagram in Fig. 2. Their definition in terms of bases, whence 2.2.4 follows, is given in $\mathbf{A . 2}$

\footnotetext{
${ }^{4}$ In diagrams of this type, it is understood that the $\mathfrak{g}_{p_{+}, p_{-}}$-action on each irreducible representation is changed in agreement with the arrows connecting a given subquotient with others. For example, the lowest-weight vector of $\mathrm{X}_{r, r^{\prime}}^{a}$ is annihilated by $f_{-}$in the irreducible representation, but is mapped by $f_{-}$ into the highest-weight vector in $\mathrm{X}_{r, p_{-}-r^{\prime}}^{-a}$ in 2.7). With some more work, the remaining details of the $\mathfrak{g}_{p_{+}, p_{-}}$-action can then be reconstructed.
} 


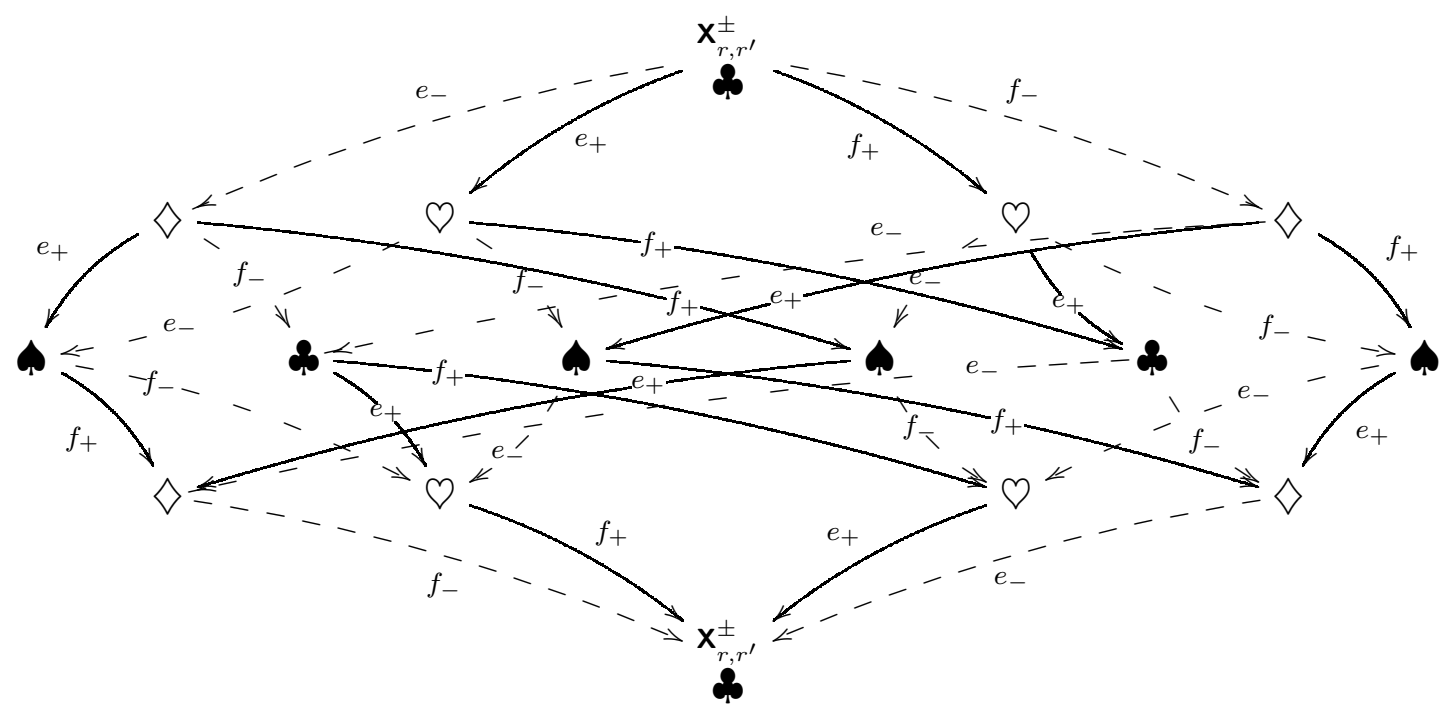

FIGURE 2. Subquotients of the projective module. We use the notation

$$
\begin{array}{ll}
\boldsymbol{\phi}=\mathrm{X}_{r, r^{\prime}}^{ \pm}, & \diamond=\mathrm{X}_{r, p_{-}-r^{\prime}}^{\mp}, \\
\curvearrowright=\mathrm{X}_{p_{+}-r, r^{\prime}}^{\mp}, & \boldsymbol{\phi}=\mathrm{X}_{p_{+}-r, p_{-}-r^{\prime}}^{ \pm}
\end{array}
$$

Continuous lines show the action of the $e_{+}$and $f_{+}$generators and dashed lines show the action of the $e_{-}$and $f_{-}$generators.

2.2.5. The $\mathfrak{g}_{p_{+}, p_{-}}$Grothendieck ring. Closely following the strategy in [5], we obtain Theorem 1.2 describing the Grothendieck ring structure of $\mathfrak{g}_{p_{+}, p_{-}}$. We do not repeat the standard steps leading to this statement, the derivation being totally similar to the one in [5]; it relies on a property of tensor products with Verma modules and simple explicit calculations for two-dimensional representations. The $\mathfrak{g}_{p_{+}, p_{-}}$Grothendieck ring is generated by $X_{1,2}^{+}$and $X_{2,1}^{+}$. In 4.2.6, it is further characterized as a polynomial quotient ring.

2.3. The space of $q$-characters for $\mathfrak{g}_{p_{+}, p_{-}}$. We now use irreducible and projective modules to introduce a basis in the space $\mathrm{Ch}=\mathrm{Ch}\left(\mathfrak{g}_{p_{+}, p_{-}}\right)$of $q$-characters of $\mathfrak{g}_{p_{+}, p_{-}}$. In subsequent sections, this basis is mapped into the center, to produce two distinguished bases there.

2.3.1. For a Hopf algebra $A$, the space $\mathrm{Ch}=\mathrm{Ch}(A)$ of $q$-characters is defined as

$$
\begin{aligned}
\operatorname{Ch}(A)=\left\{\beta \in A^{*} \mid \operatorname{Ad}_{x}^{*}(\beta)=\right. & \epsilon(x) \beta \quad \forall x \in A\} \\
& =\left\{\beta \in A^{*} \mid \beta(x y)=\beta\left(S^{2}(y) x\right) \quad \forall x, y \in A\right\},
\end{aligned}
$$

where the coadjoint action $\operatorname{Ad}_{a}^{*}: A^{*} \rightarrow A^{*}$ is $\operatorname{Ad}_{a}^{*}(\beta)=\beta\left(\sum_{(a)} S\left(a^{\prime}\right) ? a^{\prime \prime}\right), a \in A$, $\beta \in A^{*}$.

In what follows, we need the so-called balancing element $\boldsymbol{g} \in A$ that satisfies [21]

$$
\Delta(\boldsymbol{g})=\boldsymbol{g} \otimes \boldsymbol{g}, \quad S^{2}(x)=\boldsymbol{g} x \boldsymbol{g}^{-1}
$$


for all $x \in A$. For $A=\mathfrak{g}_{p_{+}, p_{-}}, \boldsymbol{g}=K^{p_{+}-p_{-}}$, as we calculate in (3.6) below.

2.3.2. Irreducible representation traces. The space of $q$-characters contains a homomorphic image of the Grothendieck ring under the $q$-trace: for any $A$-module $\mathrm{X}$,

$$
\mathrm{qCh}_{\mathbf{X}} \equiv \operatorname{Tr}_{\mathbf{X}}\left(\boldsymbol{g}^{-1} ?\right) \in \mathrm{Ch}(A),
$$

where $\boldsymbol{g}$ is the balancing element [21]. For $A=\mathfrak{g}_{p_{+}, p_{-}}$, we thus have a $2 p_{+} p_{-}$-dimensional subspace in Ch spanned by $q$-traces over irreducible modules, i.e., by

$$
\begin{aligned}
& \gamma^{ \pm}\left(r, r^{\prime}\right): x \mapsto \operatorname{Tr}_{\mathrm{X}_{r, r^{\prime}}^{ \pm}}\left(\boldsymbol{g}^{-1} x\right), \quad 1 \leqslant r \leqslant p_{+}, \quad 1 \leqslant r^{\prime} \leqslant p_{-}, \\
& \gamma^{ \pm}\left(r, r^{\prime}\right) \in \text { Ch. }
\end{aligned}
$$

2.3.3. Pseudotraces. The space of $q$-characters $\operatorname{Ch}\left(\mathfrak{g}_{p_{+}, p_{-}}\right)$is not spanned by $q$-traces over irreducible modules; it also contains "pseudotraces" associated with projective modules (in the Kazhdan-Lusztig context, they can be considered quantum group counterparts of pseudotraces [3]).

The strategy for constructing the pseudotraces is as follows. For any indecomposable module $\mathbb{P}$ and a map $\sigma: \mathbb{P} \rightarrow \mathbb{P}$, the functional

$$
\gamma: x \mapsto \operatorname{Tr}_{\mathbb{P}}\left(\boldsymbol{g}^{-1} x \sigma\right)
$$

is a $q$-character if and only if (cf. (2.8) $)$

$$
0=\gamma(x y)-\gamma\left(S^{2}(y) x\right) \equiv \operatorname{Tr}_{\mathbb{P}}\left(\boldsymbol{g}^{-1} x[y, \sigma]\right) .
$$

We now find maps $\sigma$ satisfying (2.11).

First, to choose $\mathbb{P}$, we note that the space of $q$-characters $\mathrm{Ch}\left(\mathfrak{g}_{p_{+}, p_{-}}\right)$is the center of the dual algebra $\mathfrak{g}_{p_{+}, p_{-}}^{*}$. To describe $\operatorname{Ch}\left(\mathfrak{g}_{p_{+}, p_{-}}\right)$, we can therefore use the isomorphism between the center of $\mathfrak{g}_{p_{+}, p_{-}}^{*}$ and the algebra of bimodule endomorphisms of the regular representation of $\mathfrak{g}_{p_{+}, p_{-}}^{*}$. As a $\mathfrak{g}_{p_{+}, p_{-}}^{*}$-bimodule, the regular representation is contragredient to the representation described in B.1. This means that $q$-characters are endomorphisms of the modules that are contragredient to projective $\mathfrak{g}_{p_{+}, p_{-}}$-modules. We therefore take $\mathbb{P}$ to be the projective $\mathfrak{g}_{p_{+}, p_{-}}$-module in one full subcategory, that is, the direct sum

$$
\mathbb{P}_{r, r^{\prime}}=\mathrm{P}_{r, r^{\prime}}^{+} \oplus \mathrm{P}_{p_{+}-r, r^{\prime}}^{-} \oplus \mathrm{P}_{r, p_{-}-r^{\prime}}^{-} \oplus \mathrm{P}_{p_{+}-r, p_{-}-r^{\prime}}^{+}
$$

(for $\left(r, r^{\prime}\right) \in \mathcal{I}_{1}$ ) of the four indecomposable projective $\mathfrak{g}_{p_{+}, p_{-}}$-modules $\mathrm{P}_{r, r^{\prime}}^{ \pm}$described in A.2. We then consider the maps

$$
\sigma: \mathbb{P}_{r, r^{\prime}} \rightarrow \mathbb{P}_{r, r^{\prime}}
$$

defined by its action on the corresponding basis vectors (see (A.5)): in each of the four modules in (2.12), $\sigma$ acts by zero on all elements in (A.5) except

$$
\sigma: \mathrm{b}_{n, n^{\prime}}^{\downarrow} \mapsto \alpha^{\uparrow} \mathrm{b}_{n, n^{\prime}}^{\uparrow}+\alpha^{\downarrow} \mathrm{b}_{n, n^{\prime}}^{\downarrow}+\beta^{\uparrow} \mathrm{t}_{n, n^{\prime}}^{\uparrow}+\beta^{\downarrow} \mathrm{t}_{n, n^{\prime}}^{\downarrow},
$$


with the coefficient $\alpha^{\uparrow}, \alpha^{\downarrow}, \beta^{\uparrow}$, and $\beta^{\downarrow}$ chosen arbitrarily in each module. To distinguish the basis elements in each of the four modules in (2.12), we introduce the second superscript and write

$$
\sigma: \mathbf{b}_{n, n^{\prime}}^{\downarrow, \bullet} \mapsto \alpha^{\uparrow, \bullet} \mathbf{b}_{n, n^{\prime}}^{\uparrow, \bullet}+\alpha^{\downarrow, \bullet} \mathbf{b}_{n, n^{\prime}}^{\downarrow, \bullet}+\beta^{\uparrow, \bullet} \mathbf{t}_{n, n^{\prime}}^{\uparrow, \bullet}+\beta^{\downarrow, \bullet} \mathbf{t}_{n, n^{\prime}}^{\downarrow, \bullet}
$$

where $\bullet \in\{\uparrow, \downarrow, \rightarrow, \leftarrow\}$ and the symbols where $\bullet$ "takes the value" $\uparrow$ correspond to the basis elements in $\mathrm{P}_{r, r^{\prime}}^{+}$, the elements where $\bullet=\leftarrow$ correspond to the basis elements in $\mathrm{P}_{r, p_{-} r^{\prime}}^{-}$, those where $\bullet=\rightarrow$ to the basis elements in $\mathrm{P}_{p_{+}-r, r^{\prime}}^{-}$, and those where $\bullet=\downarrow$ to the basis elements in $\mathrm{P}_{p_{+}-r, p_{-} r^{\prime}}^{+}$. This $\sigma$ map thus depends on $\left(\left(r, r^{\prime}\right)\right.$ and $)$ sixteen coefficients but, abusing the notation, we do not write these parameters explicitly.

For any such $\sigma$, we now define a functional on $\mathfrak{g}_{p_{+}, p_{-}}$as

$$
\gamma\left(r, r^{\prime}\right): x \mapsto \operatorname{Tr}_{\mathbb{P}_{r, r^{\prime}}}\left(\boldsymbol{g}^{-1} x \sigma\right) .
$$

A lengthy but straightforward calculation shows the following proposition.

2.3.4. Proposition. For $\left(r, r^{\prime}\right) \in \mathcal{I}_{1}$,

$$
\gamma\left(r, r^{\prime}\right) \in \mathrm{Ch}
$$

if and only if

$$
\begin{aligned}
& \alpha^{\uparrow, \uparrow}=\alpha^{\uparrow, \rightarrow}, \quad \alpha^{\uparrow, \downarrow}=\alpha^{\uparrow, \leftarrow}, \\
& \beta^{\downarrow, \uparrow}=\beta^{\downarrow, \leftarrow}, \quad \beta^{\downarrow, \downarrow}=\beta^{\downarrow, \rightarrow}, \\
& \beta^{\uparrow, \uparrow}=\beta^{\uparrow, \leftarrow}=\beta^{\uparrow, \rightarrow}=\beta^{\uparrow, \downarrow} .
\end{aligned}
$$

The proof is outlined in B.4.1.

2.3.5. The $\gamma$ basis. In what follows, we assume the relations in 2.3 .4 to be satisfied.

$$
\begin{aligned}
& \text { If } \alpha^{\uparrow, \uparrow}=\alpha^{\uparrow, \downarrow}=\beta^{\downarrow, \uparrow}=\beta^{\downarrow, \downarrow}=\beta^{\uparrow, \uparrow}=0 \text {, then } \\
& \begin{array}{r}
\gamma\left(r, r^{\prime}\right)=\alpha^{\downarrow, \uparrow} \gamma^{+}\left(r, r^{\prime}\right)+\alpha^{\downarrow, \leftarrow} \gamma^{-}\left(r, p_{-}-r^{\prime}\right) \\
\quad+\alpha^{\downarrow, \rightarrow} \gamma^{-}\left(p_{+}-r, r^{\prime}\right)+\alpha^{\downarrow, \downarrow} \gamma^{+}\left(p_{+}-r, p_{-}-r^{\prime}\right)
\end{array}
\end{aligned}
$$

is a linear combination of the $q$-traces over irredicible modules introduced in 2.3.2. To construct the other linearly independent $q$-characters, we take one of the coefficients $\alpha^{\uparrow, \bullet}$, $\beta^{\downarrow, \bullet}, \beta^{\uparrow} \bullet \bullet$ to be nonzero and thus define

$$
\begin{aligned}
& \gamma^{\not{ }}\left(r, r^{\prime}\right)=\gamma\left(r, r^{\prime}\right) \quad \text { for } \quad \alpha^{\uparrow, \downarrow}=\alpha^{\downarrow, \bullet}=\beta^{\downarrow} \bullet \beta^{\uparrow, \bullet}=0
\end{aligned}
$$

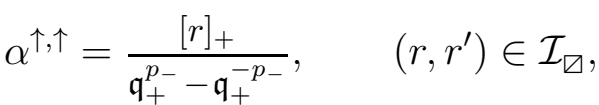

$$
\begin{aligned}
& \gamma^{\mathscr{X}}\left(p_{+}-r, p_{-}-r^{\prime}\right)=\gamma\left(r, r^{\prime}\right) \quad \text { for } \quad \alpha^{\uparrow, \uparrow}=\alpha^{\downarrow, \bullet}=\beta^{\downarrow, \bullet}=\beta^{\uparrow, \bullet}=0 \text {, }
\end{aligned}
$$

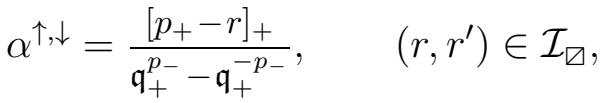


(2.17)

$$
\begin{aligned}
& \gamma^{\nwarrow \searrow}\left(r, r^{\prime}\right)=\gamma\left(r, r^{\prime}\right) \quad \text { for } \quad \alpha^{\downarrow, \bullet}=\alpha^{\uparrow, \bullet}=\beta^{\downarrow, \downarrow}=\beta^{\uparrow, \bullet}=0, \\
& \beta^{\downarrow, \uparrow}=\frac{\left[r^{\prime}\right]_{-}}{\mathfrak{q}_{-}^{p_{+}}-\mathfrak{q}_{-}^{-p_{+}}}, \quad\left(r, r^{\prime}\right) \in \mathcal{I}_{\square}, \\
& \gamma^{\nwarrow \searrow}\left(p_{+}-r, p_{-}-r^{\prime}\right)=\gamma\left(r, r^{\prime}\right) \quad \text { for } \quad \alpha^{\downarrow, \bullet}=\alpha^{\uparrow, \bullet}=\beta^{\downarrow, \uparrow}=\beta^{\uparrow, \bullet}=0 \\
& \beta^{\downarrow, \downarrow}=\frac{\left[p_{-}-r^{\prime}\right]_{-}}{\mathfrak{q}_{-}^{p_{+}}-\mathfrak{q}_{-}^{-p_{+}}}, \quad\left(r, r^{\prime}\right) \in \mathcal{I}_{\square}, \\
& \text { (2.19) } \gamma^{\uparrow}\left(r, r^{\prime}\right)=\gamma\left(r, r^{\prime}\right) \quad \text { for } \quad \alpha^{\downarrow, \bullet}=\alpha^{\uparrow, \bullet}=\beta^{\downarrow, \bullet}=0 \text {, } \\
& \beta^{\uparrow, \uparrow}=\alpha^{\uparrow, \uparrow} \beta^{\downarrow, \uparrow}, \quad\left(r, r^{\prime}\right) \in \mathcal{I}_{1} .
\end{aligned}
$$

For convenience, we introduce the maps $\sigma^{\mathbb{x}}\left(r, r^{\prime}\right)$ (with $\left.\left(r, r^{\prime}\right) \in \mathcal{I}_{\square}\right), \sigma^{\nwarrow \searrow}\left(r, r^{\prime}\right)$ (with $\left(r, r^{\prime}\right) \in \mathcal{I}_{\square}$ ), and $\sigma^{\uparrow}\left(r, r^{\prime}\right)$ (with $\left(r, r^{\prime}\right) \in \mathcal{I}_{1}$ ) obtained by substituting the respective sets of coefficients in (2.13).

We thus have $2 p_{+} p_{-}+\left(p_{+}-1\right) p_{-}+p_{+}\left(p_{-}-1\right)+\frac{1}{2}\left(p_{+}-1\right)\left(p_{-}-1\right)=\frac{1}{2}\left(3 p_{+}-1\right)\left(3 p_{-}-1\right)$ $q$-characters in the space $\mathrm{Ch}$ :

$$
\begin{aligned}
& \gamma^{+}\left(p_{+}, p_{-}\right), \quad \gamma^{\not{\alpha}}\left(r, p_{-}\right), \quad \gamma^{\Uparrow}\left(r, r^{\prime}\right), \quad \gamma^{\nwarrow \searrow}\left(p_{+}, r^{\prime}\right), \quad \gamma^{-}\left(p_{+}, p_{-}\right), \\
& 1 \leqslant r \leqslant p_{+}-1 \quad\left(r, r^{\prime}\right) \in \mathcal{I}_{1} \quad 1 \leqslant r^{\prime} \leqslant p_{-}-1 \\
& \gamma^{+}\left(r, p_{-}\right), \gamma^{-}\left(p_{+}-r, p_{-}\right), \quad \gamma^{\nwarrow}\left(r, r^{\prime}\right), \gamma^{\nwarrow \searrow}\left(r, r^{\prime}\right), \quad \gamma^{+}\left(p_{+}, r^{\prime}\right), \gamma^{-}\left(p_{+}, p_{-}-r^{\prime}\right), \\
& 1 \leqslant r \leqslant p_{+}-1 \quad 1 \leqslant r \leqslant p_{+}-1, \quad 1 \leqslant r^{\prime} \leqslant p_{-}-1 \\
& 1 \leqslant r^{\prime} \leqslant p_{-}-1 \\
& \gamma^{+}\left(r, r^{\prime}\right), \gamma^{-}\left(p_{+}-r, r^{\prime}\right), \gamma^{-}\left(r, p_{-}-r^{\prime}\right), \gamma^{+}\left(p_{+}-r, p_{-}-r^{\prime}\right) \text {. } \\
& \left(r, r^{\prime}\right) \in \mathcal{I}_{1}
\end{aligned}
$$

There are $\frac{1}{2}\left(3 p_{+}-1\right)\left(3 p_{-}-1\right)$ linearly independent elements in $\mathrm{Ch}$ thus obtained. In what follows, we also establish that $\operatorname{dim} Z=\frac{1}{2}\left(3 p_{+}-1\right)\left(3 p_{-}-1\right)$, with the consequence that the $\gamma$ listed above are a basis in Ch. We call it the $\gamma$-basis in what follows.

2.4. The $\mathfrak{g}_{p_{+}, p_{-}}$center. We now describe the structure of the center of $\mathfrak{g}_{p_{+}, p_{-}}$.

2.4.1. Theorem. The center $\mathrm{Z}$ of $\mathfrak{g}_{p_{+}, p_{-}}$is $\frac{1}{2}\left(3 p_{+}-1\right)\left(3 p_{-}-1\right)$-dimensional and decomposes into a direct sum of associative algebras as

$$
\mathbf{Z}=\mathfrak{I}_{p_{+}, p_{-}}^{(1)} \oplus \mathfrak{I}_{0, p_{-}}^{(1)} \oplus \bigoplus_{r=1}^{p_{+}-1} \mathfrak{B}_{r, p_{-}}^{(3)} \oplus \bigoplus_{r^{\prime}=1}^{p_{-}-1} \mathfrak{B}_{p_{+}, r^{\prime}}^{(3)} \oplus \bigoplus_{r, r^{\prime} \in \mathcal{I}_{1}} \mathfrak{A}_{r, r^{\prime}}^{(9)}
$$

where the dimension of each algebra is shown as a superscript. Furthermore,

- $\mathfrak{I}_{p_{+}, p_{-}}^{(1)}$ is spanned by a single idempotent $\boldsymbol{e}\left(p_{+}, p_{-}\right)$,

- $\mathfrak{I}_{0, p_{-}}^{(1)}$ is spanned by a single idempotent $\boldsymbol{e}\left(0, p_{-}\right)$, 
- each $\mathfrak{B}_{r, p_{-}}^{(3)}$ is spanned by the primitive idempotent $\boldsymbol{e}\left(r, p_{-}\right)$and the radical elements $\boldsymbol{v}^{\uparrow}\left(r, p_{-}\right)$and $\boldsymbol{v}^{\rightarrow}\left(r, p_{-}\right)$, on all of which $\boldsymbol{e}\left(r, p_{-}\right)$acts as identity and the other products vanish,

- each $\mathfrak{B}_{p_{+}, r^{\prime}}^{(3)}$ is spanned by the primitive idempotent $\boldsymbol{e}\left(p_{+}, r^{\prime}\right)$ and the radical elements $\boldsymbol{v}^{\uparrow}\left(p_{+}, r^{\prime}\right)$ and $\boldsymbol{v}^{\leftarrow}\left(p_{+}, r^{\prime}\right)$, on all of which $\boldsymbol{e}\left(p_{+}, r^{\prime}\right)$ acts as identity and the other products vanish,

- each $\mathfrak{A}_{r, r^{\prime}}^{(9)}$ is spanned by the primitive idempotent $\boldsymbol{e}\left(r, r^{\prime}\right)$ (acting as identity on $\left.\mathfrak{A}_{r, r^{\prime}}^{(9)}\right)$ and the radical elements $\boldsymbol{v}^{\nearrow}\left(r, r^{\prime}\right), \boldsymbol{v}^{\swarrow}\left(r, r^{\prime}\right), \boldsymbol{v}^{\nwarrow}\left(r, r^{\prime}\right), \boldsymbol{v}^{\searrow}\left(r, r^{\prime}\right), \boldsymbol{w}^{\uparrow}\left(r, r^{\prime}\right)$, $\boldsymbol{w}^{\rightarrow}\left(r, r^{\prime}\right), \boldsymbol{w}^{\downarrow}\left(r, r^{\prime}\right), \boldsymbol{w}^{\leftarrow}\left(r, r^{\prime}\right)$, which have the nonzero product 5

$$
\begin{array}{ll}
\boldsymbol{v}^{\nearrow}\left(r, r^{\prime}\right) \boldsymbol{v}^{\nwarrow}\left(r, r^{\prime}\right)=\boldsymbol{w}^{\uparrow}\left(r, r^{\prime}\right), & \boldsymbol{v}^{\nearrow}\left(r, r^{\prime}\right) \boldsymbol{v}^{\searrow}\left(r, r^{\prime}\right)=\boldsymbol{w}^{\rightarrow}\left(r, r^{\prime}\right), \\
\boldsymbol{v}^{\swarrow}\left(r, r^{\prime}\right) \boldsymbol{v}^{\nwarrow}\left(r, r^{\prime}\right)=\boldsymbol{w}^{\leftarrow}\left(r, r^{\prime}\right), & \boldsymbol{v}^{\swarrow}\left(r, r^{\prime}\right) \boldsymbol{v}^{\searrow}\left(r, r^{\prime}\right)=\boldsymbol{w}^{\downarrow}\left(r, r^{\prime}\right) .
\end{array}
$$

Pairwise products between elements in the radical other than those in (2.22) are identically zero.

The theorem is proved in Appendix $\mathbf{B}$

The $\frac{1}{2}\left(3 p_{+}-1\right)\left(3 p_{-}-1\right)$ elements $\boldsymbol{e}, \boldsymbol{v}$, and $\boldsymbol{w}$ are called the canonical central elements. A number of facts in what follows are established by decomposing various central elements with respect to the basis of the canonical central element. But because our next aim is to study the Radford map, it proves convenient to use a different basis in the center, given by the Radford-map images of the above $\gamma \in \mathrm{Ch}$ and actually obtained by certain mixing of the $e, v$, and $\boldsymbol{w}$ within each respective algebra in (2.21).

\section{RADFORD MAP OF $\mathrm{Ch}\left(\mathfrak{g}_{p_{+}, p_{-}}\right)$}

The $S L(2, \mathbb{Z})$-action on the center to be considered in Sec. [5involves the Radford and Drinfeld maps and a ribbon structure; the Radford map $\widehat{\phi}$ is studied in this section. We first find the necessary ingredients for $\widehat{\phi}$ and $\widehat{\phi}^{-1}$ (the cointegral and integral) and then evaluate the Radford images of the basis in Ch constructed in the previous section.

3.1. Radford map. We recall that for a Hopf algebra $A$, a right integral $\boldsymbol{\lambda}$ is a linear functional on $A$ satisfying

$$
(\boldsymbol{\lambda} \otimes \mathrm{id}) \Delta(x)=\boldsymbol{\lambda}(x) \mathbf{1}
$$

for all $x \in A$. If such a functional exists, it is unique up to multiplication by a nonzero constant. The left-right cointegral $\Lambda$ is an element in $A$ such that

$$
x \boldsymbol{\Lambda}=\boldsymbol{\Lambda} x=\epsilon(x) \boldsymbol{\Lambda}, \quad \forall x \in A .
$$

\footnotetext{
${ }^{5}$ The arrow notation is partly justified by the "momentum conservation law" satisfied in 2.22).

${ }^{6}$ For a unimodular $A^{*}$, which is the case with $A=\mathfrak{g}_{p_{+}, p_{-}}$.
} 
Whenever it exists, this element is unique up to multiplication by a nonzero constant. We also note that the cointegral gives an embedding of the trivial representation of $A$ in the regular bimodule $A$. We use the standard normalization $\boldsymbol{\lambda}(\boldsymbol{\Lambda})=1$.

Next, a comodulus $\boldsymbol{a}$ is an element in $A$ such that

$$
(\mathrm{id} \otimes \boldsymbol{\lambda}) \Delta(x)=\boldsymbol{\lambda}(x) \boldsymbol{a} .
$$

Whenever a square root of the comodulus exists, we have [21]

$$
\boldsymbol{g}^{2}=\boldsymbol{a},
$$

where $\boldsymbol{g} \in A$ is the balancing element.

The Radford map $\widehat{\phi}: A^{*} \rightarrow A$ and its inverse $\widehat{\phi}^{-1}: A \rightarrow A^{*}$ are given by

$$
\widehat{\boldsymbol{\phi}}(\beta)=\sum_{(\boldsymbol{\Lambda})} \beta\left(\boldsymbol{\Lambda}^{\prime}\right) \boldsymbol{\Lambda}^{\prime \prime}, \quad \widehat{\boldsymbol{\phi}}^{-1}(x)=\boldsymbol{\lambda}(S(x) ?) .
$$

Both $\widehat{\phi}$ and $\widehat{\phi}^{-1}$ intertwine the left actions of $A$ on $A$ and $A^{*}$, with the left $A$-module structures given by $a \rightarrow \beta=\beta\left(S(a)\right.$ ?) on $A^{*}$ and by the regular action on $A$, and similarly for the right actions [28, 29]. We use the hat for notational consistency in what follows (and swap $\widehat{\phi}$ and $\widehat{\phi}^{-1}$ compared with the commonly used notation).

In particular, $\widehat{\phi}$ gives an isomorphism of linear spaces $\widehat{\phi}: \mathrm{Ch} \rightarrow \mathrm{Z}$.

We now find the integral and cointegral and then evaluate $\widehat{\phi}$ on the $q$-characters constructed in 2.3 .

3.1.1. The integral, cointegral, and comodulus for $\mathfrak{g}_{p_{+}, p_{-}}$. A simple calculation in $\mathfrak{g}_{p_{+}, p_{-}}$shows that the right integral is given by

$$
\boldsymbol{\lambda}\left(e_{-}^{m^{\prime}} f_{+}^{n} K^{j} e_{+}^{m} f_{-}^{n^{\prime}}\right)=\frac{\mathfrak{q}_{+}^{2 p_{-}} \mathfrak{q}_{-}^{2 p_{+}}}{\zeta} \delta_{m^{\prime}, p_{-}-1} \delta_{n, p_{+}-1} \delta_{m, p_{+}-1} \delta_{n^{\prime}, p_{-}-1} \delta_{j, p_{+}-p_{-}} .
$$

Next, as is easy to verify, the left-right cointegral for $\mathfrak{g}_{p_{+}, p_{-}}$is given by the product of the cointegrals for the two $\bar{u}_{q} s \ell(2)$ in (1.4),

$$
\Lambda=\zeta f_{+}^{p_{+}-1} e_{+}^{p_{+}-1} f_{-}^{p_{-}-1} e_{-}^{p_{-}-1} \sum_{n=0}^{2 p_{+} p_{-}-1} K^{n}
$$

(which is at the same time the product of the cointegrals for the two $\overline{\mathcal{U}}_{q} s \ell(2)$ in (1.4), up to normalization). Factorizable ribbon quantum groups offer a "canonical" normalization of the Radford map (i.e., of the integral and cointegral) up a power of $i=\sqrt{-1}$, from the condition $\left.\mathcal{S}^{4}\right|_{\mathrm{z}}=$ id for $\mathcal{S}$ in (1.6), and we therefore set

$$
\zeta\left(\left[p_{+}-1\right]_{+} !\left[p_{-}-1\right]_{-} !\right)^{2}=\sqrt{\frac{p_{+} p_{-}}{2}} .
$$

We further calculate

$$
(\mathrm{id} \otimes \boldsymbol{\lambda}) \Delta\left(e_{-}^{p_{-}-1} f_{+}^{p_{+}-1} k^{2 p_{+}-2 p_{-}} e_{+}^{p_{+}-1} f_{-}^{p_{-}-1}\right)=\frac{\mathfrak{q}_{+}^{2 p_{-}} \mathfrak{q}_{-}^{2 p_{+}}}{\zeta} k^{4 p_{+}-4 p_{-}}
$$


which allows choosing the comodulus as $\boldsymbol{a}=K^{2 p_{+}-2 p_{-}}$. In accordance with (3.1), we then have the balancing element

$$
\boldsymbol{g}=K^{p_{+}-p_{-}} .
$$

3.2. Radford map of the $\gamma$ basis in $\mathrm{Ch}\left(\mathfrak{g}_{p_{+}, p_{-}}\right)$. Explicitly, the Radford map $\widehat{\boldsymbol{\phi}}: \mathrm{Ch} \rightarrow$ $\mathrm{Z}$ of the irreducible representation characters is given by

$$
\mathrm{X}_{r, r^{\prime}}^{ \pm} \mapsto \widehat{\boldsymbol{\phi}}^{ \pm}\left(r, r^{\prime}\right) \equiv \widehat{\boldsymbol{\phi}}\left(\mathrm{qCh}_{\mathrm{X}_{r, r^{\prime}}^{ \pm}}\right)=\sum_{(\boldsymbol{\Lambda})} \operatorname{Tr}_{\mathrm{X}_{r, r^{\prime}}^{ \pm}}\left(K^{p_{-}-p_{+}} \boldsymbol{\Lambda}^{\prime}\right) \boldsymbol{\Lambda}^{\prime \prime}, \quad \begin{aligned}
& 1 \leqslant r \leqslant p_{+}, \\
& 1 \leqslant r^{\prime} \leqslant p_{-} .
\end{aligned}
$$

Clearly, $\widehat{\phi}^{+}(1,1)=\Lambda$, in accordance with the fact that $\Lambda$ furnishes an embedding of the trivial representation $\mathrm{X}_{1,1}^{+}$into $\mathfrak{g}_{p_{+}, p_{-}}$. Because the Radford map $\mathrm{G}_{2 p_{+} p_{-}} \rightarrow \mathrm{Z}$ is a morphism of representations, it follows that the linear span of the $\widehat{\phi}^{ \pm}\left(r, r^{\prime}\right)$ is the annihilator of the radical in the center (see formulas below in 3.2.1).

The elements $\gamma^{\mathscr{x}}\left(r, r^{\prime}\right)$ are mapped under $\widehat{\phi}$ as

$$
\widehat{\boldsymbol{\phi}}\left(\gamma^{\not{\not}}\left(r, r^{\prime}\right)\right)=\widehat{\boldsymbol{\phi}}^{\not{\not}}\left(r, r^{\prime}\right)=\sum_{(\boldsymbol{\Lambda})} \operatorname{Tr}_{\mathbb{P}_{r, r^{\prime}}}\left(K^{p_{-}-p_{+}} \boldsymbol{\Lambda}^{\prime} \sigma^{\not{K}}\left(r, r^{\prime}\right)\right) \boldsymbol{\Lambda}^{\prime \prime}
$$

for all $1 \leqslant r \leqslant p_{+}-1$ and $1 \leqslant r^{\prime} \leqslant p_{-}$.

The elements $\gamma^{\nwarrow \searrow}\left(r, r^{\prime}\right)$ are mapped under $\widehat{\phi}$ as

$$
\widehat{\boldsymbol{\phi}}\left(\gamma^{\nwarrow \searrow}\left(r, r^{\prime}\right)\right)=\widehat{\boldsymbol{\phi}}^{\nwarrow \searrow}\left(r, r^{\prime}\right)=\sum_{(\boldsymbol{\Lambda})} \operatorname{Tr}_{\mathbb{P}_{r, r^{\prime}}}\left(K^{p_{-}-p_{+}} \boldsymbol{\Lambda}^{\prime} \sigma^{\nwarrow \searrow}\left(r, r^{\prime}\right)\right) \boldsymbol{\Lambda}^{\prime \prime},
$$

for all $1 \leqslant r \leqslant p_{+}$and $1 \leqslant r^{\prime} \leqslant p_{-}-1$.

Finally, the elements $\gamma^{\Uparrow}\left(r, r^{\prime}\right)$ with $\left(r, r^{\prime}\right) \in \mathcal{I}_{1}$ are mapped under $\widehat{\phi}$ as

$$
\widehat{\boldsymbol{\phi}}\left(\gamma^{\Uparrow}\left(r, r^{\prime}\right)\right)=\widehat{\boldsymbol{\phi}}^{\Uparrow}\left(r, r^{\prime}\right)=\sum_{(\boldsymbol{\Lambda})} \operatorname{Tr}_{\mathbb{P}_{r, r^{\prime}}}\left(K^{p_{-}-p_{+}} \boldsymbol{\Lambda}^{\prime} \sigma^{\Uparrow}\left(r, r^{\prime}\right)\right) \boldsymbol{\Lambda}^{\prime \prime} .
$$

3.2.1. Proposition. The Radford-map images

$$
\widehat{\phi}^{\bullet}\left(r, r^{\prime}\right)=\widehat{\phi}\left(\gamma^{\bullet}\left(r, r^{\prime}\right)\right), \quad \bullet=\uparrow, \ll, \ltimes \searrow,+,-,
$$

of the $\gamma$-basis in (2.20) are as follows.

(1) The Radford images of the irreducible representation traces $\gamma^{ \pm}\left(r, r^{\prime}\right), 1 \leqslant r \leqslant p_{+}$, $1 \leqslant r^{\prime} \leqslant p_{-}$, are given by

$$
\begin{aligned}
\widehat{\boldsymbol{\phi}}^{+}\left(r, r^{\prime}\right) & =\frac{1}{\sqrt{2 p_{+} p_{-}}} \boldsymbol{w}^{\uparrow}\left(r, r^{\prime}\right), \\
\widehat{\boldsymbol{\phi}}^{-}\left(r, p_{-}-r^{\prime}\right) & =\frac{1}{\sqrt{2 p_{+} p_{-}}} \boldsymbol{w}^{\leftarrow}\left(r, r^{\prime}\right), \\
\widehat{\boldsymbol{\phi}}^{-}\left(p_{+}-r, r^{\prime}\right) & =\frac{1}{\sqrt{2 p_{+} p_{-}}} \boldsymbol{w}^{\rightarrow}\left(r, r^{\prime}\right), \quad\left(r, r^{\prime}\right) \in \mathcal{I}_{1}, \\
\widehat{\boldsymbol{\phi}}^{+}\left(p_{+}-r, p_{-}-r^{\prime}\right) & =\frac{1}{\sqrt{2 p_{+} p_{-}}} \boldsymbol{w}^{\downarrow}\left(r, r^{\prime}\right),
\end{aligned}
$$




$$
\begin{aligned}
& \widehat{\boldsymbol{\phi}}^{+}\left(p_{+}, r^{\prime}\right)=(-1)^{p_{-}+r^{\prime}} \frac{p_{+}}{2 p_{-}} \sqrt{\frac{p_{+} p_{-}}{2}} \boldsymbol{v}^{\uparrow}\left(p_{+}, r^{\prime}\right), \quad 1 \leqslant r^{\prime} \leqslant p_{-}-1, \\
& \widehat{\boldsymbol{\phi}}^{-}\left(p_{+}, p_{-}-r^{\prime}\right)=(-1)^{p_{-}+r^{\prime}} \frac{p_{+}}{2 p_{-}} \sqrt{\frac{p_{+} p_{-}}{2}} \boldsymbol{v}^{\leftarrow}\left(p_{+}, r^{\prime}\right), \\
& \widehat{\boldsymbol{\phi}}^{+}\left(r, p_{-}\right)=(-1)^{p_{+}+r} \frac{p_{-}}{2 p_{+}} \sqrt{\frac{p_{+} p_{-}}{2}} \boldsymbol{v}^{\uparrow}\left(r, p_{-}\right), \quad 1 \leqslant r \leqslant p_{+}-1, \\
& \widehat{\boldsymbol{\phi}}^{-}\left(p_{+}-r, p_{-}\right)=(-1)^{p_{+}+r} \frac{p_{-}}{2 p_{+}} \sqrt{\frac{p_{+} p_{-}}{2}} \boldsymbol{v}^{\rightarrow}\left(r, p_{-}\right), \quad \\
& \widehat{\boldsymbol{\phi}}^{+}\left(p_{+}, p_{-}\right)=\sqrt{2}\left(p_{+} p_{-}\right)^{3 / 2} \boldsymbol{e}\left(p_{+}, p_{-}\right), \\
& \widehat{\boldsymbol{\phi}}^{-}\left(p_{+}, p_{-}\right)=(-1)^{p_{+}+p_{-}} \sqrt{2}\left(p_{+} p_{-}\right)^{3 / 2} \boldsymbol{e}\left(0, p_{-}\right) ;
\end{aligned}
$$

(2) the Radford images of $\gamma^{\nwarrow /}\left(r, r^{\prime}\right)$ with $1 \leqslant r \leqslant p_{+}-1,1 \leqslant r^{\prime} \leqslant p_{-}$and of $\gamma^{\nwarrow \searrow}\left(r, r^{\prime}\right)$ with $1 \leqslant r \leqslant p_{+}, 1 \leqslant r^{\prime} \leqslant p_{-}-1$ are given by

$$
\begin{aligned}
& \widehat{\boldsymbol{\phi}}^{\nwarrow}\left(r, r^{\prime}\right)=(-1)^{r^{\prime}} \frac{\mathfrak{q}_{+}^{p_{-} r}+\mathfrak{q}_{+}^{-p_{-} r}}{\mathfrak{q}_{+}^{p_{-}}-\mathfrak{q}_{+}^{-p_{-}}}\left(\widehat{\boldsymbol{\phi}}^{+}\left(r, r^{\prime}\right)+\widehat{\boldsymbol{\phi}}^{-}\left(p_{+}-r, r^{\prime}\right)\right) \\
& -\frac{1}{\mathfrak{q}_{+}^{p_{-} r}-\mathfrak{q}_{+}^{-p_{-} r}} \frac{p_{+}}{2 p_{-}} \sqrt{\frac{p_{+} p_{-}}{2}} \cdot \begin{cases}\boldsymbol{v}^{\nearrow}\left(r, r^{\prime}\right), & \left(r, r^{\prime}\right) \in \mathcal{I}_{1}, \\
\boldsymbol{v}^{\swarrow}\left(p_{+}-r, p_{-}-r^{\prime}\right), & \left(p_{+}-r, p_{-}-r^{\prime}\right) \in \mathcal{I}_{1},\end{cases}
\end{aligned}
$$

$$
\widehat{\boldsymbol{\phi}}^{\mathscr{X}}\left(r, p_{-}\right)=(-1)^{r+p_{+}+1} \frac{\sqrt{2}\left(p_{+} p_{-}\right)^{3 / 2}}{\mathfrak{q}_{+}^{p_{-} r}-\mathfrak{q}_{+}^{-p_{-} r}} \boldsymbol{e}\left(r, p_{-}\right)+(-1)^{p_{-}} \frac{\mathfrak{q}_{+}^{p_{-} r}+\mathfrak{q}_{+}^{-p_{-} r}}{\mathfrak{q}_{+}^{p_{-} r}-\mathfrak{q}_{+}^{-p_{-} r}} \widehat{\boldsymbol{\varkappa}}\left(r, p_{-}\right),
$$

$$
\begin{aligned}
& \widehat{\boldsymbol{\phi}}^{\nwarrow \searrow}\left(r, r^{\prime}\right)=(-1)^{r} \frac{\mathfrak{q}_{-}^{p_{+} r^{\prime}}+\mathfrak{q}_{-}^{-p_{+} r^{\prime}}}{\mathfrak{q}_{-}^{p_{+} r^{\prime}}-\mathfrak{q}_{-}^{-p_{+} r^{\prime}}}\left(\widehat{\boldsymbol{\phi}}^{+}\left(r, r^{\prime}\right)+\widehat{\boldsymbol{\phi}}^{-}\left(r, p_{-}-r^{\prime}\right)\right) \\
& -\frac{1}{\mathfrak{q}_{-}^{p_{+} r^{\prime}}-\mathfrak{q}_{-}^{-p_{+} r^{\prime}}} \frac{p_{-}}{2 p_{+}} \sqrt{\frac{p_{+} p_{-}}{2}} \cdot \begin{cases}\boldsymbol{v}^{\nwarrow}\left(r, r^{\prime}\right), & \left(r, r^{\prime}\right) \in \mathcal{I}_{1}, \\
\boldsymbol{v}^{\searrow}\left(p_{+}-r, p_{-}-r^{\prime}\right), & \left(p_{+}-r, p_{-}-r^{\prime}\right) \in \mathcal{I}_{1},\end{cases}
\end{aligned}
$$

(3.13) $\widehat{\phi}^{\nwarrow \searrow}\left(p_{+}, r^{\prime}\right)=$

$$
=(-1)^{r^{\prime}+p_{-}+1} \frac{\sqrt{2}\left(p_{+} p_{-}\right)^{3 / 2}}{\mathfrak{q}_{-}^{p_{+} r^{\prime}}-\mathfrak{q}_{-}^{-p_{+} r^{\prime}}} \boldsymbol{e}\left(p_{+}, r^{\prime}\right)+(-1)^{p_{+}} \frac{\mathfrak{q}_{-}^{p_{+} r^{\prime}}+\mathfrak{q}_{-}^{-p_{+} r^{\prime}}}{\mathfrak{q}_{-}^{p_{+} r^{\prime}}-\mathfrak{q}_{-}^{-p_{+} r^{\prime}}} \widehat{\varkappa}\left(p_{+}, r^{\prime}\right) ;
$$

(3) and the Radford images of $\gamma^{\uparrow}\left(r, r^{\prime}\right),\left(r, r^{\prime}\right) \in \mathcal{I}_{1}$, are

$$
\begin{aligned}
& \widehat{\boldsymbol{\phi}}^{\uparrow}\left(r, r^{\prime}\right)=\frac{\sqrt{2}\left(p_{+} p_{-}\right)^{3 / 2}}{\left(\mathfrak{q}_{+}^{p_{-} r}-\mathfrak{q}_{+}^{-p_{-} r}\right)\left(\mathfrak{q}_{-}^{p_{+} r^{\prime}}-\mathfrak{q}_{-}^{-p_{+} r^{\prime}}\right)} \boldsymbol{e}\left(r, r^{\prime}\right) \\
& +(-1)^{r^{\prime}} \frac{\mathfrak{q}_{+}^{p_{-} r}+\mathfrak{q}_{+}^{-p_{-} r}}{\mathfrak{q}_{+}^{p_{-} r}-\mathfrak{q}_{+}^{-p_{-} r}}\left(\widehat{\boldsymbol{\phi}}^{\nwarrow \searrow}\left(r, r^{\prime}\right)-(-1)^{p_{+}} \widehat{\boldsymbol{\phi}}^{\nwarrow \searrow}\left(p_{+}-r, p_{-}-r^{\prime}\right)\right) \\
& +(-1)^{r} \frac{\mathfrak{q}_{-}^{p_{+} r^{\prime}}+\mathfrak{q}_{-}^{-p_{+} r^{\prime}}}{\mathfrak{q}_{-}^{p_{+} r^{\prime}}-\mathfrak{q}_{-}^{-p_{+} r^{\prime}}}\left(\widehat{\phi}^{\not{\varkappa}}\left(r, r^{\prime}\right)-(-1)^{p_{-}} \widehat{\boldsymbol{\phi}}^{\nwarrow /}\left(p_{+}-r, p_{-}-r^{\prime}\right)\right) \\
& -(-1)^{r+r^{\prime}} \frac{\left(\mathfrak{q}_{+}^{p_{-} r}+\mathfrak{q}_{+}^{-p_{-} r}\right)\left(\mathfrak{q}_{-}^{p_{+} r^{\prime}}+\mathfrak{q}_{-}^{-p_{+} r^{\prime}}\right)}{\left(\mathfrak{q}_{+}^{p_{-}}-\mathfrak{q}_{+}^{-p_{-} r}\right)\left(\mathfrak{q}_{-}^{p_{+} r^{\prime}}-\mathfrak{q}_{-}^{-p_{+} r^{\prime}}\right)} \widehat{\varkappa}\left(r, r^{\prime}\right),
\end{aligned}
$$


where we use the notation

$$
\widehat{\varkappa}\left(r, r^{\prime}\right)=\widehat{\boldsymbol{\phi}}^{+}\left(r, r^{\prime}\right)+\widehat{\boldsymbol{\phi}}^{-}\left(p_{+}-r, r^{\prime}\right)+\widehat{\boldsymbol{\phi}}^{-}\left(r, p_{-}-r^{\prime}\right)+\widehat{\boldsymbol{\phi}}^{+}\left(p_{+}-r, p_{-}-r^{\prime}\right) .
$$

The $\frac{1}{2}\left(3 p_{+}-1\right)\left(3 p_{-}-1\right)$ elements $\widehat{\phi}^{\bullet}\left(r, r^{\prime}\right)$ are a basis in Z, and therefore the $\gamma^{\bullet}\left(r, r^{\prime}\right)$ are a basis in $\mathrm{Ch}$.

This is proved in B.4.3.

3.2.2. We thus have a basis in the center $Z$ of $\mathfrak{g}_{p_{+}, p_{-}}$associated with the Radford map:

$$
\begin{aligned}
& \widehat{\boldsymbol{\phi}}^{+}\left(p_{+}, p_{-}\right), \quad \widehat{\boldsymbol{\phi}}^{\nwarrow}\left(r, p_{-}\right), \quad \widehat{\boldsymbol{\phi}}^{\uparrow}\left(r, r^{\prime}\right), \quad \widehat{\boldsymbol{\phi}}^{\nwarrow \searrow}\left(p_{+}, r^{\prime}\right), \quad \widehat{\boldsymbol{\phi}}^{-}\left(p_{+}, p_{-}\right), \\
& 1 \leqslant r \leqslant p_{+}-1 \quad\left(r, r^{\prime}\right) \in \mathcal{I}_{1} \quad 1 \leqslant r^{\prime} \leqslant p_{-}-1 \\
& \widehat{\boldsymbol{\phi}}^{+}\left(r, p_{-}\right), \widehat{\boldsymbol{\phi}}^{-}\left(p_{+}-r, p_{-}\right), \quad \widehat{\boldsymbol{\phi}}^{\nwarrow}\left(r, r^{\prime}\right), \widehat{\boldsymbol{\phi}}^{\nwarrow \searrow}\left(r, r^{\prime}\right), \quad \widehat{\boldsymbol{\phi}}^{+}\left(p_{+}, r^{\prime}\right), \widehat{\boldsymbol{\phi}}^{-}\left(p_{+}, p_{-}-r^{\prime}\right), \\
& 1 \leqslant r \leqslant p_{+}-1 \quad 1 \leqslant r \leqslant p_{+}-1, \quad 1 \leqslant r^{\prime} \leqslant p_{-}-1 \\
& 1 \leqslant r^{\prime} \leqslant p_{-}-1 \\
& \widehat{\boldsymbol{\phi}}^{+}\left(r, r^{\prime}\right), \widehat{\boldsymbol{\phi}}^{-}\left(p_{+}-r, r^{\prime}\right), \widehat{\boldsymbol{\phi}}^{-}\left(r, p_{-}-r^{\prime}\right), \widehat{\boldsymbol{\phi}}^{+}\left(p_{+}-r, p_{-}-r^{\prime}\right) \text {. } \\
& \left(r, r^{\prime}\right) \in \mathcal{I}_{1}
\end{aligned}
$$

The first line comprises $1+\left(p_{+}-1\right)+\frac{1}{2}\left(p_{+}-1\right)\left(p_{-}-1\right)+\left(p_{-}-1\right)+1=\frac{1}{2}\left(p_{+}+1\right)$. $\left(p_{-}+1\right)$ elements that are expressed as the corresponding primitive idempotents plus possible nilpotent elements. The second line comprises $2\left(p_{+}-1\right)+2\left(p_{+}-1\right)\left(p_{-}-1\right)+$ $2\left(p_{-}-1\right)$ elements in the radical, and the bottom line $4 \cdot \frac{1}{2}\left(p_{+}-1\right)\left(p_{-}-1\right)$ elements in the radical with zero pairwise products.

The elements of this basis are called the "Radford" central elements in what follows. They are a "mixing" of the canonical central elements in 2.4.1 within each subalgebra in (2.21), and are used in decompositions in what follows instead of the canonical central elements in 2.4.1.

3.2.3. More Radford-map notation. Certain linear combinations of the Radford central elements are extensively used in what follows. For the convenience of the reader, we summarize the notation for these combinations here. We define $\widehat{\varkappa}\left(r, r^{\prime}\right)$ with $\left(r, r^{\prime}\right) \in \mathcal{I}$ as

$$
\begin{array}{rlrl}
\widehat{\boldsymbol{\varkappa}}\left(r, r^{\prime}\right)= & \widehat{\boldsymbol{\phi}}^{+}\left(r, r^{\prime}\right)+\widehat{\boldsymbol{\phi}}^{-}\left(p_{+}-r, r^{\prime}\right) & \\
& +\widehat{\boldsymbol{\phi}}^{-}\left(r, p_{-}-r^{\prime}\right)+\widehat{\boldsymbol{\phi}}^{+}\left(p_{+}-r, p_{-}-r^{\prime}\right), & & \left(r, r^{\prime}\right) \in \mathcal{I}_{1}, \\
\widehat{\boldsymbol{\varkappa}}\left(r, p_{-}\right)= & \widehat{\boldsymbol{\phi}}^{+}\left(r, p_{-}\right)+\widehat{\boldsymbol{\phi}}^{-}\left(p_{+}-r, p_{-}\right), & & 1 \leqslant r \leqslant p_{+}-1, \\
\widehat{\boldsymbol{\varkappa}}\left(p_{+}, r^{\prime}\right)= & \widehat{\boldsymbol{\phi}}^{+}\left(p_{+}, r^{\prime}\right)+\widehat{\boldsymbol{\phi}}^{-}\left(p_{+}, p_{-}-r^{\prime}\right), & & 1 \leqslant r^{\prime} \leqslant p_{-}-1, \\
\widehat{\boldsymbol{\varkappa}}\left(p_{+}, p_{-}\right)= & \widehat{\boldsymbol{\phi}}^{+}\left(p_{+}, p_{-}\right), & \\
\widehat{\boldsymbol{\varkappa}}\left(0, p_{-}\right)= & \widehat{\boldsymbol{\phi}}^{-}\left(p_{+}, p_{-}\right) &
\end{array}
$$


(as we see in what follows, these form a basis in the linear span of the Drinfeld-map image of projective-module characters. We also set

$$
\begin{aligned}
\widehat{\boldsymbol{\varphi}}\left(r, r^{\prime}\right)=\left(p_{+}-r\right)\left(p_{-}-r^{\prime}\right) \widehat{\boldsymbol{\phi}}^{+}\left(r, r^{\prime}\right)-r\left(p_{-}-r^{\prime}\right) \widehat{\boldsymbol{\phi}}^{-}\left(p_{+}-r, r^{\prime}\right) & \\
& -\left(p_{+}-r\right) r^{\prime} \widehat{\boldsymbol{\phi}}^{-}\left(r, p_{-}-r^{\prime}\right)+r r^{\prime} \widehat{\boldsymbol{\phi}}^{+}\left(p_{+}-r, p_{-}-r^{\prime}\right), \quad\left(r, r^{\prime}\right) \in \mathcal{I}_{1} .
\end{aligned}
$$

Next, we define $\widehat{\rho}^{\square}\left(r, r^{\prime}\right)$ with $\left(r, r^{\prime}\right) \in \mathcal{I}_{\square}$ as

$$
\begin{aligned}
\widehat{\boldsymbol{\rho}}^{\square}\left(r, r^{\prime}\right)= & \left(p_{+}-r\right)\left(\widehat{\boldsymbol{\phi}}^{+}\left(r, r^{\prime}\right)+\widehat{\boldsymbol{\phi}}^{-}\left(r, p_{-}-r^{\prime}\right)\right) & & \\
& -r\left(\widehat{\boldsymbol{\phi}}^{-}\left(p_{+}-r, r^{\prime}\right)+\widehat{\boldsymbol{\phi}}^{+}\left(p_{+}-r, p_{-}-r^{\prime}\right)\right), & & \left(r, r^{\prime}\right) \in \mathcal{I}_{1}, \\
\widehat{\boldsymbol{\rho}}^{\square}\left(r, p_{-}\right)= & \left(p_{+}-r\right) \widehat{\boldsymbol{\phi}}^{+}\left(r, p_{-}\right)-r \widehat{\boldsymbol{\phi}}^{-}\left(p_{+}-r, p_{-}\right), & & 1 \leqslant r \leqslant p_{+}-1,
\end{aligned}
$$

and $\widehat{\boldsymbol{\rho}}^{\Xi}\left(r, r^{\prime}\right)$ with $\left(r, r^{\prime}\right) \in \mathcal{I}_{\square}$ as

$$
\begin{aligned}
& \widehat{\boldsymbol{\rho}}^{\square}\left(r, r^{\prime}\right)=\left(p_{-}-r^{\prime}\right)\left(\widehat{\boldsymbol{\phi}}^{+}\left(r, r^{\prime}\right)+\widehat{\boldsymbol{\phi}}^{-}\left(p_{+}-r, r^{\prime}\right)\right) \\
& -r^{\prime}\left(\widehat{\boldsymbol{\phi}}^{-}\left(r, p_{-}-r^{\prime}\right)+\widehat{\boldsymbol{\phi}}^{+}\left(p_{+}-r, p_{-}-r^{\prime}\right)\right), \quad\left(r, r^{\prime}\right) \in \mathcal{I}_{1}, \\
& \widehat{\boldsymbol{\rho}}^{\nabla}\left(p_{+}, r^{\prime}\right)=\left(p_{-}-r^{\prime}\right) \widehat{\boldsymbol{\phi}}^{+}\left(p_{+}, r^{\prime}\right)-r^{\prime} \widehat{\boldsymbol{\phi}}^{-}\left(p_{+}, p_{-}-r^{\prime}\right), \quad 1 \leqslant r^{\prime} \leqslant p_{-}-1 .
\end{aligned}
$$

We also define $\widehat{\varphi}^{\square}\left(r, r^{\prime}\right)$ for $\left(r, r^{\prime}\right) \in \mathcal{I}_{\square}$ as

$$
\begin{array}{rr}
\widehat{\varphi}^{\square}\left(r, r^{\prime}\right)=(-1)^{r^{\prime}} \widehat{\phi}^{\not{L}}\left(r, r^{\prime}\right)-(-1)^{p_{-}+r^{\prime}} \widehat{\boldsymbol{\phi}}^{\not{x}}\left(p_{+}-r, p_{-}-r^{\prime}\right),\left(r, r^{\prime}\right) \in \mathcal{I}_{1}, \\
\widehat{\varphi}^{\square}\left(r, p_{-}\right)=(-1)^{p_{-}} \widehat{\boldsymbol{\phi}}^{\not{\alpha}}\left(r, p_{-}\right), & 1 \leqslant r \leqslant p_{+}-1,
\end{array}
$$

and $\widehat{\varphi}^{\nabla}\left(r, r^{\prime}\right)$ for $\left(r, r^{\prime}\right) \in \mathcal{I}_{\square}$ as

$$
\begin{aligned}
\widehat{\varphi}^{\square}\left(r, r^{\prime}\right) & =(-1)^{r} \widehat{\boldsymbol{\phi}}^{\nwarrow \searrow}\left(r, r^{\prime}\right)-(-1)^{p_{+}+r} \widehat{\boldsymbol{\phi}}^{\nwarrow \searrow}\left(p_{+}-r, p_{-}-r^{\prime}\right), & & \left(r, r^{\prime}\right) \in \mathcal{I}_{1}, \\
\widehat{\varphi}^{\square}\left(p_{+}, r^{\prime}\right) & =(-1)^{p_{+}} \widehat{\boldsymbol{\phi}}^{\nwarrow \searrow}\left(p_{+}, r^{\prime}\right), & & 1 \leqslant r^{\prime} \leqslant p_{-}-1 .
\end{aligned}
$$

\section{THE FACTORIZABLE AND RIBBON STRUCTURES FOR $\mathfrak{g}_{p_{+}, p_{-}}$}

In this section, we study the Drinfeld map and a ribbon structure for $\mathfrak{g}_{p_{+}, p_{-}}$. We find the necessary ingredient for the Drinfeld map (the $M$-matrix) in 4.1. As a preparation for the study of the $S L(2, \mathbb{Z})$-action, we then evaluate the Drinfeld images of the $\gamma$ basis in Ch. Together with the "Radford" basis constructed in Sec. 3, this gives two bases in the center that play a crucial role in the study of the modular group action in Sec. 5 .

We consider the Drinfeld map in 4.2 and find a ribbon element in 4.3 . As a short detour in 4.2.6. we characterise the Grothendieck ring in terms of Chebyshev polynomials.

4.1. $M$-matrix. For a quasitriangular Hopf algebra $A$ with the universal $R$-matrix $R$, the $M$-matrix is defined as

$$
M=R_{21} R_{12} \in A \otimes A .
$$

It satisfies the relations $(\Delta \otimes \mathrm{id})(M)=R_{32} M_{13} R_{23}$ and $M \Delta(x)=\Delta(x) M \forall x \in A$. If 
in addition $M$ can be represented as

$$
M=\sum_{I} \boldsymbol{m}_{I} \otimes \boldsymbol{n}_{I}
$$

where $\boldsymbol{m}_{I}$ and $\boldsymbol{n}_{I}$ are two bases in $A$, the Hopf algebra $A$ is called factorizable.

For a Hopf algebra $A$ with an $M$-matrix, the Drinfeld map $\chi: A^{*} \rightarrow A$ is defined by

$$
\chi(\beta)=(\beta \otimes \mathrm{id}) M
$$

In a factorizable Hopf algebra $A$, the restriction of the Drinfeld map to the space $\mathrm{Ch}$ of $q$-characters gives an isomorphism $\mathrm{Ch}(A) \stackrel{\simeq}{\rightarrow} \mathrm{Z}(A)$ of associative algebras [21].

4.1.1. The $M$-matrix for $\mathfrak{g}_{p_{+}, p_{-}}$. As any Drinfeld double, the double $D(\mathcal{H})$ whose construction was outlined in 2.1 (see [22] for the detailed calculation) is endowed with a universal $R$-matrix. To evaluate it explicitly, we construct the elements of the basis dual to the PBW basis in $\mathcal{H}$, Eqs. (2.2), in terms of the generators introduced in (2.3) and thus find

$$
\begin{aligned}
& R=\frac{1}{4 p_{+} p_{-}} \sum_{m=0}^{p_{+}-1} \sum_{n=0}^{p_{-}-1} \sum_{j=0}^{4 p_{+} p_{-}-1} \sum_{c=0}^{4 p_{+} p_{-}^{-1}} \frac{(-1)^{m+n}\left(\mathfrak{q}_{+}^{p_{-}}-\mathfrak{q}_{+}^{-p_{-}}\right)^{m}\left(\mathfrak{q}_{-}^{p_{+}}-\mathfrak{q}_{-}^{-p_{+}}\right)^{n}}{[m]_{+} ![n]_{-} !} \\
& \times \mathfrak{q}_{+}^{-m j-p_{-} \frac{m(m-1)}{2}} \mathfrak{q}_{-}^{n j-p_{+} \frac{n(n-1)}{2}} \mathfrak{q}^{-c j} k^{j} e_{+}^{m} f_{-}^{n} \otimes f_{+}^{m} e_{-}^{n} \kappa^{c} .
\end{aligned}
$$

The $M$-matrix for $D(\mathcal{H})$ is $M=R_{21} R_{12}$ with this $R$. By simple calculation, it then follows that the $M$-matrix $\bar{M}$ for $\bar{D}(\mathcal{H})$ (see (2.4) $)$ is given by

$$
\begin{aligned}
& \bar{M}=\frac{1}{2 p_{+} p_{-}} \sum_{j^{\prime}=0}^{2 p_{+} p_{-}} \sum_{j=0}^{-1} \sum_{m=0}^{2 p_{+} p_{-}-1} \sum_{n=0}^{p_{+}-1} \sum_{m^{\prime}=0}^{p_{p_{+}}^{-1}} \sum_{n^{\prime}=0}^{p_{-}^{-1}} \frac{\left(\mathfrak{q}_{+}^{-p_{-}}-\mathfrak{q}_{+}^{p_{-}}\right)^{m+n}\left(\mathfrak{q}_{-}^{-p_{+}}-\mathfrak{q}_{-}^{p_{+}}\right)^{m^{\prime}+n^{\prime}}}{[m]_{+} !\left[m^{\prime}\right]_{-} ![n]_{+} !\left[n^{\prime}\right]_{-} !}
\end{aligned}
$$

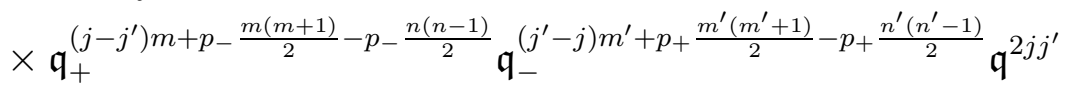

$$
\begin{aligned}
& \times f_{+}^{n} e_{+}^{m} e_{-}^{n^{\prime}} f_{-}^{m^{\prime}} k^{2 j} \otimes e_{+}^{n} f_{+}^{m} f_{-}^{n^{\prime}} e_{-}^{m^{\prime}} k^{2 j^{\prime}},
\end{aligned}
$$

which involves only even powers of $k$, and therefore $\bar{M} \in \mathfrak{g}_{p_{+}, p_{-}} \otimes \mathfrak{g}_{p_{+}, p_{-}}$. To repeat this in words, the attempted calculation of the $M$-matrix for $\bar{D}(\mathcal{H})$ has yielded the $M$-matrix for its $\mathfrak{g}_{p_{+}, p_{-}}$subalgerba (and hence $\bar{D}(\mathcal{H})$ is not a factorizable quantum group; as noted in the introduction, the relevant object is the pair $\mathfrak{g}_{p_{+}, p} \subset \bar{D}(\mathcal{H})$ of quantum groups one of which has an $M$-matrix and the other an $R$-matrix). 7 We next use $\bar{M}$ to construct the Drinfeld map for $\mathfrak{g}_{p_{+}, p_{-}}$.

\subsection{The Drinfeld map for $\mathfrak{g}_{p_{+}, p_{-}}$.}

4.2.1. Drinfeld map of the Grothendieck ring. In considering the Drinfeld map, we

\footnotetext{
${ }^{7}$ This is rather natural from the conformal field theory standpoint: the $R$-matrix acting on two vertex operators describes their braiding, while the $M$-matrix describes its "square," the operation of taking one of the operators around the other. The braiding requires choosing one of the two possible directions, which corresponds to choosing how $k=\sqrt{K}$ acts on the representations.
} 
first restrict it to the image of the Grothendieck ring in the space Ch of $q$-characters under the map $\mathrm{X} \mapsto \mathrm{qCh}$ (

$$
\chi \circ \mathrm{qCh}: \mathrm{G}(A) \rightarrow \mathrm{Z}(A),
$$

which is a homomorphism of associative commutative algebras for any factorizable ribbon Hopf algebra $A$.

With the balancing element for $A=\mathfrak{g}_{p_{+}, p_{-}}$given by (3.6), Eq. (4.4) becomes

$$
\begin{aligned}
\mathrm{G}_{2 p_{+} p_{-}} & \rightarrow \mathrm{Z} \\
\mathrm{X}_{r, r^{\prime}}^{\alpha} & \mapsto \chi^{\alpha}\left(r, r^{\prime}\right) \equiv \chi\left(\gamma^{\alpha}\left(r, r^{\prime}\right)\right)=\left(\operatorname{Tr}_{\mathrm{X}_{r, r^{\prime}}^{\alpha}} \otimes \mathrm{id}\right)\left(\left(K^{p_{-}-p_{+}} \otimes \mathbf{1}\right) \bar{M}\right),
\end{aligned}
$$

where $1 \leqslant r \leqslant p_{+}, 1 \leqslant r^{\prime} \leqslant p_{-}$, and $\alpha= \pm$. Clearly, $\chi^{+}(1,1)=1$. The other $\chi^{ \pm}\left(r, r^{\prime}\right)$ are evaluated as follows.

4.2.2. Proposition. For $1 \leqslant r \leqslant p_{+}$and $1 \leqslant r^{\prime} \leqslant p_{-}$,

$$
\begin{aligned}
& \chi^{+}\left(r, r^{\prime}\right)=\chi_{(+)}(r) \chi_{(-)}\left(r^{\prime}\right), \\
& \chi^{-}\left(r, r^{\prime}\right)=(-1)^{p_{-}+p_{+}} \chi^{+}\left(r, r^{\prime}\right) K^{p_{+} p_{-}},
\end{aligned}
$$

where

$$
\begin{aligned}
\chi_{( \pm)}(r)=(-1)^{r-1} \sum_{a=0}^{r-1} \sum_{m=0}^{a}\left(\mathfrak{q}_{ \pm}^{p_{\mp}}-\mathfrak{q}_{ \pm}^{-p_{\mp}}\right)^{2 m} \mathfrak{q}_{ \pm}^{p_{\mp} m(m+r-2 a)+p_{\mp}(r-1-2 a)} \\
\times\left[\begin{array}{c}
r-a+m-1 \\
m
\end{array}\right]_{ \pm}\left[\begin{array}{c}
a \\
m
\end{array}\right]_{ \pm} e_{ \pm}^{m} f_{ \pm}^{m} K_{ \pm}^{-m-r+1+2 a}
\end{aligned}
$$

Moreover, for $1 \leqslant r \leqslant p_{+}, 1 \leqslant r^{\prime} \leqslant p_{-}$, and $\alpha= \pm, \quad \chi^{\alpha}\left(r, r^{\prime}\right)$ decomposes in terms of the Radford central elements as (recall the notation in (3.16)-(3.29)

$$
\begin{aligned}
& \chi^{\alpha}\left(r, r^{\prime}\right)=\frac{r r^{\prime}}{\sqrt{2}\left(p_{+} p_{-}\right)^{3 / 2}} \widehat{\varkappa}\left(p_{+}, p_{-}\right)+(-1)^{r p_{-}+r^{\prime} p_{+}+\beta p_{+} p_{-}} \frac{r r^{\prime}}{\sqrt{2}\left(p_{+} p_{-}\right)^{3 / 2}} \widehat{\varkappa}\left(0, p_{-}\right) \\
& -r^{\prime} \sum_{s=1}^{p_{+}-1}(-1)^{(r-1) p_{-}+\left(\beta p_{-}+r^{\prime}\right)\left(s+p_{+}\right)} \frac{\mathfrak{q}_{+}^{p_{-} r s}-\mathfrak{q}_{+}^{-p_{-} r s}}{\sqrt{2}\left(p_{+} p_{-}\right)^{3 / 2}} \widehat{\phi}^{\not{ }}\left(s, p_{-}\right) \\
& +r r^{\prime} \sum_{s=1}^{p_{+}-1}(-1)^{r p_{-}+\left(\beta p_{--} r^{\prime}\right)\left(p_{+}-s\right)} \frac{\mathfrak{q}_{+}^{p_{-} r s}+\mathfrak{q}_{+}^{-p_{-} r s}}{\sqrt{2}\left(p_{+} p_{-}\right)^{3 / 2}} \widehat{\varkappa}\left(s, p_{-}\right) \\
& -r \sum_{s^{\prime}=1}^{p_{-}-1}(-1)^{\left(\beta p_{+}+r\right)\left(s^{\prime}+p_{-}\right)+\left(r^{\prime}-1\right) p_{+}} \frac{\mathfrak{q}_{-}^{p_{+} r^{\prime} s^{\prime}}-\mathfrak{q}_{-}^{-p_{+} r^{\prime} s^{\prime}}}{\sqrt{2}\left(p_{+} p_{-}\right)^{3 / 2}} \widehat{\phi}^{\nwarrow \searrow}\left(p_{+}, s^{\prime}\right) \\
& +r r^{\prime} \sum_{s^{\prime}=1}^{p_{-}-1}(-1)^{r^{\prime} p_{+}+\left(\beta p_{+}-r\right)\left(p_{-}-s^{\prime}\right)} \frac{\mathfrak{q}_{-}^{p_{+} r^{\prime} s^{\prime}}+\mathfrak{q}_{-}^{-p_{+} r^{\prime} s^{\prime}}}{\sqrt{2}\left(p_{+} p_{-}\right)^{3 / 2}} \widehat{\boldsymbol{\varkappa}}\left(p_{+}, s^{\prime}\right) \\
& +\sum_{\left(s, s^{\prime}\right) \in \mathcal{I}_{1}}(-1)^{\left(\beta p_{+}+r-1\right) s^{\prime}+\left(\beta p_{-}+r^{\prime}-1\right) s} \frac{\left(\mathfrak{q}_{+}^{p_{-} r s}-\mathfrak{q}_{+}^{-p_{-} r s}\right)\left(\mathfrak{q}_{-}^{p_{+} r^{\prime} s^{\prime}}-\mathfrak{q}_{-}^{-p_{+} r^{\prime} s^{\prime}}\right)}{\sqrt{2}\left(p_{+} p_{-}\right)^{3 / 2}} \widehat{\boldsymbol{\phi}}^{\uparrow}\left(s, s^{\prime}\right)
\end{aligned}
$$




$$
\begin{gathered}
-\sum_{\left(s, s^{\prime}\right) \in \mathcal{I}_{1}}(-1)^{\left(\beta p_{+}-r\right) s^{\prime}+\left(\beta p_{-}-r^{\prime}\right) s}\left(r^{\prime} \frac{\left(\mathfrak{q}_{-}^{p_{+} r^{\prime} s^{\prime}}+\mathfrak{q}_{-}^{-p_{+} r^{\prime} s^{\prime}}\right)\left(\mathfrak{q}_{+}^{p_{-} r s}-\mathfrak{q}_{+}^{-p_{-} r s}\right)}{\sqrt{2}\left(p_{+} p_{-}\right)^{3 / 2}} \widehat{\varphi}^{\square}\left(s, s^{\prime}\right)\right. \\
\left.+r \frac{\left(\mathfrak{q}_{+}^{p_{-} r s}+\mathfrak{q}_{+}^{-p_{-} r s}\right)\left(\mathfrak{q}_{-}^{p_{+} r^{\prime} s^{\prime}}-\mathfrak{q}_{-}^{-p_{+} r^{\prime} s^{\prime}}\right)}{\sqrt{2}\left(p_{+} p_{-}\right)^{3 / 2}} \widehat{\varphi}^{\square}\left(s, s^{\prime}\right)\right) \\
+r r^{\prime} \sum_{\left(s, s^{\prime}\right) \in \mathcal{I}_{1}}(-1)^{\left(\beta p_{+}-r\right) s^{\prime}+\left(\beta p_{-} r^{\prime}\right) s} \frac{\left(\mathfrak{q}_{+}^{p_{-} r s}+\mathfrak{q}_{+}^{-p_{-} r s}\right)\left(\mathfrak{q}_{-}^{p_{+} r^{\prime} s^{\prime}}+\mathfrak{q}_{-}^{-p_{-} r^{\prime} s^{\prime}}\right)}{\sqrt{2}\left(p_{+} p_{-}\right)^{3 / 2}} \widehat{\varkappa}\left(s, s^{\prime}\right),
\end{gathered}
$$

where $\alpha=(-1)^{\beta}$ (that is, $\beta=0$ for $\chi^{+}\left(r, r^{\prime}\right)$ and $\beta=1$ for $\chi^{-}\left(r, r^{\prime}\right)$ ).

Because (4.4) is an algebra homomorphism, the product $\chi^{\alpha}\left(r, r^{\prime}\right) \chi^{\alpha^{\prime}}\left(s, s^{\prime}\right)$ is expressed through the $\chi^{ \pm}\left(t, t^{\prime}\right)$ just by the formulas in 1.2 with $\mathrm{X}^{ \pm} \rightarrow \chi^{ \pm}$and $\widetilde{\mathrm{X}}^{ \pm} \rightarrow \widetilde{\chi}^{ \pm}$(cf. [5]).

The proof of (4.6) $-(4.7)$ is by straightforward calculation based on (B.10) and (B.13). Using (2.6), we then find that

$$
\begin{aligned}
\operatorname{Tr}_{\mathbf{X}^{\alpha}\left(r, r^{\prime}\right)}\left(f_{+}^{m} e_{+}^{m} e_{-}^{m^{\prime}} f_{-}^{m^{\prime}} K^{j+p_{-}-p_{+}}\right)= & \\
=\sum_{a=m}^{r-1} & \sum_{a^{\prime}=0}^{r^{\prime}-1-m^{\prime}} \alpha^{m p_{-}+m^{\prime} p_{+}+j+p_{-}-p_{+}}(-1)^{m\left(r^{\prime}-1\right)+m^{\prime}(r-1)+r+r^{\prime}}\left([m]_{+} !\left[m^{\prime}\right]_{-} !\right)^{2} \\
& \times\left[\begin{array}{c}
r-a+m-1 \\
m
\end{array}\right]_{+}\left[\begin{array}{c}
a \\
m
\end{array}\right]_{+}\left[\begin{array}{c}
r^{\prime}-a^{\prime}-1 \\
m^{\prime}
\end{array}\right]_{-}\left[\begin{array}{c}
a^{\prime}+m^{\prime} \\
m^{\prime}
\end{array}\right]_{-} \mathfrak{q}_{+}^{(r-1-2 a)\left(j+p_{-}\right)} \mathfrak{q}_{-}^{\left(r^{\prime}-1-2 a^{\prime}\right)\left(j-p_{+}\right)},
\end{aligned}
$$

which after some rearrangements gives (4.6)-(4.7) in accordance with (4.5) for the $M$ matrix in (4.3). The decomposition then follows by calculating the action on projective modules, as explained in B.2.2.

4.2.3. Remark. Unlike the $M$-matrix, the Drinfeld map in (4.6) factors into a product of the Drinfeld maps (4.7) for the two $\overline{\mathcal{U}}_{Q_{ \pm}} s \ell(2)$ quantum groups with $Q_{ \pm}=\mathfrak{q}_{ \pm}^{p_{\mp}}$. The corresponding multiplication formulas are known from [2, 5]:

where

$$
\boldsymbol{\chi}_{( \pm)}(r) \boldsymbol{\chi}_{( \pm)}\left(r^{\prime}\right)=\sum_{\substack{r^{\prime \prime}=\left|r-r^{\prime}\right|+1 \\ \text { step }=2}}^{r+r^{\prime}-1} \widetilde{\boldsymbol{\chi}}_{( \pm)}\left(r^{\prime \prime}\right)
$$

$$
\widetilde{\boldsymbol{\chi}}_{( \pm)}(r)= \begin{cases}\chi_{( \pm)}(r), & 1 \leqslant r \leqslant p_{ \pm} \\ \chi_{( \pm)}\left(2 p_{ \pm}-r\right)+2(-1)^{p_{-}+p_{+}} \chi_{( \pm)}\left(r-p_{ \pm}\right) K_{ \pm}^{p_{ \pm}}, & p_{ \pm}+1 \leqslant r \leqslant 2 p_{ \pm}-1\end{cases}
$$

We recall that $K_{+}^{p_{+}}=K_{-}^{p_{-}}=K^{p_{+} p_{-}}$in $\mathfrak{g}_{p_{+}, p_{-}}$.

Naturally, relations (4.9) become the corresponding formulas in [5] in the cases where either $p_{+}=1$ or $p_{-}=1$.

4.2.4. Drinfeld images of pseudotraces. We next evaluate the Drinfeld map on the $\gamma^{\pi / x}$, $\gamma^{\star \searrow}$, and $\gamma^{\uparrow}$ in (2.20). This gives

$$
\chi^{\nwarrow \searrow}\left(r, r^{\prime}\right)=\chi\left(\gamma^{\not{x}}\left(r, r^{\prime}\right)\right)=
$$




$$
=(-1)^{r^{\prime}}\left(\operatorname{Tr}_{\mathbb{P}_{r, r^{\prime}}} \otimes \mathrm{id}\right)\left(\left(K^{p_{-}-p_{+}} \otimes \mathbf{1}\right) \bar{M}\left(\sigma^{\not{\not}}\left(r, r^{\prime}\right) \otimes \mathrm{id}\right)\right)
$$

(4.11) $\chi^{\not{\not}}\left(r, r^{\prime}\right)=\chi\left(\gamma^{\nwarrow \searrow}\left(r, r^{\prime}\right)\right)$

$$
=(-1)^{r}\left(\operatorname{Tr}_{\mathbb{P}_{r, r^{\prime}}} \otimes \mathrm{id}\right)\left(\left(K^{p_{-}-p_{+}} \otimes \mathbf{1}\right) \bar{M}\left(\sigma^{\nwarrow \searrow}\left(r, r^{\prime}\right) \otimes \mathrm{id}\right)\right)
$$

for all $1 \leqslant r \leqslant p_{+}-1,1 \leqslant r^{\prime} \leqslant p_{-}-1$, and

(4.12) $\quad \chi^{\Downarrow}\left(r, r^{\prime}\right)=\chi\left(\gamma^{\uparrow}\left(r, r^{\prime}\right)\right)$

$$
=(-1)^{r+r^{\prime}}\left(\operatorname{Tr}_{\mathbb{P}_{r, r^{\prime}}} \otimes \mathrm{id}\right)\left(\left(K^{p_{-}-p_{+}} \otimes \mathbf{1}\right) \bar{M}\left(\sigma^{\Uparrow}\left(r, r^{\prime}\right) \otimes \mathrm{id}\right)\right)
$$

for $\left(r, r^{\prime}\right) \in \mathcal{I}_{1}$.

4.2.5. Proposition. The central elements $\chi^{\nwarrow \searrow}\left(r, r^{\prime}\right), \chi^{\not{\not}}\left(r, r^{\prime}\right)$, and $\chi^{\downarrow}\left(r, r^{\prime}\right)$ decompose in terms of the Radford central elements as

$$
\begin{aligned}
(-1)^{r^{\prime}} \chi^{\nwarrow \searrow}\left(r, r^{\prime}\right) & =r^{\prime} \sum_{s=1}^{p_{+}-1}(-1)^{r^{\prime}\left(s+p_{+}\right)+p_{-} r} \frac{\mathfrak{q}_{+}^{p_{-} r s}-\mathfrak{q}_{+}^{-p_{-} r s}}{p_{-} \sqrt{2 p_{+} p_{-}}} \widehat{\boldsymbol{\rho}}^{\square}\left(s, p_{-}\right) \\
+ & r^{\prime} \sum_{\left(s, s^{\prime}\right) \in \mathcal{I}_{1}}(-1)^{r s^{\prime}+r^{\prime} s} \frac{\left(\mathfrak{q}_{+}^{p_{-} r s}-\mathfrak{q}_{+}^{-p_{-} r s}\right)\left(\mathfrak{q}_{-}^{p_{+} r^{\prime} s^{\prime}}+\mathfrak{q}_{-}^{-p_{+} r^{\prime} s^{\prime}}\right)}{p_{-} \sqrt{2 p_{+} p_{-}}} \widehat{\boldsymbol{\rho}}^{\square}\left(s, s^{\prime}\right) \\
& -\sum_{\left(s, s^{\prime}\right) \in \mathcal{I}_{1}}(-1)^{r s^{\prime}+r^{\prime} s} \frac{\left(\mathfrak{q}_{+}^{p_{-} r s}-\mathfrak{q}_{+}^{-p_{-} r s}\right)\left(\mathfrak{q}_{-}^{p_{+} r^{\prime} s^{\prime}}-\mathfrak{q}_{-}^{-p_{+} r^{\prime} s^{\prime}}\right)}{p_{-} \sqrt{2 p_{+} p_{-}}} \widehat{\varphi}^{\nwarrow \searrow}\left(s, s^{\prime}\right),
\end{aligned}
$$

$$
\begin{aligned}
(-1)^{p_{-}} \boldsymbol{\chi}^{\nwarrow \searrow}\left(r, p_{-}\right)=\frac{\mathfrak{q}_{+}^{p_{-}-\mathfrak{q}_{+}^{-p_{-}}}}{\sqrt{2 p_{+} p_{-}}}\left(\sum_{s=1}^{p_{+}-1}(-1)^{p_{-}\left(s+p_{+}+r\right)}[r s]_{+} \widehat{\boldsymbol{\rho}}^{\square}\left(s, p_{-}\right)\right. \\
\left.\quad+2 \sum_{\left(s, s^{\prime}\right) \in \mathcal{I}_{1}}(-1)^{\left(r+p_{+}\right) s^{\prime}+p_{-} s}[r s]_{+} \widehat{\boldsymbol{\rho}}^{\square}\left(s, s^{\prime}\right)\right),
\end{aligned}
$$

$$
\begin{aligned}
& (-1)^{r} \chi^{\not{\alpha}}\left(r, r^{\prime}\right)=r \sum_{s^{\prime}=1}^{p_{-}-1}(-1)^{r\left(s^{\prime}+p_{-}\right)+p_{+} r^{\prime}} \frac{\mathfrak{q}_{-}^{p_{+} r^{\prime} s^{\prime}}-\mathfrak{q}_{-}^{-p_{+} r^{\prime} s^{\prime}}}{p_{+} \sqrt{2 p_{+} p_{-}}} \widehat{\boldsymbol{\rho}}^{\nabla}\left(p_{+}, s^{\prime}\right) \\
& +r \sum_{\left(s, s^{\prime}\right) \in \mathcal{I}_{1}}(-1)^{r^{\prime} s+r s^{\prime}} \frac{\left(\mathfrak{q}_{+}^{p_{-} r s}+\mathfrak{q}_{+}^{-p_{-} r s}\right)\left(\mathfrak{q}_{-}^{p_{+} r^{\prime} s^{\prime}}-\mathfrak{q}_{-}^{-p_{+} r^{\prime} s^{\prime}}\right)}{p_{+} \sqrt{2 p_{+} p_{-}}} \widehat{\boldsymbol{\rho}}^{\nabla}\left(s, s^{\prime}\right) \\
& -\sum_{\left(s, s^{\prime}\right) \in \mathcal{I}_{1}}(-1)^{r^{\prime} s+r s^{\prime}} \frac{\left(\mathfrak{q}_{+}^{p_{-} r s}-\mathfrak{q}_{+}^{-p_{-} r s}\right)\left(\mathfrak{q}_{-}^{p_{+} r^{\prime} s^{\prime}}-\mathfrak{q}_{-}^{-p_{+} r^{\prime} s^{\prime}}\right)}{p_{+} \sqrt{2 p_{+} p_{-}}} \widehat{\varphi}^{\not{x}}\left(s, s^{\prime}\right),
\end{aligned}
$$

$$
\begin{aligned}
(-1)^{p_{+}} \boldsymbol{\chi}^{\nwarrow /}\left(p_{+}, r^{\prime}\right)=\frac{\mathfrak{q}_{-}^{p_{+}-\mathfrak{q}_{-}^{-p_{+}}}}{\sqrt{2 p_{+} p_{-}}}\left(\sum_{s^{\prime}=1}^{p_{-}-1}(-1)^{p_{+}\left(s^{\prime}+p_{-}+r^{\prime}\right)}\left[r^{\prime} s^{\prime}\right]_{-} \widehat{\boldsymbol{\rho}}^{\square}\left(p_{+}, s^{\prime}\right)\right. \\
\left.+2 \sum_{\left(s, s^{\prime}\right) \in \mathcal{I}_{1}}(-1)^{\left(r^{\prime}+p_{-}\right) s+p_{+} s^{\prime}}\left[r^{\prime} s^{\prime}\right]_{-} \widehat{\boldsymbol{\rho}}^{\square}\left(s, s^{\prime}\right)\right),
\end{aligned}
$$




$$
(-1)^{r+r^{\prime}} \chi^{\Downarrow}\left(r, r^{\prime}\right)=\sum_{\left(s, s^{\prime}\right) \in \mathcal{I}_{1}}(-1)^{r s^{\prime}+r^{\prime} s} \frac{\left(\mathfrak{q}_{+}^{p_{-} r s}-\mathfrak{q}_{+}^{-p_{-} r s}\right)\left(\mathfrak{q}_{-}^{p_{+} r^{\prime} s^{\prime}}-\mathfrak{q}_{-}^{-p_{+} r^{\prime} s^{\prime}}\right)}{\sqrt{2 p_{+} p_{-}}} \widehat{\varphi}\left(s, s^{\prime}\right),
$$

where we use the notation in $\mathbf{3 . 2 . 3}$ and set

$$
\begin{aligned}
& \widehat{\varphi}^{\nwarrow \searrow}\left(r, r^{\prime}\right)=(-1)^{r}\left(p_{+}-r\right) \widehat{\boldsymbol{\phi}}^{\nwarrow \searrow}\left(r, r^{\prime}\right)+(-1)^{p_{+}+r} r \widehat{\boldsymbol{\phi}}^{\nwarrow \searrow}\left(p_{+}-r, p_{-}-r^{\prime}\right), \\
& \widehat{\varphi}^{\mathscr{x}}\left(r, r^{\prime}\right)=(-1)^{r^{\prime}}\left(p_{-}-r^{\prime}\right) \widehat{\boldsymbol{\phi}}^{\mathscr{L}}\left(r, r^{\prime}\right)+(-1)^{p_{-}+r^{\prime}} r^{\prime} \widehat{\boldsymbol{\phi}}^{\mathscr{x}}\left(p_{+}-r, p_{-}-r^{\prime}\right) \text {, }
\end{aligned}
$$

for $\left(r, r^{\prime}\right) \in \mathcal{I}_{1}$.

Proof. A calculation similar to the one in the proof of 4.2.2 shows that the $\chi^{\nwarrow \searrow}\left(r, r^{\prime}\right)$, $\chi^{\check{L}}\left(r, r^{\prime}\right)$, and $\chi^{\downarrow}\left(r, r^{\prime}\right)$ are explicitly given by

$$
\chi^{\nwarrow \searrow}\left(r, r^{\prime}\right)=(-1)^{r^{\prime}}\left(\boldsymbol{\vartheta}_{(+)}(r)-(-1)^{p_{+}+p_{-}} \boldsymbol{\vartheta}_{(+)}\left(p_{+}-r\right) K^{p_{+} p_{-}}\right) \chi_{(-)}\left(r^{\prime}\right)
$$

for $1 \leqslant r \leqslant p_{+}-1$ and $1 \leqslant r^{\prime} \leqslant p_{-}$,

$$
\boldsymbol{\chi}^{\not{\varkappa}}\left(r, r^{\prime}\right)=(-1)^{r} \boldsymbol{\chi}_{(+)}(r)\left(\boldsymbol{\vartheta}_{(-)}\left(r^{\prime}\right)-(-1)^{p_{+}+p_{-}} \boldsymbol{\vartheta}_{(-)}\left(p_{-}-r^{\prime}\right) K^{p_{+} p_{-}}\right),
$$

for $1 \leqslant r \leqslant p_{+}$and $1 \leqslant r^{\prime} \leqslant p_{-}-1$, and

$$
\begin{array}{r}
\chi^{\Downarrow}\left(r, r^{\prime}\right)=(-1)^{r+r^{\prime}}\left(\boldsymbol{\vartheta}_{(+)}(r)-(-1)^{p_{+}+p_{-}} \boldsymbol{\vartheta}_{(+)}\left(p_{+}-r\right) K^{p_{+} p_{-}}\right) \\
\times\left(\boldsymbol{\vartheta}_{(-)}\left(r^{\prime}\right)-(-1)^{p_{+}+p_{-}} \boldsymbol{\vartheta}_{(-)}\left(p_{-}-r^{\prime}\right) K^{p_{+} p_{-}}\right),
\end{array}
$$

for $\left(r, r^{\prime}\right) \in \mathcal{I}_{1}$, where $\chi_{( \pm)}$are defined in (4.7) and

$$
\begin{aligned}
\boldsymbol{\vartheta}_{( \pm)}(r)=(-1)^{r}[r]_{ \pm} \sum_{a=0}^{r-1} \sum_{m=0}^{p_{ \pm}-1} \frac{\left(\mathfrak{q}_{ \pm}^{p_{\mp}}-\mathfrak{q}_{ \pm}^{-p_{\mp}}\right)^{2 m-1}}{\left([m]_{ \pm} !\right)^{2}} & \mathfrak{q}_{ \pm}^{p_{\mp} m(m+r-2 a)+p_{\mp}(r-1-2 a)} \\
& \times\left[x^{1}\right]\left(\mathrm{C}_{ \pm, r, a}^{m}(x)\right) e_{ \pm}^{m} f_{ \pm}^{m} K^{-p_{\mp}(m+r-1-2 a)},
\end{aligned}
$$

where the polynomials $\mathrm{C}_{ \pm, r, a}^{m}(x)$ are defined in $(\underline{\mathrm{B} .18})$.

The rest is shown by evaluation of the action of $\chi^{\nwarrow \downarrow}, \chi^{\not{L}}$, and $\chi^{\downarrow}$ (that is, of $\boldsymbol{\vartheta}_{( \pm)}(r)$ and $\chi_{(+)}(r)$ ) on projective modules, see B.2.2. Because of the Drinfeld map factorization (cf. 4.2.3), the demonstration largely reduces to separate calculations in each of the two $\overline{\mathcal{U}}_{Q_{ \pm}} s \ell(2)$ quantum groups with $Q_{ \pm}=\mathfrak{q}_{ \pm}^{p_{\mp}}$, cf. [5].

4.2.6. Grothendieck ring as a polynomial quotient ring. We next characterize the Grothendieck ring as follows. Let

$$
U_{s}(2 \cos t)=\frac{\sin s t}{\sin t}, \quad s \geqslant 1,
$$

be the Chebyshev polynomials of the second kind. They satisfy (and are determined by) the recursive relation

$$
x U_{s}(x)=U_{s-1}(x)+U_{s+1}(x), \quad s \geqslant 2,
$$

with the initial data $U_{1}(x)=1, U_{2}(x)=x$. 
4.2.7. Proposition. The Grothendieck ring $\mathrm{G}_{2 p_{+} p_{-}}$of $\mathfrak{g}_{p_{+}, p_{-}}$is the quotient of $\mathbb{C}[x, y]$ over the ideal generated by the polynomials

$$
\begin{aligned}
& U_{2 p_{+}+1}(x)-U_{2 p_{+}-1}(x)-2, \\
& U_{2 p_{-}+1}(y)-U_{2 p_{-}-1}(y)-2, \\
& U_{p_{+}+1}(x)-U_{p_{+-1}}(x)-U_{p_{-+1}}(y)+U_{p_{--1}}(y) .
\end{aligned}
$$

Moreover, the basis of irreducible representations $\mathrm{X}_{r, r^{\prime}}^{ \pm}$is given by (the image under the quotient mapping of ) the respective polynomials $P_{r, r^{\prime}}^{ \pm}, 1 \leqslant r \leqslant p_{+}, 1 \leqslant r^{\prime} \leqslant p_{-}$, where

$$
\begin{aligned}
& P_{r, r^{\prime}}^{+}(x, y)=U_{r}(x) U_{r^{\prime}}(y), \\
& P_{r, r^{\prime}}^{-}(x, y)=\left(\frac{1}{2} U_{p_{+}+r}(x)-\frac{1}{2} U_{p_{+}-r}(x)\right) U_{r^{\prime}}(y) .
\end{aligned}
$$

To show 4.2.7 we first of all recall, once again, that the Drinfeld map $\chi$ is an algebra isomorphism. Next, it follows from (1.5) that the Grothendieck ring is generated by the elements

$$
\chi^{+}(2,1)=\boldsymbol{C}_{+}, \quad \chi^{+}(1,2)=\boldsymbol{C}_{-}
$$

(see $(\underline{B .11})$ and $(\overline{B .14})$, which, moreover, satisfy Eqs. (B.16), where we can rewrite $\psi_{ \pm}$ as

$$
\begin{aligned}
& \psi_{+}(x)=U_{2 p_{+}+1}(x)-U_{2 p_{+}-1}(x)-2, \\
& \psi_{-}(y)=U_{2 p_{-}+1}(y)-U_{2 p_{-}-1}(y)-2,
\end{aligned}
$$

and the equation

$$
U_{p_{+}+1}\left(\boldsymbol{C}_{+}\right)-U_{p_{+}-1}\left(\boldsymbol{C}_{+}\right)=U_{p_{-}+1}\left(\boldsymbol{C}_{-}\right)-U_{p_{--1}}\left(\boldsymbol{C}_{-}\right)
$$

(where both sides are actually equal to $(-1)^{p_{+}+p_{-}} 2 K^{p_{+} p_{-}}$). The statement in 4.2.7 is now immediate.

4.3. The $\mathfrak{g}_{p_{+}, p_{-}}$ribbon element. We recall that a ribbon Hopf algebra [30] is a quasitriangular Hopf algebra equipped with an invertible central element $\boldsymbol{v}$, called the ribbon element, such that

$$
S(\boldsymbol{v})=\boldsymbol{v}, \quad \epsilon(\boldsymbol{v})=1, \quad \Delta(\boldsymbol{v})=M^{-1}(\boldsymbol{v} \otimes \boldsymbol{v}) .
$$

In a ribbon Hopf algebra, $\boldsymbol{v}^{2}=\boldsymbol{u} S(\boldsymbol{u})$ and, in fact,

$$
\boldsymbol{v}=\boldsymbol{u} \boldsymbol{g}^{-1},
$$

where $\boldsymbol{g}$ is the balancing element and

$$
\boldsymbol{u}=\cdot\left((S \otimes \mathrm{id}) R_{21}\right)
$$

(where $\cdot(a \otimes b)=a b)$ is the canonical element [21]. 
4.3.1. Proposition. The $\mathfrak{g}_{p_{+}, p_{-}}$-ribbon element $\boldsymbol{v}$ is given by

$$
\boldsymbol{v}=\overline{\boldsymbol{v}} \boldsymbol{v}^{*}
$$

where

$$
\overline{\boldsymbol{v}}=\sum_{\left(r, r^{\prime}\right) \in \mathcal{I}} e^{2 i \pi \Delta_{r, r^{\prime}}} \boldsymbol{e}\left(r, r^{\prime}\right)
$$

with

$$
\Delta_{r, r^{\prime}}=\frac{\left(p_{+} r^{\prime}-p_{-} r\right)^{2}-\left(p_{+}-p_{-}\right)^{2}}{4 p_{+} p_{-}}
$$

(and $\boldsymbol{e}\left(r, r^{\prime}\right)$ being the primitive idempotents in the center), and

$$
\boldsymbol{v}^{*}=\left(\mathbf{1}+\frac{1}{p_{+}} \chi^{\nwarrow \searrow}(1,1)\right)\left(\mathbf{1}+\frac{1}{p_{-}} \chi^{\not{\swarrow}}(1,1)\right) .
$$

Proof. First, using the $R$-matrix in (4.2), we explicitly calculate the canonical element $\boldsymbol{u}$ in $\bar{D}(\mathcal{H})$ as

$$
\begin{aligned}
\boldsymbol{u}=\frac{1+i}{2 \sqrt{p_{+} p_{-}}} \sum_{m=0}^{p_{+}-1} \sum_{r=0}^{p_{+}-1} \sum_{n=0}^{p_{-}-1} \sum_{s=0}^{p_{-}-1} \frac{\left(\mathfrak{q}_{+}^{p_{-}}-\mathfrak{q}_{+}^{-p_{-}}\right)^{m}\left(\mathfrak{q}_{-}^{p_{+}}-\mathfrak{q}_{-}^{-p_{+}}\right)^{n}}{[m]_{+} ![n]_{-} !} \mathfrak{q}_{+}^{p_{-}\left(\frac{m(m+3)}{2}-\frac{r^{2}}{2}\right)} \mathfrak{q}_{-}^{p_{+}\left(\frac{n(n+3)}{2}-\frac{s^{2}}{2}\right)} \\
\times(-1)^{r s}\left(f_{+}^{m} K_{+}^{r-m} e_{+}^{m}\right)\left(\mathbf{1}+(-1)^{p_{+} s+p_{-}}(-i)^{p_{+} p_{-}} K^{p_{+} p_{-}}\right)\left(e_{-}^{n} K_{-}^{s+n} f_{-}^{n}\right) .
\end{aligned}
$$

The calculation involved the simple Gaussian sum

$$
\sum_{j=0}^{2 p_{+} p_{-}-1} e^{\frac{i \pi j^{2}}{2 p_{+} p_{-}}}=(1+i) \sqrt{p_{+} p_{-}} .
$$

The expression obtained shows, in particular, that $\boldsymbol{u}$ is an element of $\mathfrak{g}_{p_{+}, p_{-}}$, not of $\bar{D}(\mathcal{H})$. We then find the ribbon element in accordance with (4.25) and (3.6), as

$$
\boldsymbol{v}=\boldsymbol{u} K^{p_{--} p_{+}} .
$$

We next decompose the ribbon element $\boldsymbol{v}$ with respect to the basis of the Radford central elements. First, with the explicit expression for $v$, we find its action on the irreducible representations $\mathrm{X}_{r, r^{\prime}}^{+}$. An elementary calculation shows that the action of the ribbon element on irreducible modules is given by

$$
\begin{aligned}
\left.\boldsymbol{v}\right|_{\mathrm{X}_{r, r^{\prime}}^{+}}=(-1)^{r r^{\prime}+1} \mathfrak{q}_{+}^{p_{-} \frac{r^{2}-1}{2} \mathfrak{q}_{-}^{p_{+}}} & =e^{2 i \pi \Delta_{r, r^{\prime}},} \\
\left.\boldsymbol{v}\right|_{\mathrm{X}_{p_{+}, p_{-}}^{-}} & =e^{2 i \pi \Delta_{0, p_{-}}},
\end{aligned}
$$

thus associating $\Delta_{r, r^{\prime}}$ with each pair $\left(r, r^{\prime}\right) \in \mathcal{I}$.

With some more work (as for other decompositions obtained above; also cf. [5]), we calculate the nondiagonal part of the ribbon element action on projective modules and 
thus find the coefficients in the decomposition of the ribbon element with respect to the canonical central elements, as explained in B.2.2. With the notation in 3.2.3, we have

$$
\begin{aligned}
& \boldsymbol{v}= \sum_{\left(r, r^{\prime}\right) \in \mathcal{I}} e^{2 i \pi \Delta_{r, r^{\prime}}} \boldsymbol{e}\left(r, r^{\prime}\right)+ \\
&+\sum_{\left(r, r^{\prime}\right) \in \mathcal{I}_{1}}(-1)^{r} e^{2 i \pi \Delta_{r, r^{\prime}}} \frac{\mathfrak{q}_{-}^{p_{+} r^{\prime}}-\mathfrak{q}_{-}^{-p_{+} r^{\prime}}}{4 p_{-}^{2}}\left(r^{\prime} \boldsymbol{v}^{\swarrow}\left(r, r^{\prime}\right)-\left(p_{-}-r^{\prime}\right) \boldsymbol{v}^{\nearrow}\left(r, r^{\prime}\right)\right) \\
&+\sum_{\left(r, r^{\prime}\right) \in \mathcal{I}_{1}}(-1)^{r^{\prime}} e^{2 i \pi \Delta_{r, r^{\prime}}} \frac{\mathfrak{q}_{+}^{p_{-} r}-\mathfrak{q}_{+}^{-p_{-} r}}{4 p_{+}^{2}}\left(r \boldsymbol{v}^{\searrow}\left(r, r^{\prime}\right)-\left(p_{+}-r\right) \boldsymbol{v}^{\nwarrow}\left(r, r^{\prime}\right)\right) \\
&+\sum_{\left(r, r^{\prime}\right) \in \mathcal{I}_{1}}(-1)^{r+r^{\prime}} e^{2 i \pi \Delta_{r, r^{\prime}}} \frac{\left(\mathfrak{q}_{+}^{p_{-} r}-\mathfrak{q}_{+}^{-p_{-} r}\right)\left(\mathfrak{q}_{-}^{p_{+} r^{\prime}}-\mathfrak{q}_{-}^{-p_{+} r^{\prime}}\right)}{\sqrt{2}\left(p_{+} p_{-}\right)^{3 / 2}} \widehat{\varphi}\left(r, r^{\prime}\right) \\
& \quad-\sum_{r^{\prime}=1}^{p_{-}-1} e^{2 i \pi \Delta_{p_{+}, r^{\prime}}} \frac{(-1)^{p_{+}+p_{-}+r^{\prime}}\left(\mathfrak{q}_{-}^{p_{+} r^{\prime}}-\mathfrak{q}_{-}^{-p_{+} r^{\prime}}\right)}{\sqrt{2}\left(p_{+} p_{-}\right)^{3 / 2}} \widehat{\boldsymbol{\rho}}^{\square}\left(p_{+}, r^{\prime}\right) \\
& \quad-\sum_{r=1}^{p_{+}-1} e^{2 i \pi \Delta_{r, p_{-}}} \frac{(-1)^{p_{-}+p_{+}+r}\left(\mathfrak{q}_{+}^{p_{-} r}-\mathfrak{q}_{+}^{-p_{-} r}\right)}{\sqrt{2}\left(p_{+} p_{-}\right)^{3 / 2}} \widehat{\boldsymbol{\rho}}^{\square}\left(r, p_{-}\right) .
\end{aligned}
$$

The formulas in 3.2.1 of course allow expressing this through the Radford-map images, similarly to other decompositions of central elments, but the above form involving primitive idempotents shows that $\boldsymbol{v}$ factors into semisimple and unipotent (one-plus-nilpotent) parts as $\boldsymbol{v}=\overline{\boldsymbol{v}} \boldsymbol{v}^{*}$, with $\overline{\boldsymbol{v}}$ just as in the proposition and $\boldsymbol{v}^{*}$ given by $\mathbf{1}$ plus all the terms in (4.30) except the first sum, with all the factors $e^{2 i \pi \Delta_{r, r^{\prime}}}$ replaced by unity. The formulas in 4.2 then make it easy to verify that

$$
\boldsymbol{v}^{*}=\mathbf{1}+\frac{1}{p_{+} p_{-}} \chi^{\Downarrow}(1,1)+\frac{1}{p_{-}} \chi^{\nwarrow}(1,1)+\frac{1}{p_{+}} \chi^{\nwarrow \searrow}(1,1),
$$

whence the formula in the proposition follows immediately.

\section{5. $S L(2, \mathbb{Z})$-REPRESENTATIONS ON THE $\mathfrak{g}_{p_{+}, p_{-}}$CENTER}

The aim of this section is to prove Theorem 1.1. We define the $S L(2, \mathbb{Z})$-action on the $\mathfrak{g}_{p_{+}, p_{-}}$center $\mathrm{Z}$ as

$$
\begin{aligned}
& \mathcal{S}: a \mapsto \widehat{\phi}\left(\chi^{-1}(a)\right), \\
& \mathcal{T}: a \mapsto e^{-i \pi \frac{c}{12}} \mathcal{S}\left(\boldsymbol{v} \mathcal{S}^{-1}(a)\right),
\end{aligned}
$$

which follows [17, 18, 19] with insignificant variations. Here,

$$
c=13-6 \frac{p_{+}}{p_{-}}-6 \frac{p_{-}}{p_{+}},
$$

which is the central charge of the $\left(p_{+}, p_{-}\right)$conformal field theory model. 
5.1. $S L(2, \mathbb{Z})$-adapted bases in the center. We have two "distinguished" bases in the center Z of $\mathfrak{g}_{p_{+}, p_{-}}$, associated with the Radford and the Drinfeld maps. The "Radford" basis is given in (3.15). The "Drinfeld" basis consists of the $2 p_{+} p_{-}+\left(p_{+}-1\right) p_{-}+$ $p_{+}\left(p_{-}-1\right)+\frac{1}{2}\left(p_{+}-1\right)\left(p_{-}-1\right)$ elements

$$
\begin{array}{lll}
\chi^{ \pm}\left(r, r^{\prime}\right), & 1 \leqslant r \leqslant p_{+}, \quad 1 \leqslant r^{\prime} \leqslant p_{-}, & \\
\chi^{\nwarrow \searrow}\left(r, r^{\prime}\right), & 1 \leqslant r \leqslant p_{+}-1, \quad \chi^{\varkappa 火}\left(r, r^{\prime}\right), & 1 \leqslant r \leqslant p_{+}, \\
& 1 \leqslant r^{\prime} \leqslant p_{-}, & 1 \leqslant r^{\prime} \leqslant p_{-}-1, \\
\chi^{\downarrow}\left(r, r^{\prime}\right), & \left(r, r^{\prime}\right) \in \mathcal{I}_{1} . &
\end{array}
$$

From the definitions, the $\mathcal{S}$ mapping relates the two bases as

$$
\begin{aligned}
\mathcal{S}^{-1}\left(\widehat{\phi}^{ \pm}\left(r, r^{\prime}\right)\right) & =\chi^{ \pm}\left(r, r^{\prime}\right), \\
\mathcal{S}^{-1}\left(\widehat{\phi}^{\nwarrow}\left(r, r^{\prime}\right)\right) & =\chi^{\nwarrow \searrow}\left(r, r^{\prime}\right), \\
\mathcal{S}^{-1}\left(\widehat{\phi}^{\nwarrow \searrow}\left(r, r^{\prime}\right)\right) & =\chi^{\nwarrow}\left(r, r^{\prime}\right), \\
\mathcal{S}^{-1}\left(\widehat{\phi}^{\Uparrow}\left(r, r^{\prime}\right)\right) & =\chi^{\Downarrow}\left(r, r^{\prime}\right) .
\end{aligned}
$$

In accordance with [17],

$$
\left.\mathcal{S}^{2}\right|_{z}=\mathrm{id}
$$

which, clearly, requires a proper choice of normalization in our formulas, most easily checked by calculating $\mathcal{S}^{-1}(\mathbf{1})=\mathcal{S}^{-1}\left(\chi^{+}(1,1)\right)=\widehat{\boldsymbol{\phi}}^{+}(1,1)=\boldsymbol{\Lambda}$. (As we have noted, this fixes the value of $\zeta$ in (3.5).)

5.2. Modular group action on the center. We now prove the most tedious part of Theorem 1.1, the structure of the $S L(2, \mathbb{Z})$ representation on Z in Claim 3 , The proof amounts to a series of lemmas.

5.2.1. Lemma. For $\left(r, r^{\prime}\right) \in \mathcal{I}_{1}$, the elements

$$
\widehat{\varphi}^{\times}\left(r, r^{\prime}\right)=\widehat{\varphi}^{\nwarrow /}\left(r, r^{\prime}\right)-\widehat{\varphi}^{\nwarrow \searrow}\left(r, r^{\prime}\right)
$$

(see the notation in (4.18)) span a $\frac{1}{2}\left(p_{+}-1\right)\left(p_{-}-1\right)$-dimensional $S L(2, \mathbb{Z})$-subrepresentation in Z. Moreover, this representation is isomorphic to $R_{\min }$ in (1.3). 8

Proof. First, the formulas

$$
\begin{aligned}
& \boldsymbol{v} \widehat{\varphi}^{\not{X}}\left(r, r^{\prime}\right)=e^{2 i \pi \Delta_{r, r^{\prime}}} \widehat{\boldsymbol{\varphi}}^{\not{X}}\left(r, r^{\prime}\right)+e^{2 i \pi \Delta_{r, r^{\prime}}} \widehat{\boldsymbol{\varphi}}\left(r, r^{\prime}\right),
\end{aligned}
$$

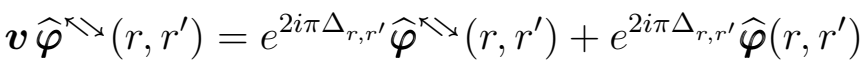

(which are a direct consequence of 3.2.1 and (4.30) ) show that

$$
\boldsymbol{v} \widehat{\boldsymbol{\varphi}}^{\times}\left(r, r^{\prime}\right)=e^{2 i \pi \Delta_{r, r^{\prime}}} \widehat{\boldsymbol{\varphi}}^{\times}\left(r, r^{\prime}\right) .
$$

\footnotetext{
${ }^{8}$ We remind the reader that this is the $S L(2, \mathbb{Z})$ representation in the minimal $\left(p_{+}, p_{-}\right)$conformal field theory model.
} 
Second, we find $\mathcal{S}\left(\widehat{\varphi}^{\not{x}}\left(r, r^{\prime}\right)\right)$ from Eqs. (5.3) and (4.13), which imply

$$
\begin{aligned}
& \mathcal{S}\left(\widehat{\varphi}^{\nwarrow}\left(r, r^{\prime}\right)\right)=(-1)^{r^{\prime}}\left(\left(p_{-}-r^{\prime}\right) \chi^{\nwarrow \searrow}\left(r, r^{\prime}\right)+(-1)^{p_{-}} r^{\prime} \chi^{\nwarrow \searrow}\left(p_{+}-r, p_{-}-r^{\prime}\right)\right) \\
& \quad=-\frac{1}{\sqrt{2 p_{+} p_{-}}} \sum_{\left(s, s^{\prime}\right) \in \mathcal{I}_{1}}(-1)^{r s^{\prime}+r^{\prime} s}\left(\mathfrak{q}_{+}^{p_{-} r s}-\mathfrak{q}_{+}^{-p_{-} r s}\right)\left(\mathfrak{q}_{-}^{p_{+} r^{\prime} s^{\prime}}-\mathfrak{q}_{-}^{-p_{+} r^{\prime} s^{\prime}}\right) \widehat{\varphi}^{\nwarrow \searrow}\left(s, s^{\prime}\right),
\end{aligned}
$$

where we also used (3.12). With a similar expression for $\mathcal{S}\left(\widehat{\varphi}^{\nwarrow \searrow}\left(r, r^{\prime}\right)\right)$, we have

$$
\mathcal{S}\left(\widehat{\varphi}^{\times}\left(r, r^{\prime}\right)\right)=\frac{1}{\sqrt{2 p_{+} p_{-}}} \sum_{\left(s, s^{\prime}\right) \in \mathcal{I}_{1}}(-1)^{r s^{\prime}+r^{\prime} s}\left(\mathfrak{q}_{+}^{p_{-} r s}-\mathfrak{q}_{+}^{-p_{-} r s}\right)\left(\mathfrak{q}_{-}^{p_{-} r^{\prime} s^{\prime}}-\mathfrak{q}_{-}^{-p_{-} r^{\prime} s^{\prime}}\right) \widehat{\varphi}^{\times}\left(s, s^{\prime}\right) .
$$

The lemma is proved.

We once again recall the notation in (4.18) and set

$$
\widehat{\boldsymbol{\psi}}\left(r, r^{\prime}\right)=\widehat{\varphi}^{\not{\Sigma}}\left(r, r^{\prime}\right)+\widehat{\varphi}^{\nwarrow \searrow}\left(r, r^{\prime}\right) .
$$

5.2.2. Lemma. The elements $\widehat{\boldsymbol{\phi}}^{\uparrow}\left(r, r^{\prime}\right), \widehat{\boldsymbol{\psi}}\left(r, r^{\prime}\right)$, and $\widehat{\varphi}\left(r, r^{\prime}\right)$ with $\left(r, r^{\prime}\right) \in \mathcal{I}_{1}$ span a $\frac{3}{2}\left(p_{+}-1\right)\left(p_{-}-1\right)$-dimensional $S L(2, \mathbb{Z})$-subrepresentation $\mathcal{R}$ in $\mathrm{Z}$ and, moreover,

$$
\mathcal{R}=\mathbb{C}^{3} \otimes R_{\text {min }},
$$

where $\mathbb{C}^{3}$ is the symmetrized square of the standard two-dimensional representation and $R_{\min }$ is the representation in (1.3).

Proof. Clearly,

$$
\boldsymbol{v} \widehat{\varphi}\left(r, r^{\prime}\right)=e^{2 i \pi \Delta_{r, r^{\prime}}} \widehat{\varphi}\left(r, r^{\prime}\right)
$$

from (4.30) and 3.2.1. We next evaluate the action of the ribbon element on $\widehat{\phi}^{\uparrow}\left(r, r^{\prime}\right)$ using 3.2.1, which gives

$$
\boldsymbol{v} \widehat{\boldsymbol{\phi}}^{\Uparrow}\left(r, r^{\prime}\right)=e^{2 i \pi \Delta_{r, r^{\prime}}}\left(\widehat{\boldsymbol{\phi}}^{\uparrow}\left(r, r^{\prime}\right)+(-1)^{r+r^{\prime}} \widehat{\boldsymbol{\varphi}}\left(r, r^{\prime}\right)+(-1)^{r+r^{\prime}} \widehat{\boldsymbol{\psi}}\left(r, r^{\prime}\right)\right) \text {. }
$$

Next, a similar calculation yields

$$
\boldsymbol{v} \widehat{\boldsymbol{\psi}}\left(r, r^{\prime}\right)=e^{2 i \pi \Delta_{r, r^{\prime}}}\left(\widehat{\boldsymbol{\psi}}\left(r, r^{\prime}\right)+2 \widehat{\boldsymbol{\varphi}}\left(r, r^{\prime}\right)\right)
$$

As regards the $\mathcal{S}$ action, we recall (5.5) and (4.17), which we rewrite as

$$
\mathcal{S}^{-1}\left(\widehat{\boldsymbol{\phi}}^{\uparrow}\left(r, r^{\prime}\right)\right)=\frac{(-1)^{r+r^{\prime}}}{\sqrt{2 p_{+} p_{-}}} \sum_{\left(s, s^{\prime}\right) \in \mathcal{I}_{1}}(-1)^{r s^{\prime}+r^{\prime} s}\left(\mathfrak{q}_{+}^{p_{-} r s}-\mathfrak{q}_{+}^{-p_{-} r s}\right)\left(\mathfrak{q}_{-}^{p_{+} r^{\prime} s^{\prime}}-\mathfrak{q}_{-}^{-p_{+} r^{\prime} s^{\prime}}\right) \widehat{\varphi}\left(s, s^{\prime}\right) \text {. }
$$

Next, repeating the calculation in the proof of 5.2.1, we obtain

$$
\mathcal{S}^{-1}\left(\widehat{\boldsymbol{\psi}}\left(r, r^{\prime}\right)\right)=-\frac{1}{\sqrt{2 p_{+} p_{-}}} \sum_{\left(s, s^{\prime}\right) \in \mathcal{I}_{1}}(-1)^{r s^{\prime}+r^{\prime} s}\left(\mathfrak{q}_{+}^{p_{-} r s}-\mathfrak{q}_{+}^{-p_{-} r s}\right)\left(\mathfrak{q}_{-}^{p_{+} r^{\prime} s^{\prime}}-\mathfrak{q}_{-}^{-p_{+} r^{\prime} s^{\prime}}\right) \widehat{\boldsymbol{\psi}}\left(s, s^{\prime}\right) .
$$

This completes the proof.

5.2.3. Lemma. For $\left(r, r^{\prime}\right) \in \mathcal{I}_{\square}$, the elements $\widehat{\boldsymbol{\rho}}^{\square}\left(r, r^{\prime}\right)$ (see (13.22) and (3.23) $)$ and $\widehat{\varphi}^{\square}\left(r, r^{\prime}\right)$ (see (3.26) and (3.27) ) span $a\left(p_{+}-1\right)\left(p_{-}+1\right)$-dimensional $S L(2, \mathbb{Z})$-subrep- 
resentation $\mathcal{R}_{\square}$ in $\mathrm{Z}$. Moreover, its structure is

$$
\mathcal{R}_{\square}=\mathbb{C}^{2} \otimes R_{\square},
$$

where $\mathbb{C}^{2}$ is the standard two-dimensional $S L(2, \mathbb{Z})$ representation and $R_{\square}$ is the representation in (1.3).

Proof. We first calculate the ribbon action. From (4.30) and 3.2.1, we have

$$
\boldsymbol{v} \widehat{\boldsymbol{\varphi}}^{\square}\left(r, r^{\prime}\right)=e^{2 i \pi \Delta_{r, r^{\prime}}} \widehat{\boldsymbol{\varphi}}^{\square}\left(r, r^{\prime}\right)+e^{2 i \pi \Delta_{r, r^{\prime}}} \widehat{\boldsymbol{\rho}}^{\square}\left(r, r^{\prime}\right)
$$

for $\left(r, r^{\prime}\right) \in \mathcal{I}_{1}$, and

$$
\boldsymbol{v} \widehat{\boldsymbol{\varphi}}^{\square}\left(r, p_{-}\right)=e^{2 i \pi \Delta_{r, p_{-}}} \widehat{\boldsymbol{\varphi}}^{\square}\left(r, p_{-}\right)+e^{2 i \pi \Delta_{r, p_{-}}} \widehat{\boldsymbol{\rho}}^{\square}\left(r, p_{-}\right) .
$$

From (3.22) and (3.23), evidently,

$$
\boldsymbol{v} \widehat{\boldsymbol{\rho}}^{\square}\left(r, r^{\prime}\right)=e^{2 i \pi \Delta_{r, r^{\prime}}} \widehat{\boldsymbol{\rho}}^{\square}\left(r, r^{\prime}\right)
$$

for all the $\widehat{\rho}^{\square}$ elements in the lemma.

Next, the $\mathcal{S}$-action is calculated as

$$
\begin{aligned}
\mathcal{S}^{-1}\left(\widehat{\boldsymbol{\varphi}}^{\square}\left(r, r^{\prime}\right)\right) & =(-1)^{r^{\prime}}\left(\chi^{\nwarrow \searrow}\left(r, r^{\prime}\right)-(-1)^{p_{-}} \chi^{\nwarrow \searrow}\left(p_{+}-r, p_{-}-r^{\prime}\right)\right) \\
& =\frac{1}{\sqrt{2 p_{+} p_{-}}} \sum_{s=1}^{p_{+}-1}(-1)^{r^{\prime}\left(s+p_{+}\right)+p_{-} r}\left(\mathfrak{q}_{+}^{p_{-} r s}-\mathfrak{q}_{+}^{-p_{-} r s}\right) \widehat{\boldsymbol{\rho}}^{\square}\left(s, p_{-}\right) \\
& +\frac{1}{\sqrt{2 p_{+} p_{-}}} \sum_{\left(s, s^{\prime}\right) \in \mathcal{I}_{1}}(-1)^{r s^{\prime}+r^{\prime} s}\left(\mathfrak{q}_{+}^{p_{-} r s}-\mathfrak{q}_{+}^{-p_{-} r s}\right)\left(\mathfrak{q}_{-}^{p_{+} r^{\prime} s^{\prime}}+\mathfrak{q}_{-}^{-p_{+} r^{\prime} s^{\prime}}\right) \widehat{\boldsymbol{\rho}}^{\square}\left(s, s^{\prime}\right),
\end{aligned}
$$

where $\left(r, r^{\prime}\right) \in \mathcal{I}_{1}$ and we used (4.13), and, similarly,

$$
\begin{aligned}
\mathcal{S}^{-1}\left(\widehat{\boldsymbol{\varphi}}^{\square}\left(r, p_{-}\right)\right) & =(-1)^{p_{-}} \chi^{\nwarrow \searrow}\left(r, p_{-}\right) \\
= & \frac{1}{\sqrt{2 p_{+} p_{-}}} \sum_{s=1}^{p_{+}-1}(-1)^{p_{-}\left(s+p_{+}+r\right)}\left(\mathfrak{q}_{+}^{p_{-} r s}-\mathfrak{q}_{+}^{-p_{-} r s}\right) \widehat{\boldsymbol{\rho}}^{\square}\left(s, p_{-}\right) \\
& \quad+\frac{1}{\sqrt{2 p_{+} p_{-}}} \sum_{\left(s, s^{\prime}\right) \in \mathcal{I}_{1}} 2(-1)^{\left(p_{+}+r\right) s^{\prime}+p_{-} s}\left(\mathfrak{q}_{+}^{p_{-} r s}-\mathfrak{q}_{+}^{-p_{-} r s}\right) \widehat{\boldsymbol{\rho}}^{\square}\left(s, s^{\prime}\right) .
\end{aligned}
$$

Conversely, from (4.8) we have

$$
\begin{aligned}
& \mathcal{S}^{-1}\left(\widehat{\boldsymbol{\rho}}^{\square}\left(r, r^{\prime}\right)\right)= \\
&=\left(p_{+}-r\right)\left(\chi^{+}\left(r, r^{\prime}\right)+\chi^{-}\left(r, p_{-}-r^{\prime}\right)\right)-r\left(\chi^{-}\left(p_{+}-r, r^{\prime}\right)+\chi^{+}\left(p_{+}-r, p_{-}-r^{\prime}\right)\right) \\
&=-\frac{1}{\sqrt{2 p_{+} p_{-}}}\left(\sum_{s=1}^{p_{+}-1}(-1)^{r p_{-}+r^{\prime}\left(s+p_{+}\right)}\left(\mathfrak{q}_{+}^{p_{-} r s}-\mathfrak{q}_{+}^{-p_{-} r s}\right) \widehat{\boldsymbol{\varphi}}^{\square}\left(s, p_{-}\right)\right. \\
&\left.\quad+\sum_{\left(s, s^{\prime}\right) \in \mathcal{I}_{1}}(-1)^{r s^{\prime}+r^{\prime} s}\left(\mathfrak{q}_{+}^{p_{-} r s}-\mathfrak{q}_{+}^{-p_{-} r s}\right)\left(\mathfrak{q}_{-}^{p_{+} r^{\prime} s^{\prime}}+\mathfrak{q}_{-}^{-p_{+} r^{\prime} s^{\prime}}\right) \widehat{\boldsymbol{\varphi}}^{\square}\left(s, s^{\prime}\right)\right),
\end{aligned}
$$


which completes the proof.

Totally similarly, we establish the following lemma.

5.2.4. Lemma. For $\left(r, r^{\prime}\right) \in \mathcal{I}_{\square}$, the elements $\widehat{\boldsymbol{\rho}}^{\square}\left(r, r^{\prime}\right)$ (see (3.24) and (3.25)) and $\widehat{\varphi}^{\square}\left(r, r^{\prime}\right)$ (see (3.28) and (3.29) ) span a $\left(p_{+}+1\right)\left(p_{-}-1\right)$-dimensional $S L(2, \mathbb{Z})$-subrepresentation $\mathcal{R}_{\square}$ in Z. Moreover, its structure is

$$
\mathcal{R}_{\square}=\mathbb{C}^{2} \otimes R_{\square},
$$

where $\mathbb{C}^{2}$ is the standard two-dimensional $S L(2, \mathbb{Z})$ representation and $R_{\square}$ is the representation in (1.3).

To formulate our next (and final) lemma, we recall the notation in (3.16)-(3.20).

5.2.5. Lemma. The elements $\widehat{\varkappa}\left(r, r^{\prime}\right),\left(r, r^{\prime}\right) \in \mathcal{I}$, span a $\frac{1}{2}\left(p_{+}+1\right)\left(p_{-}+1\right)$-dimensional $S L(2, \mathbb{Z})$-subrepresentation $R_{\text {proj }}$ in $\mathbf{Z}$.

Proof. Clearly, for $\left(r, r^{\prime}\right) \in \mathcal{I}$, the ribbon element acts as

$$
\boldsymbol{v} \widehat{\varkappa}\left(r, r^{\prime}\right)=e^{2 i \pi \Delta_{r, r^{\prime}}} \widehat{\boldsymbol{\varkappa}}\left(r, r^{\prime}\right) \text {. }
$$

As regards the $\mathcal{S}$ action, we recall (5.2) and (4.8), which we rewrite, for $\left(r, r^{\prime}\right) \in \mathcal{I}_{1}$ and using notation (3.16) $-(3.20)$, as

$$
\begin{aligned}
\mathcal{S}^{-1}\left(\widehat{\varkappa}\left(r, r^{\prime}\right)\right)= & \chi^{+}\left(r, r^{\prime}\right)+\chi^{-}\left(p_{+}-r, r^{\prime}\right)+\chi^{-}\left(r, p_{-}-r^{\prime}\right)+\chi^{+}\left(p_{+}-r, p_{-}-r^{\prime}\right) \\
= & \frac{1}{\sqrt{2 p_{+} p_{-}}}\left(\widehat{\varkappa}\left(p_{+}, p_{-}\right)+(-1)^{r p_{-}+r^{\prime} p_{+}} \widehat{\varkappa}\left(0, p_{-}\right)\right. \\
& +\sum_{s=1}^{p_{+}-1}(-1)^{r p_{-}+r^{\prime}\left(p_{+}-s\right)}\left(\mathfrak{q}_{+}^{p_{-} r s}+\mathfrak{q}_{+}^{-p_{-} r s}\right) \widehat{\varkappa}\left(s, p_{-}\right) \\
& +\sum_{s^{\prime}=1}^{p_{-}-1}(-1)^{r^{\prime} p_{+}+r\left(p_{-}-s^{\prime}\right)}\left(\mathfrak{q}_{-}^{p_{+} r^{\prime} s^{\prime}}+\mathfrak{q}_{+}^{-p_{+} r^{\prime} s^{\prime}}\right) \widehat{\varkappa}\left(p_{+}, s^{\prime}\right) \\
& \left.+\sum_{\left(s, s^{\prime}\right) \in \mathcal{I}_{1}}(-1)^{r s^{\prime}+r^{\prime} s}\left(\mathfrak{q}_{+}^{p_{-} r s}+\mathfrak{q}_{+}^{-p_{-} r s}\right)\left(\mathfrak{q}_{-}^{p_{+} r^{\prime} s^{\prime}}+\mathfrak{q}_{+}^{-p_{+} r^{\prime} s^{\prime}}\right) \widehat{\varkappa}\left(s, s^{\prime}\right)\right),
\end{aligned}
$$

and similarly for $\mathcal{S}^{-1}\left(\widehat{\varkappa}\left(r, p_{-}\right)\right), \mathcal{S}^{-1}\left(\widehat{\varkappa}\left(p_{+}, r^{\prime}\right)\right)$, and $\mathcal{S}^{-1}\left(\widehat{\phi}^{ \pm}\left(p_{+}, p_{-}\right)\right)$. The lemma is proved.

This completes the proof of Claim 3 in 1.1

5.2.6. Claim 4 is now obvious if we recall from (4.8) that the Drinfeld-map image of the Grothendieck ring in $\mathrm{Z}$ is spanned by

$$
\begin{array}{llll}
\widehat{\phi}^{\Uparrow}\left(r, r^{\prime}\right), & \left(r, r^{\prime}\right) \in \mathcal{I}_{1}, & \widehat{\varkappa}\left(r, r^{\prime}\right), & \left(r, r^{\prime}\right) \in \mathcal{I}, \\
\widehat{\varphi}^{\square}\left(r, r^{\prime}\right), & \left(r, r^{\prime}\right) \in \mathcal{I}_{\square}, & \widehat{\varphi}^{\square}\left(r, r^{\prime}\right), & \left(r, r^{\prime}\right) \in \mathcal{I}_{\square} .
\end{array}
$$


5.3. Comparison with the $S L(2, \mathbb{Z})$-representation on generalized characters of the $W$-algebra. Claim 5 in 1.1 is established by direct comparison with formulas in [22, Lemmas 4.3 and 4.4]. First, with 5.2.1, we define

$$
\chi_{r, r^{\prime}}=\chi^{\nwarrow \searrow}\left(r, r^{\prime}\right)-\chi^{\nwarrow \swarrow}\left(r, r^{\prime}\right)=\mathcal{S} \widehat{\varphi}^{\times}\left(r, r^{\prime}\right), \quad\left(r, r^{\prime}\right) \in \mathcal{I}_{1} .
$$

Then the $\chi_{r, r^{\prime}}$ transform under $\mathcal{S}$ and $\mathcal{T}$ in (5.1) as the functions $\chi_{r, r^{\prime}}(\tau)$ in [22] transform under $\tau \mapsto-\frac{1}{\tau}$ and $\tau \mapsto \tau+1$. (In particular, $\mathcal{T} \chi_{r, r^{\prime}}=e^{-i \pi \frac{c}{12}} \mathcal{S}\left(\boldsymbol{v} \widehat{\varphi}^{\times}\left(r, r^{\prime}\right)\right)=$ $e^{-i \pi \frac{c}{12}} e^{2 i \pi \Delta_{r, r^{\prime}}} \chi_{r, r^{\prime}}$.)

Next, with 5.2.2, we define

$$
\begin{aligned}
& \rho_{r, r^{\prime}}=\mathcal{S} \widehat{\boldsymbol{\varphi}}\left(r, r^{\prime}\right)=\frac{-2 \sqrt{2}}{\sqrt{p_{+} p_{-}}} \sum_{\left(s, s^{\prime}\right) \in \mathcal{I}_{1}}(-1)^{(r+1) s^{\prime}+\left(r^{\prime}+1\right) s} \sin \frac{\pi p_{-} r s}{p_{+}} \sin \frac{\pi p_{+} r^{\prime} s^{\prime}}{p_{-}} \widehat{\boldsymbol{\phi}}^{\Uparrow}\left(s, s^{\prime}\right), \\
& \psi_{r, r^{\prime}}=\mathcal{S} \widehat{\boldsymbol{\psi}}\left(r, r^{\prime}\right)=\frac{2 \sqrt{2}}{\sqrt{p_{+} p_{-}}} \sum_{\left(s, s^{\prime}\right) \in \mathcal{I}_{1}}(-1)^{r s^{\prime}+r^{\prime} s} \sin \frac{\pi p_{-} r s}{p_{+}} \sin \frac{\pi p_{+} r^{\prime} s^{\prime}}{p_{-}} \widehat{\boldsymbol{\psi}}\left(s, s^{\prime}\right), \\
& \varphi_{r, r^{\prime}}=(-1)^{r+r^{\prime}} \boldsymbol{\chi}^{\Downarrow}\left(r, r^{\prime}\right),
\end{aligned}
$$

where $\left(r, r^{\prime}\right) \in \mathcal{I}_{1}$. Then $\rho_{r, r^{\prime}}, \psi_{r, r^{\prime}}$, and $\varphi_{r, r^{\prime}}$ transform under $\mathcal{S}$ and $\mathcal{T}$ in (5.1) as the respective functions $\rho_{r, r^{\prime}}(\tau), \psi_{r, r^{\prime}}(\tau)$, and $\varphi_{r, r^{\prime}}(\tau)$ in [22] transform under $\tau \mapsto-\frac{1}{\tau}$ and $\tau \mapsto \tau+1$. (In particular, $\mathcal{T} \psi_{r, r^{\prime}}=e^{-i \pi \frac{c}{12}} e^{2 i \pi \Delta_{r, r^{\prime}}}\left(\psi_{r, r^{\prime}}+2 \rho_{r, r^{\prime}}\right)$ and $\mathcal{T} \varphi_{r, r^{\prime}}=$ $e^{-i \pi \frac{c}{12}} e^{2 i \pi \Delta_{r, r^{\prime}}}\left(\varphi_{r, r^{\prime}}+\psi_{r, r^{\prime}}+\rho_{r, r^{\prime}}\right)$.)

Similarly, with 5.2.3, we set

$$
\begin{array}{rlrl}
\varphi_{r, r^{\prime}}^{\square} & =-(-1)^{r^{\prime}} \chi^{\nwarrow \searrow}\left(r, r^{\prime}\right)+(-1)^{p_{-}+r^{\prime}} \chi^{\nwarrow \searrow}\left(p_{+}-r, p_{-}-r^{\prime}\right), & & \left(r, r^{\prime}\right) \in \mathcal{I}_{1}, \\
\varphi_{r, 0}^{\square}=(-1)^{p_{-}} \chi^{\nwarrow \searrow}\left(p_{+}-r, p_{-}\right), & & 1 \leqslant r \leqslant p_{+}-1,
\end{array}
$$

and

$$
\begin{aligned}
& \rho_{r, r^{\prime}}^{\square}=-\mathcal{S} \widehat{\boldsymbol{\rho}}^{\square}\left(r, r^{\prime}\right)=r\left(\chi^{-}\left(p_{+}-r, r^{\prime}\right)+\chi^{+}\left(p_{+}-r, p_{-}-r^{\prime}\right)\right) \\
& -\left(p_{+}-r\right)\left(\chi^{+}\left(r, r^{\prime}\right)+\chi^{-}\left(r, p_{-}-r^{\prime}\right)\right), \quad\left(r, r^{\prime}\right) \in \mathcal{I}_{1}, \\
& \rho_{r, 0}^{\square}=\mathcal{S} \widehat{\boldsymbol{\rho}}^{\square}\left(p_{+}-r, p_{-}\right)=r \boldsymbol{\chi}^{+}\left(p_{+}-r, p_{-}\right)-\left(p_{+}-r\right) \chi^{-}\left(r, p_{-}\right) . \quad 1 \leqslant r \leqslant p_{+}-1 .
\end{aligned}
$$

Then the $\rho_{r, r^{\prime}}^{\square}$ and $\varphi_{r, r^{\prime}}^{\square}$ thus defined transform under $\mathcal{S}$ and $\mathcal{T}$ in (5.1) as the respective functions $\rho_{r, r^{\prime}}^{\square}(\tau)$ and $\varphi_{r, r^{\prime}}^{\square}(\tau)$ in [22] transform under $\tau \mapsto-\frac{1}{\tau}$ and $\tau \mapsto \tau+1$.

We give some more details here, mainly because of a minor difference between the conventions in this paper and in [5]. First, from the formulas in the proof of [5.2.3, it follows that

$$
\begin{aligned}
\mathcal{S} \varphi_{r, r^{\prime}}^{\square}=\frac{\sqrt{2} i}{\sqrt{p_{+} p_{-}}}\left(\sum_{s=1}^{p_{+}-1}(-1)^{s r^{\prime}} \sin \frac{\pi p_{-} r s}{p_{+}} \rho_{s, 0}^{\square}\right. & \\
& \left.+\sum_{\left(s, s^{\prime}\right) \in \mathcal{J}_{1}} 2(-1)^{r s^{\prime}+s r^{\prime}} \sin \frac{\pi p_{-} r s}{p_{+}} \cos \frac{\pi p_{+} r^{\prime} s^{\prime}}{p_{-}} \rho_{s, s^{\prime}}^{\square}\right),
\end{aligned}
$$


for all the $\varphi^{\square}$ defined above, which coincides with the $\mathcal{S}$-transformation formula for $\varphi_{r, r^{\prime}}^{\square}(\tau)$ found in [22]. Next, from the last formula in the proof of 5.2.3 and formulas (3.26) - (3.27), it follows that

$$
\begin{aligned}
\mathcal{S} \rho_{r, r^{\prime}}^{\square}=\frac{-\sqrt{2} i}{\sqrt{p_{+} p_{-}}}\left(\sum_{s=1}^{p_{+}-1}(-1)^{s r^{\prime}} \sin \frac{\pi p_{-} r s}{p_{+}} \varphi_{s, 0}^{\square}\right. & \\
& \left.+\sum_{\left(s, s^{\prime}\right) \in \mathcal{J}_{1}} 2(-1)^{r s^{\prime}+s r^{\prime}} \sin \frac{\pi p_{-} r s}{p_{+}} \cos \frac{\pi p_{+} r^{\prime} s^{\prime}}{p_{-}} \varphi_{s, s^{\prime}}^{\square}\right)
\end{aligned}
$$

for $\left(r, r^{\prime}\right) \in \mathcal{I}_{1}$, which is also the formula for $\rho_{r, r^{\prime}}^{\square}\left(-\frac{1}{\tau}\right)$ in [22]. Finally, it follows from the definitions (see (3.23) $)$ that $\mathcal{S} \rho_{r, 0}^{\square}=\widehat{\boldsymbol{\rho}}^{\square}\left(p_{+}-r, p_{-}\right)=r \mathcal{S} \chi^{+}\left(p_{+}-r, p_{-}\right)-$ $\left(p_{+}-r\right) \mathcal{S} \chi^{-}\left(r, p_{-}\right)$. Using (4.8) now gives

$$
\begin{array}{r}
r \chi^{+}\left(p_{+}-r, p_{-}\right)-\left(p_{+}-r\right) \chi^{-}\left(r, p_{-}\right)=\frac{\sqrt{2} i}{\sqrt{p_{+} p_{-}}}\left(\sum_{s=1}^{p_{+}-1}(-1)^{(r-1) p_{-}} \sin \frac{\pi p_{-} r s}{p_{+}} \widehat{\boldsymbol{\phi}}^{\boldsymbol{\Sigma}}\left(s, p_{-}\right)\right. \\
\left.+\sum_{\left(s, s^{\prime}\right) \in \mathcal{I}_{1}} 2(-1)^{r s^{\prime}} \sin \frac{\pi p_{-} r s}{p_{+}} \widehat{\varphi}^{\square}\left(s, s^{\prime}\right)\right),
\end{array}
$$

which readily shows that $\mathcal{S} \rho_{r, 0}^{\square}$ is given by the above formula for $\mathcal{S} \rho_{r, r^{\prime}}^{\square}$ at $r^{\prime}=0$ (as in [22]).

It next follows from the ribbon element action in $\mathbf{5 . 2 . 3}$ that

$$
\mathcal{T} \varphi_{r, r^{\prime}}^{\square}=e^{-i \pi \frac{c}{12}} e^{2 i \pi \Delta_{r, r^{\prime}}}\left(\varphi_{r, r^{\prime}}^{\square}+\rho_{r, r^{\prime}}^{\square}\right)
$$

for all $\varphi_{r, r^{\prime}}^{\square}$ defined above (for $\varphi_{r, 0}^{\square}$, the simple derivation involves the identity $\Delta_{r, 0}=$ $\Delta_{p_{+}-r, p_{-}}$, see (4.29)). Equally easily, 5.2.3 also implies that

$$
\mathcal{T} \rho_{r, r^{\prime}}^{\square}=e^{-i \pi \frac{c}{12}} e^{2 i \pi \Delta_{r, r^{\prime}}} \rho_{r, r^{\prime}}^{\square}
$$

for all $\rho_{r, r^{\prime}}^{\square}$ defined above.

The correspondence involving the central elements in 5.2.4 is totally similar. Finally, for the central elements in 5.2.5, the correspondence is also established straightforwardly, and we omit the details.

5.4. Factorization of the $S L(2, \mathbb{Z})$ action. We next consider factorization Claim 2 in 1.1 . As in a simpler case studied in [5], this factorization is related to the factorization of the ribbon element in (4.27). We first note that with the $M$-matrix expressed as $M=$ $(\boldsymbol{v} \otimes \boldsymbol{v}) \Delta(\boldsymbol{v})^{-1}$, it readily follows [17] that

$$
\mathcal{S}(\boldsymbol{v})=\boldsymbol{v}^{-1}
$$

We now define a map $\boldsymbol{\xi}: \mathrm{Ch} \rightarrow \mathrm{Z}$, which is an isomorphism of vector spaces and intertwines the coadjoint and adjoint actions of $\mathfrak{g}_{p_{+}, p_{-}}$, as

$$
\boldsymbol{\xi}(\beta)=(\beta \otimes \mathrm{id}) M^{*},
$$


where

$$
M^{*}=\left(\boldsymbol{v}^{*} \otimes \boldsymbol{v}^{*}\right) \Delta\left(\mathcal{S}\left(\boldsymbol{v}^{*}\right)\right)
$$

From the $\mathcal{S}$-transformation formulas in $\mathbf{5 . 2}$, we immediately obtain

$$
\mathcal{S}\left(\boldsymbol{v}^{*}\right)=\boldsymbol{\Lambda}+\frac{1}{p_{+} p_{-}} \widehat{\boldsymbol{\phi}}^{\Uparrow \uparrow}(1,1)+\frac{1}{p_{+}} \widehat{\boldsymbol{\phi}}^{\nwarrow}(1,1)+\frac{1}{p_{-}} \widehat{\boldsymbol{\phi}}^{\nwarrow \searrow}(1,1) .
$$

The maps $Z \rightarrow Z$ given by

$$
\mathcal{S}^{*}=\widehat{\phi} \circ \boldsymbol{\xi}^{-1}
$$

and

$$
\overline{\mathcal{S}}=\boldsymbol{\xi} \circ \chi^{-1}
$$

then provide a factorization of $\mathcal{S}: \mathrm{Z} \rightarrow \mathrm{Z}$,

$$
\mathcal{S}=\mathcal{S}^{*} \overline{\mathcal{S}}
$$

The $S L(2, \mathbb{Z})$-representation on the center is thus factored into two representations, generated by $\left(\mathcal{S}^{*}, \mathcal{T}^{*}\right)$ and $(\overline{\mathcal{S}}, \overline{\mathcal{T}})$.

The representation generated by $\left(\mathcal{S}^{*}, \mathcal{T}^{*}\right)$ can be further factored in accordance with the factorization of $\boldsymbol{v}^{*}$ as

$$
\boldsymbol{v}^{*}=\boldsymbol{v}_{(+)}^{*} \boldsymbol{v}_{(-)}^{*}
$$

where

$$
\begin{aligned}
& \boldsymbol{v}_{( \pm)}^{*}=\left\{\begin{array}{l}
\mathbf{1}+\frac{1}{p_{+}} \chi^{\nwarrow \searrow}(1,1) \\
\mathbf{1}+\frac{1}{p_{-}} \chi^{\nwarrow}(1,1)
\end{array}\right. \\
&= \mathbf{1}-\sum_{m=1}^{p_{ \pm}-1} \sum_{a=m-1}^{p_{ \pm}-1}(-1)^{m} \frac{\left(\mathfrak{q}_{ \pm}^{p_{\mp}}-\mathfrak{q}_{ \pm}^{-p_{\mp}}\right)^{2 m-1}}{p_{ \pm}[m]_{ \pm}} \mathfrak{q}_{ \pm}^{p_{\mp} m(m-1-2 a)-p_{\mp}(2+2 a)} \\
& \times\left[\begin{array}{c}
a \\
m-1
\end{array}\right]_{ \pm}^{2} e_{ \pm}^{m} f_{ \pm}^{m} K^{-p_{\mp}(m-2-2 a)}
\end{aligned}
$$

and we calculated $\chi^{\nwarrow \searrow}(1,1)$ and $\chi^{\nwarrow /}(1,1)$ from (4.19) and (4.20) using (B.19) and (B.20). This leads to a further factorization of the $S L(2, \mathbb{Z})$-action on the center, constructed similarly to (5.8)-5.9).

\section{Conclusions}

A remarkable correspondence between modular group actions in nonsemisimple (logarithmic) conformal field theory models and on quantum groups deserves further study. Another quite interesting problem is to extend the category of finite-dimensional $\mathfrak{g}_{p_{+}, p_{-}-}$ rerpesentations such that it becomes equivalent to the category of the $\mathcal{W}_{p_{+}, p_{-}}$algebra representations realized in logarithmic $\left(p_{+}, p_{-}\right)$conformal field theory models. 
Acknowledgments. We are grateful to A. Belavin and S. Parkhomenko for the useful discussions. We thank G. Mutafian for help at the early stages of this work. This paper was supported in part by the RFBR Grant 04-01-00303 and the RFBR-JSPS Grant 0501-02934YaF_a. AMS was also supported in part by the RFBR Grant 05-01-00996. Part of the paper was written when the authors were visiting Kyoto University, and we are grateful to T. Miwa for hospitality.

\section{APPENDIX A. SOME INDECOMPOSABLE $\mathfrak{g}_{p_{+}, p_{-}}$-MODULES}

A.1. $\mathcal{P}_{r, r^{\prime}}^{ \pm, \pm}$modules. As a preparation for the description of projective modules, we introduce modules $\mathcal{P}_{r, r^{\prime}}^{\alpha,+}$ and $\mathcal{P}_{r, r^{\prime}}^{\alpha,-}$, where $\alpha= \pm$, with the respective structure of subquotients described as

(A.1)
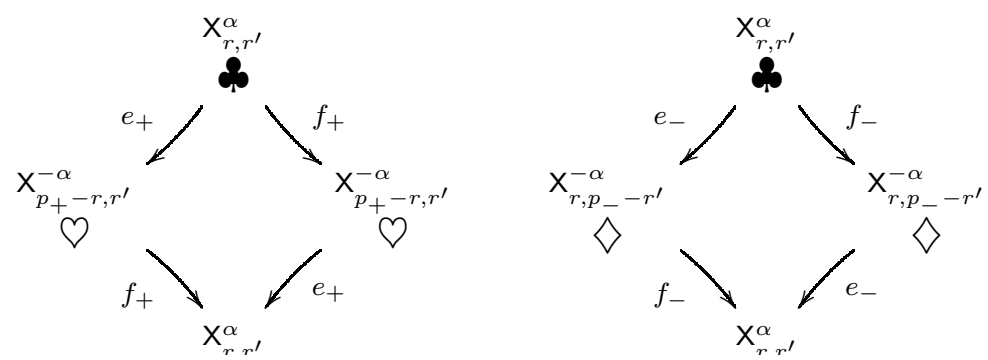

For $1 \leqslant r \leqslant p_{+}-1$ and $1 \leqslant r^{\prime} \leqslant p_{-}$, the $\mathcal{P}_{r, r^{\prime}}^{ \pm,+}$module has the basis

$$
\left\{\mathrm{t}_{n, n^{\prime}}^{\uparrow}, \mathrm{t}_{n, n^{\prime}}^{\downarrow}\right\}_{\substack{0 \leqslant n \leqslant r-1 \\ 0 \leqslant n^{\prime} \leqslant r^{\prime}-1}} \cup\left\{\mathrm{t}_{k, k^{\prime}}^{\overleftarrow{ }}, \mathrm{t}_{k, k^{\prime}}\right\}_{\substack{0 \leqslant k \leqslant p_{+}-r-1 \\ 0 \leqslant k^{\prime} \leqslant r^{\prime}-1}}
$$

where $t_{n, n^{\prime}}^{\uparrow}$ is the basis corresponding to the top $X_{r, r^{\prime}}^{ \pm}$module in (A.1), $t_{n, n^{\prime}}^{\downarrow}$ to the bottom $\mathrm{X}_{r, r^{\prime}}^{ \pm}, \mathrm{t}_{k, k^{\prime}}$ to the right $\mathrm{X}_{p_{+}-r, r^{\prime}}^{\mp}$, and $\mathrm{t}_{k, k^{\prime}}^{\leftarrow}$ to the left $\mathrm{X}_{p_{+}-r, r^{\prime}}^{\mp}$ module. We fix the arbitrariness involved in choosing $\mathrm{t}^{\rightarrow}, \mathrm{t}^{\leftarrow}$, and $\mathrm{t}^{\uparrow}$ by specifying the $\mathfrak{g}_{p_{+}, p_{-}}$-action in $\mathcal{P}_{r, r^{\prime}}^{\alpha,+}$ as

$$
\begin{aligned}
& K \mathrm{t}_{n, n^{\prime}}^{\uparrow}=\alpha \mathfrak{q}_{+}^{r-1-2 n} \mathfrak{q}_{-}^{r^{\prime}-1-2 n^{\prime}} \mathrm{t}_{n, n^{\prime}}^{\uparrow}, \quad K \mathrm{t}_{n, n^{\prime}}^{\downarrow}=\alpha \mathfrak{q}_{+}^{r-1-2 n} \mathfrak{q}_{-}^{r^{\prime}-1-2 n^{\prime}} \mathrm{t}_{n, n^{\prime}}^{\downarrow}, \\
& 0 \leqslant n \leqslant r-1, \quad 0 \leqslant n^{\prime} \leqslant r^{\prime}-1 \text {, }
\end{aligned}
$$

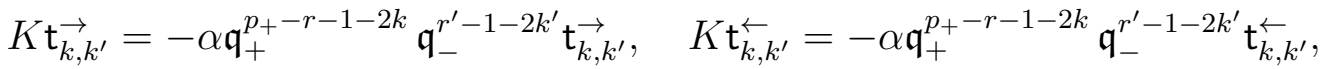

$$
\begin{aligned}
& 0 \leqslant k \leqslant p_{+}-r-1, \quad 0 \leqslant k^{\prime} \leqslant r^{\prime}-1, \\
& e_{+} \mathrm{t}_{n, n^{\prime}}^{\uparrow}= \begin{cases}(\alpha)^{p_{-}}(-1)^{r^{\prime}-1}[n]_{+}[r-n]_{+} \mathrm{t}_{n-1, n^{\prime}}^{\uparrow}+\mathrm{t}_{n-1, n^{\prime}}^{\downarrow}, & 1 \leqslant n \leqslant r-1, \\
\mathrm{t}_{p_{+}-r-1, n^{\prime}}^{\leftarrow}, & n=0,\end{cases} \\
& e_{+} \mathrm{t}_{k, k^{\prime}}= \begin{cases}(-\alpha)^{p_{-}}(-1)^{r^{\prime}-1}[k]_{+}\left[p_{+}-r-k\right]_{+} \mathrm{t}_{k-1, k^{\prime}}, & 1 \leqslant k \leqslant p_{+}-r-1, \\
\mathrm{t}_{r-1, k^{\prime}}^{\downarrow}, & k=0,\end{cases} \\
& \text { (A.3) } e_{+} \mathrm{t}_{k, k^{\prime}}^{\leftarrow}=(-\alpha)^{p_{-}}(-1)^{r^{\prime}-1}[k]_{+}\left[p_{+}-r-k\right]_{+} \mathrm{t}_{k-1, k^{\prime}}^{\leftarrow}, \quad 0 \leqslant k \leqslant p_{+}-r-1 \\
& \text { (with } \mathrm{t}_{-1, k^{\prime}}^{\leftarrow} \equiv 0 \text { ), }
\end{aligned}
$$




$$
\begin{aligned}
& e_{+} \mathrm{t}_{n, n^{\prime}}^{\downarrow}=(\alpha)^{p_{-}}(-1)^{r^{\prime}-1}[n]_{+}[r-n]_{+} \mathrm{t}_{n-1, n^{\prime}}^{\downarrow}, \quad 0 \leqslant n \leqslant r-1 \quad\left(\text { with } \mathrm{t}_{-1, n^{\prime}}^{\downarrow} \equiv 0\right) \text {, } \\
& f_{+} \mathrm{t}_{n, n^{\prime}}^{\uparrow}=\left\{\begin{array}{ll}
\mathrm{t}_{n+1, n^{\prime}}^{\uparrow}, & 0 \leqslant n \leqslant r-2, \\
\mathrm{t}_{0, n^{\prime}}^{\rightarrow}, & n=r-1,
\end{array} \quad f_{+} \mathrm{t}_{k, k^{\prime}}^{\leftarrow}= \begin{cases}\mathrm{t}_{k+1, k^{\prime}}^{\leftarrow}, & 0 \leqslant k \leqslant p_{+}-r-2, \\
\mathrm{t}_{0, k^{\prime}}^{\downarrow}, & k=p_{+}-r-1,\end{cases} \right. \\
& f_{+} \mathrm{t}_{k, k^{\prime}}=\mathrm{t}_{k+1, k^{\prime}}, \quad 0 \leqslant k \leqslant p_{+}-r-1 \quad\left(\text { with } \mathrm{t}_{p_{+}-r, k^{\prime}} \equiv 0\right), \\
& f_{+} \mathrm{t}_{n, n^{\prime}}^{\downarrow}=\mathrm{t}_{n+1, n^{\prime}}^{\downarrow}, \quad 0 \leqslant n \leqslant r-1 \quad\left(\text { with } \mathrm{t}_{r, n^{\prime}}^{\downarrow} \equiv 0\right),
\end{aligned}
$$

where $0 \leqslant n^{\prime}, k^{\prime} \leqslant r^{\prime}-1$, and

$$
\begin{aligned}
& e_{-} \mathrm{t}_{n, n^{\prime}}^{\uparrow}=(\alpha)^{p_{+}}(-1)^{r-1}\left[n^{\prime}\right]_{-}\left[r^{\prime}-n^{\prime}\right]_{-} \mathrm{t}_{n, n^{\prime}-1}^{\uparrow} \quad\left(\text { with } \mathrm{t}_{n,-1}^{\uparrow} \equiv 0\right), \\
& e_{-} \mathrm{t}_{k, k^{\prime}}=(-\alpha)^{p_{+}}(-1)^{p_{+}-r-1}\left[k^{\prime}\right]_{-}\left[r^{\prime}-k^{\prime}\right]_{-} \mathrm{t}_{k, k^{\prime}-1} \quad\left(\text { with } \mathrm{t}_{k,-1} \equiv 0\right) \text {, } \\
& e_{-} \mathrm{t}_{k, k^{\prime}}^{\leftarrow}=(-\alpha)^{p_{+}}(-1)^{p_{+}-r-1}\left[k^{\prime}\right]_{-}\left[r^{\prime}-k^{\prime}\right]_{-} \mathrm{t}_{k, k^{\prime}-1}^{\leftarrow} \quad\left(\text { with } \mathrm{t}_{k,-1}^{\leftarrow} \equiv 0\right), \\
& e_{-} \mathrm{t}_{n, n^{\prime}}^{\downarrow}=(\alpha)^{p_{+}}(-1)^{r-1}\left[n^{\prime}\right]_{-}\left[r^{\prime}-n^{\prime}\right]_{-} \mathrm{t}_{n, n^{\prime}-1}^{\downarrow} \quad\left(\text { with } \mathrm{t}_{n,-1}^{\downarrow} \equiv 0\right) \text {, } \\
& f_{-} \mathrm{t}_{n, n^{\prime}}^{\uparrow}=\mathrm{t}_{n, n^{\prime}+1}^{\uparrow} \quad\left(\text { with } \mathrm{t}_{n, r^{\prime}}^{\uparrow} \equiv 0\right), \quad f_{-} \mathrm{t}_{k, k^{\prime}}=\mathrm{t}_{k, k^{\prime}+1} \quad\left(\text { with } \mathrm{t}_{k, r^{\prime}} \equiv 0\right), \\
& f_{-} \mathrm{t}_{k, k^{\prime}}^{\leftarrow}=\mathrm{t}_{k, k^{\prime}+1}^{\leftarrow} \quad\left(\text { with } \mathrm{t}_{k, r^{\prime}}^{\leftarrow} \equiv 0\right), \quad f_{-} \mathrm{t}_{n, n^{\prime}}^{\downarrow}=\mathrm{t}_{n, n^{\prime}+1}^{\downarrow} \quad\left(\text { with } \mathrm{t}_{n, r^{\prime}}^{\downarrow} \equiv 0\right),
\end{aligned}
$$

where $0 \leqslant n \leqslant r-1,0 \leqslant k \leqslant p_{+}-r-1$, and $0 \leqslant n^{\prime}, k^{\prime} \leqslant r^{\prime}-1$.

For $1 \leqslant r \leqslant p_{+}$and $1 \leqslant r^{\prime} \leqslant p_{-}-1$, we similarly define the $\mathcal{P}_{r, r^{\prime}}^{ \pm,-}$module.

A.2. Projective modules. We describe the projective modules $\mathrm{P}_{r, r^{\prime}}^{ \pm}$for $1 \leqslant r \leqslant p_{+}$and $1 \leqslant r^{\prime} \leqslant p_{-}$. First, for $1 \leqslant r \leqslant p_{+}-1$ and $1 \leqslant r^{\prime} \leqslant p_{-}-1$, the projective module $\mathrm{P}_{r, r^{\prime}}^{ \pm}$is a second extension of the Verma module $\mathrm{V}_{r, r^{\prime}}^{ \pm}$,

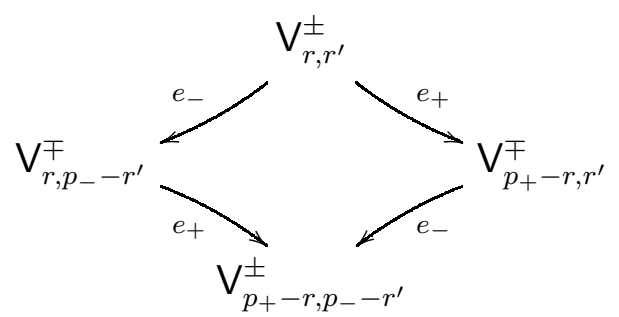

and is therefore a "deck" of sixteen subquotients shown in Fig. 2. We note that $\operatorname{dim} \mathrm{P}_{r, r^{\prime}}^{ \pm}=$ $4 p_{+} p_{-}$for $1 \leqslant r \leqslant p_{+}-1$ and $1 \leqslant r^{\prime} \leqslant p_{-}-1$. Second, $\mathrm{P}_{r, p_{-}}^{ \pm}=\mathcal{P}_{r, p_{-}}^{ \pm,+}$for $1 \leqslant r \leqslant p_{+}-1$ and $\mathrm{P}_{p_{+}, r^{\prime}}^{ \pm}=\mathcal{P}_{p_{+}, r^{\prime}}^{ \pm,-}$for $1 \leqslant r^{\prime} \leqslant p_{-}-1$, where the modules $\mathcal{P}_{r, p_{-}}^{ \pm,+}$and $\mathcal{P}_{p_{+}, r^{\prime}}^{ \pm,-}$are defined above in A.1. Third, $\mathrm{P}_{p_{+}, p_{-}}^{ \pm}=\mathrm{X}_{p_{+}, p_{-}}^{ \pm}$are irreducible.

The module $\mathrm{P}_{r, r^{\prime}}^{ \pm}$has the basis

$$
\mathrm{t}_{n, n^{\prime}}^{\bullet}, \mathrm{b}_{n, n^{\prime}}^{\bullet}, \mathrm{r}_{k, k^{\prime}}^{\bullet}, \mathbf{l}_{k, k^{\prime}}^{\bullet}
$$

where $\bullet \in\{\uparrow, \downarrow, \rightarrow, \leftarrow\}, 0 \leqslant n^{\prime} \leqslant r^{\prime}-1,0 \leqslant k^{\prime} \leqslant p_{-}-r^{\prime}-1,0 \leqslant n, k \leqslant r-1$ if $\bullet \in\{\uparrow, \downarrow\}$, $0 \leqslant n, k \leqslant p_{+}-r-1$ if $\bullet \in\{\rightarrow, \leftarrow\}$, and $\mathrm{t}_{n, n^{\prime}}^{\bullet}$ is the basis corresponding to the top module $\mathcal{P}_{r, r^{\prime}}^{ \pm,+}, \mathrm{b}_{n, n^{\prime}}^{\bullet}$ to the bottom $\mathcal{P}_{r, r^{\prime}}^{ \pm,+}, \mathrm{r}_{k, k^{\prime}}^{\bullet}$ to the right $\mathcal{P}_{r, p_{-}-r^{\prime}}^{\mp,+}$, and $\mathrm{I}_{k, k^{\prime}}^{\bullet}$ to the left module 
$\mathcal{P}_{r, p_{-}-r^{\prime}}^{\mp,+}$ in the diagram

(A.6)

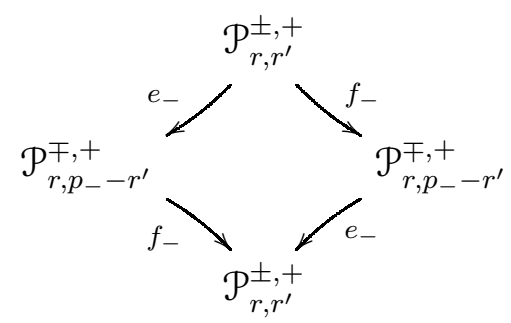

The action of the generators $K, e_{+}$, and $f_{+}$on $\mathrm{t}_{n, n^{\prime}}^{\bullet}$ is defined in $\mathrm{A.3}$ ) and the action of $e_{-}$and $f_{-}$is given by

$$
\begin{aligned}
& e_{-} \mathbf{t}_{n, n^{\prime}}^{\bullet}= \begin{cases}( \pm 1)^{p_{+}}(-1)^{r-1}\left[n^{\prime}\right]_{-}\left[r^{\prime}-n^{\prime}\right]_{-} \mathrm{t}_{n, n^{\prime}-1}^{\bullet}+\mathrm{b}_{n, n^{\prime}-1}^{\bullet}, & 1 \leqslant n^{\prime} \leqslant r^{\prime}-1, \\
\mathbf{l}_{n, p_{-}-r^{\prime}-1}^{\bullet}, & n^{\prime}=0,\end{cases} \\
& f_{-} \mathbf{t}_{n, n^{\prime}}^{\bullet}= \begin{cases}\mathrm{t}_{n, n^{\prime}+1}^{\bullet}, & 0 \leqslant n^{\prime} \leqslant r^{\prime}-2, \\
r_{n, 0}^{\bullet}, & n^{\prime}=r^{\prime}-1 .\end{cases}
\end{aligned}
$$

where $\bullet \in\{\uparrow, \downarrow\}$ and $0 \leqslant n \leqslant r-1$,

$$
\begin{aligned}
& e_{-} \mathrm{t}_{n, n^{\prime}}^{\bullet}= \begin{cases}(\mp 1)^{p_{+}}(-1)^{p_{+}-r-1}\left[n^{\prime}\right]_{-}\left[r^{\prime}-n^{\prime}\right]_{-} \mathrm{t}_{n, n^{\prime}-1}^{\bullet}+\mathrm{b}_{n, n^{\prime}-1}^{\bullet}, & 1 \leqslant n^{\prime} \leqslant r^{\prime}-1, \\
\mathrm{l}_{n, p_{-}-r^{\prime}-1}^{\bullet}, & n^{\prime}=0,\end{cases} \\
& f_{-} \mathrm{t}_{n, n^{\prime}}^{\bullet}= \begin{cases}\mathrm{t}_{n, n^{\prime}+1}^{\bullet}, & 0 \leqslant n^{\prime} \leqslant r^{\prime}-2, \\
\mathrm{r}_{n, 0}^{\bullet}, & n^{\prime}=r^{\prime}-1 .\end{cases}
\end{aligned}
$$

where $\bullet \in\{\rightarrow, \leftarrow\}$ and $0 \leqslant n \leqslant p_{+}-r-1$.

The action of the generators $K, e_{+}, f_{+}$, and $f_{-}$on $r_{n, n^{\prime}}^{\bullet}$ coincides with the action on $\mathcal{P}_{r, p_{-}-r^{\prime}}^{\mp,+}(\operatorname{see} \mathbf{A . 1})$. The action of $e_{-}$is given by

$$
e_{-} \mathbf{r}_{k, k^{\prime}}^{\bullet}= \begin{cases}(\mp 1)^{p_{+}}(-1)^{r-1}\left[k^{\prime}\right]_{-}\left[p_{-}-r^{\prime}-k^{\prime}\right]_{-} \mathbf{r}_{k, k^{\prime}-1}^{\bullet}, & 1 \leqslant k^{\prime} \leqslant p_{-}-r^{\prime}-1, \\ \mathbf{b}_{k, r^{\prime}-1}^{\bullet}, & k^{\prime}=0,\end{cases}
$$

where $\bullet \in\{\uparrow, \downarrow\}$ and $0 \leqslant k \leqslant r-1$,

$$
e_{-} \mathbf{r}_{k, k^{\prime}}^{\bullet}= \begin{cases}( \pm 1)^{p_{+}}(-1)^{p_{+}-r-1}\left[k^{\prime}\right]_{-}\left[p_{-}-r^{\prime}-k^{\prime}\right]_{-} \mathbf{r}_{k, k^{\prime}-1}^{\bullet}, & 1 \leqslant k^{\prime} \leqslant p_{-}-r^{\prime}-1, \\ \mathbf{b}_{k, r^{\prime}-1}^{\bullet}, & k^{\prime}=0,\end{cases}
$$

where $\bullet \in\{\rightarrow, \leftarrow\}$ and $0 \leqslant k \leqslant p_{+}-r-1$.

The action of the generators $K, e_{+}, f_{+}$, and $e_{-}$on $\mathrm{I}_{n, n^{\prime}}^{\bullet}$ coincides with the action on $\mathcal{P}_{r, p_{-}-r^{\prime}}^{\mp,+}($ see $\mathbf{A . 1})$. The action of $f_{-}$is given by

$$
\left.f_{-}\right|_{k, k^{\prime}} ^{\bullet}= \begin{cases}\left.\right|_{k, k^{\prime}+1} ^{\bullet}, & 0 \leqslant k^{\prime} \leqslant p_{-}-r^{\prime}-2 \\ \mathrm{~b}_{k, 0}^{\bullet}, & k^{\prime}=p_{-}-r^{\prime}-1\end{cases}
$$


where $\bullet \in\{\uparrow, \downarrow\}$ and $0 \leqslant k \leqslant r-1$,

$$
\left.f_{-}\right|_{k, k^{\prime}} ^{\bullet}= \begin{cases}\mathbf{l}_{k, k^{\prime}+1}^{\bullet}, & 0 \leqslant k^{\prime} \leqslant p_{-}-r^{\prime}-2, \\ \mathbf{b}_{k, 0}^{\bullet}, & k^{\prime}=p_{-}-r^{\prime}-1,\end{cases}
$$

where $\bullet \in\{\rightarrow, \leftarrow\}$ and $0 \leqslant k \leqslant p_{+}-r-1$.

The $\mathfrak{g}_{p_{+}, p_{-}}$-action on $b_{n, n^{\prime}}^{\bullet}$ is defined in A.1.

\section{APPENDiX B. THE CENTER OF $\mathfrak{g}_{p_{+}, p_{-}}$}

Here, we find the center of $\mathfrak{g}_{p_{+}, p_{-}}$using the isomorphism between the center and the algebra of bimodule endomorphisms of the regular representation. In B.1 we study decomposition of the regular representation as a $\mathfrak{g}_{p_{+}, p_{-}}$-bimodule. In B.2 we describe the algebra of bimodule endomorphisms of the regular representation. In $\mathbf{B . 3}$, the canonical central elements are also constructed explicitly in terms of the algebra generators. $\operatorname{In} \mathbf{B . 4}$, we collect several calculations needed in the proofs in this paper.

B.1. Decomposition of the regular representation. We study the decomposition of the regular representation as a $\mathfrak{g}_{p_{+}, p_{-}}$-bimodule. For this, we closely follow the strategy proposed in [5] for $(p, 1)$ models. The starting point is to introduce indecomposable $\mathfrak{g}_{p_{+}, p_{-}}$-bimodules $Q_{r, r^{\prime}}^{ \pm, \pm}$defined as follows. For $1 \leqslant r \leqslant p_{+}-1$ and $1 \leqslant r^{\prime} \leqslant p_{-}, Q_{r, r^{\prime}}^{ \pm,+}$is composed of eight subquotients with the left $\mathfrak{g}_{p_{+}, p_{-}}$-action represented as

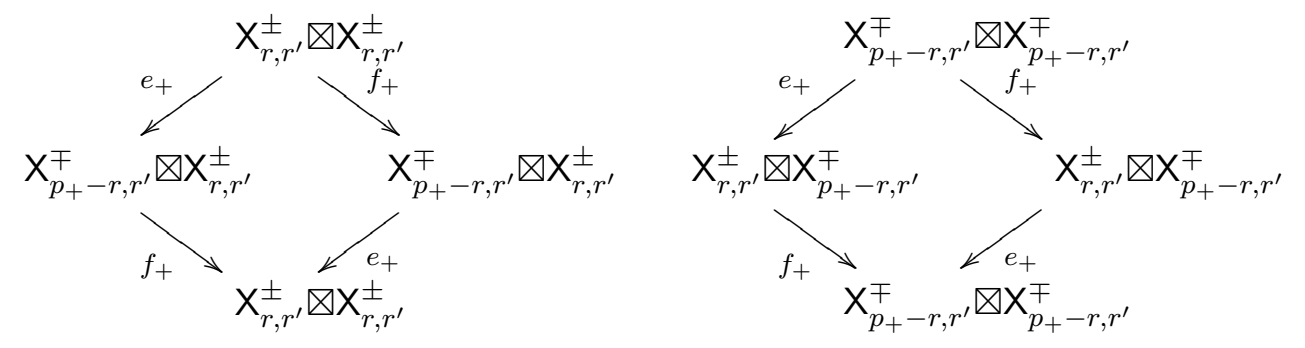

and the right action as

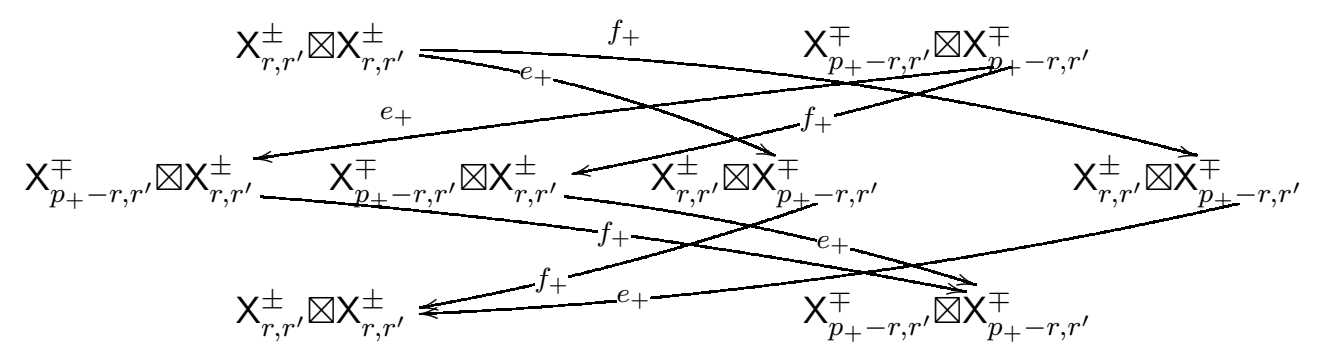

In these diagrams, as we have noted, it is understood that the $\mathfrak{g}_{p_{+}, p_{-}}$-action on each subquotient is changed compared with the action on the corresponding irreducible representa- 
tion in agreement with the arrows connecting a given subquotient with others. For example, $e_{+}$maps the highest-weight vector of $\mathrm{X}_{r, r^{\prime}}^{+}$into the lowest-weight vector in $\mathrm{X}_{p_{+}-r, r^{\prime}}^{-}$.

For $1 \leqslant r \leqslant p_{+}$and $1 \leqslant r^{\prime} \leqslant p_{-}-1$, the bimodules $Q_{r, r^{\prime}}^{ \pm,-}$are defined in the same way with the substitution $e_{+} \mapsto e_{-}, f_{+} \mapsto f_{-}$, and $\mathrm{X}_{p_{+}-r, r^{\prime}}^{\mp} \mapsto \mathrm{X}_{r, p_{-}-r^{\prime}}^{\mp}$ in the above diagrams.

B.1.1. Proposition. As a $\mathfrak{g}_{p_{+}, p_{-}}$-bimodule, the regular representation decomposes as where

$$
\operatorname{Reg}=\bigoplus_{\left(r, r^{\prime}\right) \in \mathcal{I}_{1}} \mathcal{Q}\left(r, r^{\prime}\right) \oplus \bigoplus_{r=1}^{p_{+}-1} \mathcal{Q}\left(r, p_{-}\right) \oplus \bigoplus_{r^{\prime}=1}^{p_{-}-1} \mathcal{Q}\left(p_{+}, r^{\prime}\right) \oplus \mathcal{L}\left(p_{+}, p_{-}\right) \oplus \mathcal{L}\left(0, p_{-}\right) \text {, }
$$

(1) $\mathcal{Q}\left(p_{+}, p_{-}\right)=\mathrm{X}_{p_{+}, p_{-}}^{+} \otimes \mathrm{X}_{p_{+}, p_{-}}^{+}$and $Q\left(0, p_{-}\right)=\mathrm{X}_{p_{+}, p_{-}}^{-} \otimes \mathrm{X}_{p_{+}, p_{-}}^{-}$are simple bimodules;

(2) $\mathcal{Q}\left(r, p_{-}\right)=Q_{r, p_{-}}^{+,+}$and $\mathcal{Q}\left(p_{+}, r^{\prime}\right)=Q_{p_{+}, r^{\prime}}^{+,-}$for $1 \leqslant r \leqslant p_{+}-1$ and $1 \leqslant r^{\prime} \leqslant p_{-}-1$;

(3) the bimodules $Q\left(r, r^{\prime}\right), 1 \leqslant r \leqslant p_{+}-1$ and $1 \leqslant r^{\prime} \leqslant p_{-}-1$, are indecomposable and admit two length-3 filtrations:

$$
0 \subset \mathcal{R}_{2}^{+}\left(r, r^{\prime}\right) \subset \mathcal{R}^{+}\left(r, r^{\prime}\right) \subset \mathcal{Q}\left(r, r^{\prime}\right),
$$

where the structure of subquotients is given by

$$
\begin{aligned}
\mathcal{Q}\left(r, r^{\prime}\right) / \mathcal{R}^{+}\left(r, r^{\prime}\right) & =Q_{r, r^{\prime}}^{+,+} \oplus \mathcal{Q}_{r, p_{-}-r^{\prime}}^{-,+}, \\
\mathcal{R}^{+}\left(r, r^{\prime}\right) / \mathcal{R}_{2}^{+}\left(r, r^{\prime}\right) & =2 Q_{r, p_{-}-r^{\prime}}^{-,+} \oplus 2 Q_{r, r^{\prime}}^{+,+}, \\
\mathcal{R}_{2}^{+}\left(r, r^{\prime}\right) & =Q_{r, r^{\prime}}^{+,+} \oplus Q_{r, p_{-}-r^{\prime}}^{-,+},
\end{aligned}
$$

and

$$
0 \subset \mathcal{R}_{2}^{-}\left(r, r^{\prime}\right) \subset \mathcal{R}^{-}\left(r, r^{\prime}\right) \subset \mathcal{Q}\left(r, r^{\prime}\right),
$$

where the structure of subquotients is given by

$$
\begin{aligned}
\mathcal{Q}\left(r, r^{\prime}\right) / \mathcal{R}^{-}\left(r, r^{\prime}\right) & =Q_{r, r^{\prime}}^{+,-} \oplus \mathcal{Q}_{p_{+}-r, r^{\prime}}^{-,-}, \\
\mathcal{R}^{-}\left(r, r^{\prime}\right) / \mathcal{R}_{2}^{-}\left(r, r^{\prime}\right) & =2 Q_{p_{+}-r, r^{\prime}}^{-,-} \oplus 2 Q_{r, r^{\prime}}^{+,-}, \\
\mathcal{R}_{2}^{-}\left(r, r^{\prime}\right) & =Q_{r, r^{\prime}}^{+,-} \oplus \mathcal{Q}_{p_{+}-r, r^{\prime}}^{-,-}
\end{aligned}
$$

We do not repeat the standard steps leading to this statement, the derivation is totally similar to the one in [5].

B.2. Bimodule homomorphisms and the center. Here, we study the algebra of bimodule endomorphisms of the regular representation Reg. Simultaneously, we derive the structure of the $\mathfrak{g}_{p_{+}, p_{-}}$center because bimodule endomorphisms of the regular representation are in a one-to-one correspondence with elements in the center. 
B.2.1. Clearly, the algebra of bimodule endomorphisms of Reg decomposes, in agreement with (2.21), as

$$
\begin{aligned}
\operatorname{End}(\operatorname{Reg})= & \bigoplus_{\left(r, r^{\prime}\right) \in \mathcal{I}_{1}} \operatorname{End}\left(\mathcal{Q}\left(r, r^{\prime}\right)\right) \oplus \bigoplus_{r=1}^{p_{+}-1} \operatorname{End}\left(\mathcal{Q}\left(r, p_{-}\right)\right) \oplus \\
& \bigoplus_{r^{\prime}=1}^{p_{-}-1} \operatorname{End}\left(\mathcal{Q}\left(p_{+}, r^{\prime}\right)\right) \oplus \operatorname{End}\left(\mathcal{Q}\left(p_{+}, p_{-}\right)\right) \oplus \operatorname{End}\left(\mathcal{Q}\left(0, p_{-}\right)\right) .
\end{aligned}
$$

For each $Q\left(r, r^{\prime}\right)$, there is a bimodule endomorphism $\boldsymbol{e}\left(r, r^{\prime}\right):$ Reg $\rightarrow$ Reg that acts as identity on $\mathcal{Q}\left(r, r^{\prime}\right)$ and is zero on $\mathcal{Q}\left(s, s^{\prime}\right)$ unless $s=r$ and $s^{\prime}=r^{\prime}$. These endomorphisms give rise to $\frac{1}{2}\left(p_{+}+1\right)\left(p_{-}+1\right)$ primitive idempotents (also denoted by $\boldsymbol{e}\left(r, r^{\prime}\right)$ ) in the center of $\mathfrak{g}_{p_{+}, p_{-}}$.

Next, for each $\mathcal{Q}\left(r, r^{\prime}\right)$ with $\left(r, r^{\prime}\right) \in \mathcal{I}_{1}$,

(1) there is a homomorphism $\boldsymbol{v}^{\nearrow}\left(r, r^{\prime}\right): \mathcal{Q}\left(r, r^{\prime}\right) \rightarrow \mathcal{Q}\left(r, r^{\prime}\right)$ (defined up to a nonzero factor) whose image is $Q_{r, r^{\prime}}^{+,+}$; in other words, $\boldsymbol{v}^{\nearrow}\left(r, r^{\prime}\right)$ sends the quotient $Q_{r, r^{\prime}}^{+,+}$ (see (B.1)) into the subbimodule $\mathcal{Q}_{r, r^{\prime}}^{+,+}$at the bottom of $\mathcal{Q}\left(r, r^{\prime}\right)$ and is zero on $Q\left(s, s^{\prime}\right)$ unless $s=r$ and $s^{\prime}=r^{\prime}$.

(2) Similarly, there is a central element associated with the homomorphism $\boldsymbol{v}^{\nwarrow}\left(r, r^{\prime}\right)$ : $\mathcal{Q}\left(r, r^{\prime}\right) \rightarrow \mathcal{Q}\left(r, r^{\prime}\right)$ with the image $\mathcal{Q}_{r, r^{\prime}}^{+,-}$, i.e., sending the quotient $\mathcal{Q}_{r, r^{\prime}}^{+,-}(\operatorname{see}(\underline{B}$. 2$)$ ) into the subbimodule $\mathcal{Q}_{r, r^{\prime}}^{+,-}$(and zero on $\mathcal{Q}\left(s, s^{\prime}\right)$ unless $s=r$ and $s^{\prime}=r^{\prime}$ ).

(3) A central element is associated with the homomorphism $\boldsymbol{v}^{<}\left(r, r^{\prime}\right): \mathcal{Q}\left(r, r^{\prime}\right) \rightarrow$ $\mathcal{Q}\left(r, r^{\prime}\right)$ with the image $Q_{r, p_{-} r^{\prime}}^{-,+}$, i.e., sending the quotient $Q_{r, p_{-} r^{\prime}}^{-,+}($see $(\overline{B .1})$ ) into the subbimodule $Q_{r, p_{-}-r^{\prime}}^{-,+}\left(\right.$and zero on $\mathcal{Q}\left(s, s^{\prime}\right)$ unless $s=r$ and $\left.s^{\prime}=r^{\prime}\right)$.

(4) Similarly, there is a central element associated with the homomorphism $\boldsymbol{v}^{\searrow}\left(r, r^{\prime}\right)$ : $\mathcal{Q}\left(r, r^{\prime}\right) \rightarrow \mathcal{Q}\left(r, r^{\prime}\right)$ with the image $\mathcal{Q}_{p_{+}-r, r^{\prime}}^{-,-}$, i.e., sending the quotient $\mathcal{Q}_{p_{+}-r, r^{\prime}}^{-,-}$ (see $(\underline{\mathrm{B} .2})$ ) into the subbimodule $\mathcal{Q}_{p_{+}-r, r^{\prime}}^{-,-}$(and zero on $\mathcal{Q}\left(s, s^{\prime}\right)$ unless $s=r$ and $\left.s^{\prime}=r^{\prime}\right)$.

(5) There is also a homomorphism $\boldsymbol{w}^{\uparrow}\left(r, r^{\prime}\right): \mathcal{Q}\left(r, r^{\prime}\right) \rightarrow \mathcal{Q}\left(r, r^{\prime}\right)$ (defined up to a nonzero factor) whose image is $\mathbf{X}_{r, r^{\prime}}^{+} \otimes \mathbf{X}_{r, r^{\prime}}^{+}$; in other words, $\boldsymbol{w}^{\uparrow}\left(r, r^{\prime}\right)$ sends the quotient $\mathrm{X}_{r, r^{\prime}}^{+} \otimes \mathrm{X}_{r, r^{\prime}}^{+}$into the subbimodule $\mathrm{X}_{r, r^{\prime}}^{+} \otimes \mathrm{X}_{r, r^{\prime}}^{+}$at the bottom of $\mathcal{Q}\left(r, r^{\prime}\right)$ and is zero on $\mathcal{Q}\left(s, s^{\prime}\right)$ unless $s=r$ and $s^{\prime}=r^{\prime}$.

(6) Similarly, there is a homomorphism $\boldsymbol{w}^{\rightarrow}\left(r, r^{\prime}\right): \mathcal{Q}\left(r, r^{\prime}\right) \rightarrow \mathcal{Q}\left(r, r^{\prime}\right)$ whose image is $\mathrm{X}_{p_{+}-r, r^{\prime}}^{-} \otimes \mathrm{X}_{p_{+}-r, r^{\prime}}^{-}$; in other words, $\boldsymbol{w}^{\rightarrow}\left(r, r^{\prime}\right)$ sends the quotient $\mathrm{X}_{p_{+}-r, r^{\prime}}^{-} \otimes$ $\mathrm{X}_{p_{+}-r, r^{\prime}}^{-}$into the subbimodule $\mathrm{X}_{p_{+}-r, r^{\prime}}^{-} \otimes \mathrm{X}_{p_{+}-r, r^{\prime}}^{-}$at the bottom of $\mathcal{Q}\left(r, r^{\prime}\right)$ and is zero on $\mathcal{Q}\left(s, s^{\prime}\right)$ unless $s=r$ and $s^{\prime}=r^{\prime}$.

(7) There is also a homomorphism $\boldsymbol{w}^{\leftarrow}\left(r, r^{\prime}\right): \mathcal{Q}\left(r, r^{\prime}\right) \rightarrow \mathcal{Q}\left(r, r^{\prime}\right)$ whose image is $\mathrm{X}_{r, p_{-} r^{\prime}}^{-} \otimes \mathrm{X}_{r, p_{-} r^{\prime}}^{-} ;$in other words, $\boldsymbol{w}^{\leftarrow}\left(r, r^{\prime}\right)$ sends the quotient $\mathbf{X}_{r, p_{-}-r^{\prime}}^{-} \otimes \mathbf{X}_{r, p_{-}-r^{\prime}}^{-}$ into the subbimodule $\mathbf{X}_{r, p_{-}-r^{\prime}}^{-} \otimes \mathbf{X}_{r, p_{-}-r^{\prime}}^{-}$at the bottom of $\mathcal{Q}\left(r, r^{\prime}\right)$ and is zero on $\mathcal{Q}\left(s, s^{\prime}\right)$ unless $s=r$ and $s^{\prime}=r^{\prime}$. 
(8) Similarly, there is also a homomorphism $\boldsymbol{w}^{\downarrow}\left(r, r^{\prime}\right): \mathcal{Q}\left(r, r^{\prime}\right) \rightarrow \mathcal{Q}\left(r, r^{\prime}\right)$ whose image is $\mathrm{X}_{p_{+}-r, p_{-}-r^{\prime}}^{+} \otimes \mathrm{X}_{p_{+}-r, p_{-} r^{\prime}}^{+}$; in other words, $\boldsymbol{w}^{\downarrow}\left(r, r^{\prime}\right)$ sends the quotient $\mathrm{X}_{p_{+}-r, p_{-} r^{\prime}}^{+} \otimes \mathrm{X}_{p_{+-}, p_{-} r^{\prime}}^{+}$into the subbimodule $\mathrm{X}_{p_{+}-r, p_{-}-r^{\prime}}^{+} \otimes \mathrm{X}_{p_{+}-r, p_{-}-r^{\prime}}^{+}$at the bottom of $\mathcal{Q}\left(r, r^{\prime}\right)$ and is zero on $\mathcal{Q}\left(s, s^{\prime}\right)$ unless $s=r$ and $s^{\prime}=r^{\prime}$.

These maps give $4\left(p_{+}-1\right)\left(p_{-}-1\right)$ nilpotent elements $\boldsymbol{w}^{\leftarrow}\left(r, r^{\prime}\right), \boldsymbol{w}^{\uparrow}\left(r, r^{\prime}\right), \boldsymbol{w}^{\rightarrow}\left(r, r^{\prime}\right)$, $\boldsymbol{w}^{\downarrow}\left(r, r^{\prime}\right), \boldsymbol{v}^{\nwarrow}\left(r, r^{\prime}\right), \boldsymbol{v}^{\nearrow}\left(r, r^{\prime}\right), \boldsymbol{v}^{\searrow}\left(r, r^{\prime}\right), \boldsymbol{v}^{\swarrow}\left(r, r^{\prime}\right)$. From the structure of the maps, it is obvious that relations (2.22) are satisfied and that all other compositions are zero.

Next, for each $\mathcal{Q}\left(r, p_{-}\right)$with $1 \leqslant r \leqslant p_{+}-1$, there is a homomorphism $\boldsymbol{v}^{\uparrow}\left(r, p_{-}\right)$: $\mathcal{Q}\left(r, p_{-}\right) \rightarrow \mathcal{Q}\left(r, p_{-}\right)$with the image $\mathrm{X}_{r, p_{-}}^{+} \otimes \mathrm{X}_{r, p_{-}}^{+}$; in other words, $\boldsymbol{v}^{\uparrow}\left(r, p_{-}\right)$sends the quotient $\mathrm{X}_{r, p_{-}}^{+} \otimes \mathrm{X}_{r, p_{-}}^{+}$into the subbimodule $\mathrm{X}_{r, p_{-}}^{+} \otimes \mathrm{X}_{r, p_{-}}^{+}$at the bottom of $\mathcal{Q}\left(r, p_{-}\right)$ and is zero on $\mathcal{Q}\left(r^{\prime}, p_{-}\right)$with $r^{\prime} \neq r$. Similarly, for each $r=1, \ldots, p_{+}-1$, there is a central element associated with the homomorphism $\boldsymbol{v}^{\rightarrow}\left(r, p_{-}\right): \mathcal{Q}\left(r, p_{-}\right) \rightarrow \mathcal{Q}\left(r, p_{-}\right)$ with the image $\mathbf{X}_{p_{+}-r, p_{-}}^{-} \otimes \mathbf{X}_{p_{+}-r, p_{-}}^{-}$, i.e., sending the quotient $\mathbf{X}_{p_{+}-r, p_{-}}^{-} \otimes \mathbf{X}_{p_{+}-r, p_{-}}^{-}$into the subbimodule $\mathbf{X}_{p_{+}-r, p_{-}}^{-} \otimes \mathbf{X}_{p_{+}-r, p_{-}}^{-}$(and zero on $\mathcal{Q}\left(r^{\prime}, p_{-}\right)$with $r^{\prime} \neq r$ ). Next, for each $\mathcal{Q}\left(p_{+}, s\right)$ with $1 \leqslant s \leqslant p_{-}-1$, there are similar homomorphisms $\boldsymbol{v}^{\uparrow}\left(p_{+}, s\right)$ and $\boldsymbol{v}^{\leftarrow}\left(p_{+}, s\right): \mathcal{Q}\left(p_{+}, s\right) \rightarrow \mathcal{Q}\left(p_{+}, s\right)$.

This gives $2\left(p_{+}-1\right)+2\left(p_{-}-1\right)$ more elements $\boldsymbol{v}^{\uparrow}\left(r, p_{-}\right), \boldsymbol{v}^{\rightarrow}\left(r, p_{-}\right), \boldsymbol{v}^{\uparrow}\left(p_{+}, s\right)$, $\boldsymbol{v}^{\leftarrow}\left(p_{+}, s\right)$, which are obviously in the radical of the center.

The above is just restated in 2.4.1

B.2.2. Canonical decomposition of central elements. Any central element $A$ can be decomposed in the above central elements as

$$
\begin{aligned}
A= & \sum_{\left(r, r^{\prime}\right) \in \mathcal{I}} a_{r, r^{\prime}} \boldsymbol{e}\left(r, r^{\prime}\right)+\sum_{\substack{\left(r, r^{\prime}\right) \in \mathcal{I}_{1} \\
\bullet \in\{\gamma, \nwarrow, \searrow, \swarrow\}}} b_{r, r^{\prime}}^{\bullet} \boldsymbol{v}^{\bullet}\left(r, r^{\prime}\right) \\
& +\sum_{\substack{\left(r, r^{\prime}\right) \in \mathcal{I}_{1} \\
\bullet \in\{\uparrow, \downarrow, \rightarrow, \leftarrow\}}} c_{r, r^{\prime}}^{\bullet} \boldsymbol{w}^{\bullet}\left(r, r^{\prime}\right)+\sum_{\substack{r=1 \\
\bullet \in\{\uparrow, \rightarrow\}}}^{p_{+}-1} b_{r}^{\bullet} \boldsymbol{v}^{\bullet}\left(r, p_{-}\right)+\sum_{\substack{r^{\prime}=1 \\
\bullet \in\{\uparrow, \leftarrow\}}}^{p_{-}-1} c_{r^{\prime}}^{\bullet} \boldsymbol{v}^{\bullet}\left(p_{+}, r^{\prime}\right) .
\end{aligned}
$$

It immediately follows from B.2.1 that the coefficient $a_{r, r^{\prime}}$ is the eigenvalue of $A$ in the irreducible representation $\mathrm{X}_{r, r^{\prime}}^{+}$. To determine the $b_{r, r^{\prime}}^{\bullet}, c_{r, r^{\prime}}^{\bullet}, b_{r}^{\bullet}$, and $c_{r^{\prime}}^{\bullet}$ coefficients similarly, we fix the normalizations such that in terms of the respective bases described in $\mathbf{A . 2}$, $\boldsymbol{v}^{\bullet}\left(r, r^{\prime}\right), \boldsymbol{w}^{\bullet}\left(r, r^{\prime}\right), \boldsymbol{v}^{\bullet}\left(r, p_{-}\right)$, and $\boldsymbol{v}^{\bullet}\left(p_{+}, r^{\prime}\right)$ act as

$$
\boldsymbol{v}^{\nearrow}\left(r, r^{\prime}\right) \mathrm{t}_{n, n^{\prime}}^{\bullet}=\mathrm{b}_{n, n^{\prime}}^{\bullet}, \bullet \in\{\uparrow, \downarrow, \rightarrow, \leftarrow\}, \quad \text { in } \mathrm{P}_{r, r^{\prime}}^{+} \text {and } \mathrm{P}_{p_{+}-r, r^{\prime}}^{-},
$$

with $\boldsymbol{v}^{\nearrow}\left(r, r^{\prime}\right)$ being identically zero on any module other than $\mathrm{P}_{r, r^{\prime}}^{+}$or $\mathrm{P}_{p_{+}-r, r^{\prime}}^{-}$; similarly,

$$
\begin{aligned}
& \boldsymbol{v}^{\nwarrow}\left(r, r^{\prime}\right) \mathrm{x}_{n, n^{\prime}}^{\uparrow}=\mathrm{x}_{n, n^{\prime}}^{\downarrow}, \quad \mathrm{x} \in\{\mathrm{t}, \mathrm{r}, \mathrm{l}, \mathrm{b}\}, \quad \text { in } \mathrm{P}_{r, r^{\prime}}^{+} \text {and } \mathrm{P}_{r, p_{-}-r^{\prime}}^{-}, \\
& \boldsymbol{v}^{\swarrow}\left(r, r^{\prime}\right) \mathrm{t}_{n, n^{\prime}}^{\bullet}=\mathrm{b}_{n, n^{\prime}}^{\bullet}, \quad \bullet \in\{\uparrow, \downarrow, \rightarrow, \leftarrow\}, \quad \text { in } \mathrm{P}_{r, p_{-}-r^{\prime}}^{-} \text {and } \mathrm{P}_{p_{+}-r, p_{-}-r^{\prime}}^{+},
\end{aligned}
$$




$$
\begin{aligned}
& \boldsymbol{v}^{\searrow}\left(r, r^{\prime}\right) \mathrm{x}_{n, n^{\prime}}^{\uparrow}=\mathrm{x}_{n, n^{\prime}}^{\downarrow}, \mathrm{x} \in\{\mathrm{t}, \mathrm{r}, \mathrm{l}, \mathrm{b}\}, \quad \text { in } \mathrm{P}_{p_{+}-r, r^{\prime}}^{-} \text {and } \mathrm{P}_{p_{+}-r, p_{-}-r^{\prime}}^{+}, \\
& \boldsymbol{w}^{\uparrow}\left(r, r^{\prime}\right) \mathrm{t}_{n, n^{\prime}}^{\uparrow}=\mathrm{b}_{n, n^{\prime}}^{\downarrow} \quad \text { in } \mathrm{P}_{r, r^{\prime}}^{+}, \\
& \boldsymbol{w}^{\rightarrow}\left(r, r^{\prime}\right) \mathrm{t}_{n, n^{\prime}}^{\uparrow}=\mathrm{b}_{n, n^{\prime}}^{\downarrow} \quad \text { in } \mathrm{P}_{p_{+}-r, r^{\prime}}^{-}, \\
& \boldsymbol{w}^{\leftarrow}\left(r, r^{\prime}\right) \mathrm{t}_{n, n^{\prime}}^{\uparrow}=\mathrm{b}_{n, n^{\prime}}^{\downarrow} \text { in } \mathrm{P}_{r, p_{-}-r^{\prime}}^{-}, \\
& \boldsymbol{w}^{\downarrow}\left(r, r^{\prime}\right) \mathrm{t}_{n, n^{\prime}}^{\uparrow}=\mathrm{b}_{n, n^{\prime}}^{\downarrow} \text { in } \mathrm{P}_{p_{+}-r, p_{-}-r^{\prime}}^{+}, \\
& \boldsymbol{v}^{\uparrow}\left(r, p_{-}\right) \mathrm{t}_{n, n^{\prime}}^{\uparrow}=\mathrm{t}_{n, n^{\prime}}^{\downarrow} \text { in } \mathrm{P}_{r, p_{-}}^{+}, \\
& \boldsymbol{v}^{\rightarrow}\left(r, p_{-}\right) \mathrm{t}_{n, n^{\prime}}^{\uparrow}=\mathrm{t}_{n, n^{\prime}}^{\downarrow} \text { in } \mathrm{P}_{p_{+}-r, p_{-}}^{-}, \\
& \boldsymbol{v}^{\uparrow}\left(p_{+}, r^{\prime}\right) \mathrm{t}_{n, n^{\prime}}^{\uparrow}=\mathrm{t}_{n, n^{\prime}}^{\downarrow} \text { in } \mathrm{P}_{p_{+}, r^{\prime}}^{+}, \\
& \boldsymbol{v}^{\leftarrow}\left(p_{+}, r^{\prime}\right) \mathrm{t}_{n, n^{\prime}}^{\uparrow}=\mathrm{t}_{n, n^{\prime}}^{\downarrow} \text { in } \mathrm{P}_{p_{+}, p_{-}-r^{\prime}}^{-}
\end{aligned}
$$

Then the coefficients in $(\mathrm{B} .3)$ are determined from the relation

$$
\begin{aligned}
& A \mathrm{t}_{n, n^{\prime}}^{\bullet}=b_{r, r^{\prime}}^{\nearrow} \mathbf{b}_{n, n^{\prime}}^{\bullet} \quad \text { in } \quad \mathrm{P}_{r, r^{\prime}}^{+}, \quad \bullet \in\{\uparrow, \downarrow, \rightarrow, \leftarrow\}, \\
& A \mathrm{x}_{n, n^{\prime}}^{\uparrow}=b_{r, r^{\prime}}^{\nwarrow} \mathrm{x}_{n, n^{\prime}}^{\downarrow} \quad \text { in } \quad \mathrm{P}_{r, p_{-}-r^{\prime}}^{-}, \quad \mathrm{x} \in\{\mathrm{t}, \mathrm{r}, \mathrm{l}, \mathrm{b}\}, \\
& A \mathrm{t}_{n, n^{\prime}}^{\bullet}=b_{r, r^{\prime}}^{\swarrow} \mathrm{b}_{n, n^{\prime}}^{\bullet} \quad \text { in } \quad \mathrm{P}_{p_{+}-r, p_{-}-r^{\prime}}^{+}, \quad \bullet \in\{\uparrow, \downarrow, \rightarrow, \leftarrow\} \text {, } \\
& A \mathrm{x}_{n, n^{\prime}}^{\uparrow}=b_{r, r^{\prime}}^{\searrow} \mathrm{x}_{n, n^{\prime}}^{\downarrow} \quad \text { in } \quad \mathrm{P}_{p_{+}-r, r^{\prime}}^{-}, \quad \mathrm{x} \in\{\mathrm{t}, \mathrm{r}, \mathrm{l}, \mathrm{b}\}, \\
& A \mathrm{t}_{n, n^{\prime}}^{\uparrow}=c_{r, r^{\prime}}^{\uparrow} \mathrm{b}_{n, n^{\prime}}^{\downarrow} \quad \text { in } \quad \mathrm{P}_{r, r^{\prime}}^{+} \text {, } \\
& A \mathrm{t}_{n, n^{\prime}}^{\uparrow}=\overrightarrow{c_{r, r^{\prime}}} \mathrm{b}_{n, n^{\prime}}^{\downarrow} \quad \text { in } \quad \mathrm{P}_{p_{+}-r, r^{\prime}}^{-} \text {, } \\
& A \mathrm{t}_{n, n^{\prime}}^{\uparrow}=c_{r, r^{\prime}}^{\leftarrow} \mathrm{b}_{n, n^{\prime}}^{\downarrow} \quad \text { in } \quad \mathrm{P}_{r, p_{-}-r^{\prime}}^{-} \\
& A \mathrm{t}_{n, n^{\prime}}^{\uparrow}=c_{r, r^{\prime}}^{\downarrow} \mathrm{b}_{n, n^{\prime}}^{\downarrow} \quad \text { in } \quad \mathrm{P}_{p_{+}-r, p_{-}-r^{\prime}}^{+} \text {, } \\
& A \mathrm{t}_{n, n^{\prime}}^{\uparrow}=b_{r}^{\uparrow} \mathrm{t}_{n, n^{\prime}}^{\downarrow} \quad \text { in } \quad \mathrm{P}_{r, p_{-}}^{+} \text {, } \\
& A \mathrm{t}_{n, n^{\prime}}^{\uparrow}=b_{r} \rightarrow \mathrm{t}_{n, n^{\prime}}^{\downarrow} \quad \text { in } \quad \mathrm{P}_{p_{+}-r, p_{-}}^{-} \text {, } \\
& A \mathrm{t}_{n, n^{\prime}}^{\uparrow}=c_{r^{\prime}}^{\uparrow} t_{n, n^{\prime}}^{\downarrow} \quad \text { in } \quad \mathrm{P}_{p_{+}, r^{\prime}}^{-}, \\
& A \mathrm{t}_{n, n^{\prime}}^{\uparrow}=c_{r^{\prime}}^{\leftarrow} \mathrm{t}_{n, n^{\prime}}^{\downarrow} \quad \text { in } \quad \mathrm{P}_{p_{+}, p_{-}-r^{\prime}}^{-}
\end{aligned}
$$

These formulas are used many times in explicit calculations in the text.

B.3. Explicit construction of the canonical basis in the center. To explicitly construct the canonical central elements in 2.4.1 in terms of the $\mathfrak{g}_{p_{+}, p_{-}}$generators, we proceed similarly to [5, 19].

Let $\left(r, r^{\prime}\right) \in \mathcal{I}($ see (1.12) $)$. We set

$$
\begin{aligned}
& \beta_{+}\left(r, r^{\prime}\right)=(-1)^{r^{\prime}}\left(\mathfrak{q}_{+}^{p_{-} r}+\mathfrak{q}_{+}^{-p_{-} r}\right), \\
& \beta_{-}\left(r, r^{\prime}\right)=(-1)^{r}\left(\mathfrak{q}_{-}^{p_{+} r^{\prime}}+\mathfrak{q}_{-}^{-p_{+} r^{\prime}}\right) .
\end{aligned}
$$


These are roots of the respective polynomials $\psi_{+}$and $\psi_{-}$(see $\mathbf{B . 4 . 2}$ ). Moreover, as $\left(r, r^{\prime}\right)$ range over the set $\mathcal{I}$, all roots of $\psi_{+}$and $\psi_{-}$occur among the values taken by $\beta_{+}\left(r, r^{\prime}\right)$ and $\beta_{-}\left(r, r^{\prime}\right)$ respectively (not necessarily once). We then define

$$
\psi_{ \pm, r, r^{\prime}}(x)= \begin{cases}\frac{\psi_{ \pm}(x)}{x-\beta_{ \pm}\left(r, r^{\prime}\right)}, & \beta_{ \pm}\left(r, r^{\prime}\right)=2 \quad \text { or } \quad \beta_{ \pm}\left(r, r^{\prime}\right)=-2, \\ \frac{\psi_{ \pm}(x)}{\left(x-\beta_{ \pm}\left(r, r^{\prime}\right)\right)^{2}} & \text { otherwise }\end{cases}
$$

and set

$$
\boldsymbol{w}_{ \pm}\left(r, r^{\prime}\right)= \begin{cases}0, & \beta_{ \pm}\left(r, r^{\prime}\right)=2 \quad \text { or } \quad \beta_{ \pm}\left(r, r^{\prime}\right)=-2, \\ \left(\boldsymbol{C}_{ \pm}-\beta_{ \pm}\left(r, r^{\prime}\right)\right) \psi_{ \pm, r, r^{\prime}}\left(\boldsymbol{C}_{ \pm}\right) & \text {otherwise }\end{cases}
$$

and

$$
\boldsymbol{e}_{ \pm}\left(r, r^{\prime}\right)=\frac{1}{\psi_{ \pm, r, r^{\prime}}\left(\beta_{ \pm}\left(r, r^{\prime}\right)\right)}\left(\psi_{ \pm, r, r^{\prime}}\left(\boldsymbol{C}_{ \pm}\right)-\frac{\psi_{ \pm, r, r^{\prime}}^{\prime}\left(\beta_{ \pm}\left(r, r^{\prime}\right)\right)}{\psi_{ \pm, r, r^{\prime}}\left(\beta_{ \pm}\left(r, r^{\prime}\right)\right)} \boldsymbol{w}_{ \pm}\left(r, r^{\prime}\right)\right),
$$

where $\psi_{ \pm, r, r^{\prime}}^{\prime}(x)=\partial \psi_{ \pm, r, r^{\prime}}(x) / \partial x$.

Then

$$
\boldsymbol{e}\left(r, r^{\prime}\right)=\boldsymbol{e}_{+}\left(r, r^{\prime}\right) \boldsymbol{e}_{-}\left(r, r^{\prime}\right), \quad\left(r, r^{\prime}\right) \in \mathcal{I},
$$

are the $\frac{1}{2}\left(p_{+}+1\right)\left(p_{-}+1\right)$ primitive idempotents in the center.

The constants $\psi_{ \pm, r, r^{\prime}}\left(\beta_{ \pm}\left(r, r^{\prime}\right)\right)$ involved in the normalization factors are easily found explicitly. For this, we recall that the Chebyshev polynomials are eigenfunctions of a second-order differential operator,

$$
\left(\left(x^{2}-4\right) \frac{\partial^{2}}{\partial x^{2}}+3 x \frac{\partial}{\partial x}+1\right) U_{s}(x)=s^{2} U_{s}(x) .
$$

Whenever $\beta_{ \pm}\left(r, r^{\prime}\right) \neq 2$ and $\beta_{ \pm}\left(r, r^{\prime}\right) \neq-2$, i.e., $a=\beta_{ \pm}\left(r, r^{\prime}\right)$ is a multiplicity-2 root of $\psi_{ \pm}(x)$ in 2.2.5, we use (B.5) and (4.23) to find

$$
\left(a^{2}-4\right) \psi_{ \pm, r, r^{\prime}}(a)=4 p_{ \pm}^{2}+2 p_{ \pm} a U_{2 p_{ \pm}}(a)
$$

and hence

$$
\psi_{+, r, r^{\prime}}\left(\beta_{+}\left(r, r^{\prime}\right)\right)=\frac{4 p_{+}^{2}}{\left(\mathfrak{q}_{+}^{p_{-} r}-\mathfrak{q}_{+}^{-p_{-} r}\right)^{2}}, \quad \psi_{-, r, r^{\prime}}\left(\beta_{-}\left(r, r^{\prime}\right)\right)=\frac{4 p_{-}^{2}}{\left(\mathfrak{q}_{-}^{p_{+} r^{\prime}}-\mathfrak{q}_{-}^{-p_{+} r^{\prime}}\right)^{2}} .
$$

Also, if $\beta_{ \pm}\left(r, r^{\prime}\right)=2$, then

$$
\psi_{ \pm, r, r^{\prime}}\left(\beta_{ \pm}\left(r, r^{\prime}\right)\right)=\frac{4 p_{ \pm}^{2}}{3}+\frac{4 p_{ \pm}}{3} U_{2 p_{ \pm}}(2)=\frac{4 p_{ \pm}^{2}}{3}+\frac{8 p_{ \pm}^{2}}{3}=4 p_{ \pm}^{2},
$$

and if $\beta_{ \pm}\left(r, r^{\prime}\right)=-2$, then

$$
\psi_{ \pm, r, r^{\prime}}\left(\beta_{ \pm}\left(r, r^{\prime}\right)\right)=-\frac{4 p_{ \pm}^{2}}{3}+\frac{4 p_{ \pm}}{3} U_{2 p_{ \pm}}(-2)=-4 p_{ \pm}^{2} .
$$


To construct the canonical nilpotent elements, we next introduce the operators 9

$$
\begin{aligned}
& \boldsymbol{\pi}^{\uparrow}\left(r, r^{\prime}\right)=\frac{1}{2 p_{+} p_{-}} \sum_{j=0}^{2 p_{+} p_{-}-1} \sum_{\substack{a=-r+1 \\
\text { step }=2}}^{r-1} \sum_{\substack{a^{\prime}=-r^{\prime}+1 \\
\text { step }=2}}^{r^{\prime}-1} \mathfrak{q}_{+}^{-a j} \mathfrak{q}_{-}^{-a^{\prime} j} K^{j} \\
& \boldsymbol{\pi}^{\leftarrow}\left(r, r^{\prime}\right)=\frac{1}{2 p_{+} p_{-}} \sum_{j=0}^{2 p_{+} p_{-}-1} \sum_{\substack{a=-r+1 \\
\text { step }=2}}^{r-1} \sum_{\substack{a^{\prime}=-p_{-}+r^{\prime}+1 \\
\text { step }=2}}^{p_{-}-r^{\prime}-1}(-1)^{j} \mathfrak{q}_{+}^{-a j} \mathfrak{q}_{-}^{-a^{\prime} j} K^{j} \\
& \boldsymbol{\pi}^{\rightarrow}\left(r, r^{\prime}\right)=\frac{1}{2 p_{+} p_{-}} \sum_{j=0}^{2 p_{+} p_{-}-1} \sum_{\substack{a=-p_{+}+r+1 \\
\text { step }=2}}^{p_{+}-r-1} \sum_{\substack{a^{\prime}=-r^{\prime}+1 \\
\text { step }=2}}^{r^{\prime}-1}(-1)^{j} \mathfrak{q}_{+}^{-a j} \mathfrak{q}_{-}^{-a^{\prime} j} K^{j} \\
& \boldsymbol{\pi}^{\downarrow}\left(r, r^{\prime}\right)=\frac{1}{2 p_{+} p_{-}} \sum_{j=0}^{2 p_{+} p_{-}-1} \sum_{\substack{a=-p_{+}+r+1 \\
\text { step }=2}}^{p_{+}-r-1} \sum_{a^{\prime}=-p_{-}+r^{\prime}+1}^{p_{\text {step }=2}} \mathfrak{q}_{+}^{-a j} \mathfrak{q}_{-}^{-a^{\prime} j} K^{j} .
\end{aligned}
$$

These are pairwise orthogonal projection operators. We also note that

$$
\boldsymbol{\pi}^{\uparrow}\left(r, r^{\prime}\right)+\boldsymbol{\pi}^{\leftarrow}\left(r, r^{\prime}\right)+\boldsymbol{\pi}^{\rightarrow}\left(r, r^{\prime}\right)+\boldsymbol{\pi}^{\downarrow}\left(r, r^{\prime}\right)=\frac{1}{2}\left(\mathbf{1}+(-1)^{p_{-}(r-1)+p_{+}\left(r^{\prime}-1\right)} K^{p_{+} p_{-}}\right)
$$

and

$$
\boldsymbol{\pi}^{\leftarrow}\left(r, p_{-}\right)=\boldsymbol{\pi}^{\rightarrow}\left(p_{+}, r^{\prime}\right)=\boldsymbol{\pi}^{\downarrow}\left(r, p_{-}\right)=\boldsymbol{\pi}^{\downarrow}\left(p_{+}, r^{\prime}\right)=0 .
$$

For $\left(r, r^{\prime}\right) \in \mathcal{I}_{1}$ (see (1.9)), we use the above projectors to define the central elements

$$
\begin{aligned}
& \boldsymbol{v}^{\nearrow}\left(r, r^{\prime}\right)=\boldsymbol{e}_{+}\left(r, r^{\prime}\right) \boldsymbol{w}_{-}\left(r, r^{\prime}\right)\left(\boldsymbol{\pi}^{\uparrow}\left(r, r^{\prime}\right)+\boldsymbol{\pi}^{\rightarrow}\left(r, r^{\prime}\right)\right), \\
& \boldsymbol{v}^{\swarrow}\left(r, r^{\prime}\right)=\boldsymbol{e}_{+}\left(r, r^{\prime}\right) \boldsymbol{w}_{-}\left(r, r^{\prime}\right)\left(\boldsymbol{\pi}^{\leftarrow}\left(r, r^{\prime}\right)+\boldsymbol{\pi}^{\downarrow}\left(r, r^{\prime}\right)\right), \\
& \boldsymbol{v}^{\nwarrow}\left(r, r^{\prime}\right)=\boldsymbol{w}_{+}\left(r, r^{\prime}\right) \boldsymbol{e}_{-}\left(r, r^{\prime}\right)\left(\boldsymbol{\pi}^{\uparrow}\left(r, r^{\prime}\right)+\boldsymbol{\pi}^{\leftarrow}\left(r, r^{\prime}\right)\right), \\
& \boldsymbol{v}^{\searrow}\left(r, r^{\prime}\right)=\boldsymbol{w}_{+}\left(r, r^{\prime}\right) \boldsymbol{e}_{-}\left(r, r^{\prime}\right)\left(\boldsymbol{\pi}^{\rightarrow}\left(r, r^{\prime}\right)+\boldsymbol{\pi}^{\downarrow}\left(r, r^{\prime}\right)\right) .
\end{aligned}
$$

Clearly, their nonzero products are those in (2.22), with

$$
\begin{aligned}
\boldsymbol{w}^{\uparrow}\left(r, r^{\prime}\right) & =\boldsymbol{w}_{+}\left(r, r^{\prime}\right) \boldsymbol{w}_{-}\left(r, r^{\prime}\right) \boldsymbol{\pi}^{\uparrow}\left(r, r^{\prime}\right), \\
\boldsymbol{w}^{\rightarrow}\left(r, r^{\prime}\right) & =\boldsymbol{w}_{+}\left(r, r^{\prime}\right) \boldsymbol{w}_{-}\left(r, r^{\prime}\right) \boldsymbol{\pi}^{\rightarrow}\left(r, r^{\prime}\right), \\
\boldsymbol{w}^{\leftarrow}\left(r, r^{\prime}\right) & =\boldsymbol{w}_{+}\left(r, r^{\prime}\right) \boldsymbol{w}_{-}\left(r, r^{\prime}\right) \boldsymbol{\pi}^{\leftarrow}\left(r, r^{\prime}\right), \\
\boldsymbol{w}^{\downarrow}\left(r, r^{\prime}\right) & =\boldsymbol{w}_{+}\left(r, r^{\prime}\right) \boldsymbol{w}_{-}\left(r, r^{\prime}\right) \boldsymbol{\pi}^{\downarrow}\left(r, r^{\prime}\right) .
\end{aligned}
$$

The idempotent $\boldsymbol{e}\left(r, r^{\prime}\right)$ acts as identity on each of these 8 elements. This gives $(1+8)$. $\frac{1}{2}\left(p_{+}-1\right)\left(p_{-}-1\right)$ central elements, labeled by $\left(r, r^{\prime}\right) \in \mathcal{I}_{1}$. In addition, for $1 \leqslant r \leqslant p_{+}-1$, there are the central radical elements

$$
\begin{aligned}
\boldsymbol{v}^{\uparrow}\left(r, p_{-}\right) & =\boldsymbol{w}_{+}\left(r, p_{-}\right) \boldsymbol{e}_{-}\left(r, p_{-}\right) \boldsymbol{\pi}^{\uparrow}\left(r, p_{-}\right), \\
\boldsymbol{v}^{\rightarrow}\left(r, p_{-}\right) & =\boldsymbol{w}_{+}\left(r, p_{-}\right) \boldsymbol{e}_{-}\left(r, p_{-}\right) \boldsymbol{\pi}^{\rightarrow}\left(r, p_{-}\right),
\end{aligned}
$$

\footnotetext{
${ }^{9}$ The notation is correlated with the top, left, right, and bottom modules in (2.7); these $\pi$ operators project on the weights (i.e., the eigenvalues of $K$ ) occurring in the respective irreducible modules.
} 
on which $\boldsymbol{e}\left(r, p_{-}\right)$acts as identity (the total of $(1+2) \cdot\left(p_{+}-1\right)$ central elements), and for $1 \leqslant r^{\prime} \leqslant p_{-}-1$, there are the central radical elements

$$
\begin{aligned}
\boldsymbol{v}^{\uparrow}\left(p_{+}, r^{\prime}\right) & =\boldsymbol{e}_{+}\left(p_{+}, r^{\prime}\right) \boldsymbol{w}_{-}\left(p_{+}, r^{\prime}\right) \boldsymbol{\pi}^{\uparrow}\left(p_{+}, r^{\prime}\right), \\
\boldsymbol{v}^{\leftarrow}\left(p_{+}, r^{\prime}\right) & =\boldsymbol{e}_{+}\left(p_{+}, r^{\prime}\right) \boldsymbol{w}_{-}\left(p_{+}, r^{\prime}\right) \boldsymbol{\pi}^{\leftarrow}\left(p_{+}, r^{\prime}\right),
\end{aligned}
$$

on which $\boldsymbol{e}\left(p_{+}, r^{\prime}\right)$ acts as identity (the total of $(1+2) \cdot\left(p_{-}-1\right)$ central elements).

\section{B.4. Some $\mathfrak{g}_{p_{+}, p_{-}}$calculations.}

B.4.1. Proof of 2.3.4. Here, we prove about a quarter of the relations in 2.3.4 by establishing a similar statement for $\gamma\left(r, r^{\prime}\right)$ with $\alpha^{\uparrow, \downarrow}=\alpha^{\downarrow, \bullet}=\beta^{\downarrow, \bullet}=\beta^{\uparrow, \bullet}=0$, in which case the trace actually restricts to the submodule $\mathcal{P}_{r, r^{\prime}}^{+,+} \oplus \mathcal{P}_{p_{+}-r, r^{\prime}}^{-,+} \subset \mathbb{P}_{r, r^{\prime}}$ (see the definition of the modules $\mathcal{P}_{r, r^{\prime}}^{ \pm, \pm}$in $\mathbf{\text { A.1 }}$ ), and which eventually (after we ensure that $\alpha^{\uparrow, \uparrow}=\alpha^{\uparrow, \rightarrow}$ ) gives $\gamma^{\not{x}}\left(r, r^{\prime}\right)$ in (2.16).

We thus temporarily write $\gamma^{\pi /}\left(r, r^{\prime}\right)$ for $\gamma\left(r, r^{\prime}\right)$ as defined in (2.15) in the case where $\alpha^{\uparrow, \downarrow}=\alpha^{\downarrow, \bullet}=\beta^{\downarrow \bullet \bullet}=\beta^{\uparrow, \bullet}=0$. It is a $q$-character if and only if (see (2.8)

$$
0=\gamma^{\not{K}}(x y)-\gamma^{\not{\not}}\left(S^{2}(y) x\right) \equiv \operatorname{Tr}_{\mathbb{P}_{r, r^{\prime}}}\left(\boldsymbol{g}^{-1} x\left[y, \sigma^{\not{K}}\right]\right),
$$

where, as we have just noted,

$$
\operatorname{Tr}_{\mathbb{P}_{r, r^{\prime}}}\left(\boldsymbol{g}^{-1} x\left[y, \sigma^{\mathscr{K}}\right]\right)=\operatorname{Tr}_{\mathcal{P}_{r, r^{\prime}}^{+,+} \oplus \mathcal{P}_{p_{+}-,, r^{\prime}}}\left(\boldsymbol{g}^{-1} x\left[y, \sigma^{\mathscr{K}}\right]\right)
$$

It now follows from the formulas in A.1 that $\left[K, \sigma^{\not{K}}\left(r, r^{\prime}\right)\right]=0$ and the other commutation relations in $\mathcal{P}_{r, r^{\prime}}^{+,+}$are given by

$$
\begin{aligned}
& {\left[e_{+}, \sigma^{\mathscr{K}}\left(r, r^{\prime}\right)\right] \mathbf{b}_{n, n^{\prime}}^{\downarrow, \uparrow}= \begin{cases}\alpha^{\uparrow, \uparrow} \mathbf{b}_{n-1, n^{\prime}}^{\downarrow, \uparrow}, & 1 \leqslant n \leqslant r-1, \\
\alpha^{\uparrow, \uparrow} \mathbf{b}_{p_{+}-r-1, n^{\prime}}^{\leftarrow,}, & n=0,\end{cases} } \\
& {\left[e_{+}, \sigma^{\mathscr{K}}\left(r, r^{\prime}\right)\right] \mathbf{b}_{n, n^{\prime}}^{\uparrow, \uparrow}= \begin{cases}-\alpha^{\uparrow, \uparrow} \mathbf{b}_{n-1, n^{\prime}}^{\uparrow, \uparrow}, & 1 \leqslant n \leqslant r-1, \\
0, & n=0,\end{cases} } \\
& {\left[e_{+}, \sigma^{\not{K}}\left(r, r^{\prime}\right)\right] \mathbf{b}_{k, k^{\prime}}^{\rightarrow, \uparrow}= \begin{cases}0, & 1 \leqslant k \leqslant p_{+}-r-1, \\
-\alpha^{\uparrow, \uparrow} \mathbf{b}_{r-1, k^{\prime}}^{\uparrow, \uparrow}, & k=0,\end{cases} } \\
& {\left[e_{+}, \sigma^{\not{K}}\left(r, r^{\prime}\right)\right] \mathbf{b}_{k, k^{\prime}}^{\leftarrow, \uparrow}=0, \quad 1 \leqslant k \leqslant p_{+}-r-1 .}
\end{aligned}
$$

Writing the trace operation as

$$
\begin{aligned}
\operatorname{Tr}_{\mathcal{P}_{r, r^{\prime}}^{+,+}}(x)=\sum_{n=1}^{r-1} \sum_{n^{\prime}=0}^{r^{\prime}-1}\left\langle\mathrm{~b}_{n, n^{\prime}}^{\uparrow, \uparrow}|x| \mathbf{b}_{n, n^{\prime}}^{\uparrow, \uparrow}\right\rangle & +\sum_{n=1}^{p+-r-1} \sum_{n^{\prime}=0}^{r^{\prime}-1}\left\langle\mathrm{~b}_{n, n^{\prime}}^{\leftarrow, \uparrow}|x| \mathbf{b}_{n, n^{\prime}}^{\leftarrow, \uparrow}\right\rangle \\
& +\sum_{n=1}^{p_{+}-r-1} \sum_{n^{\prime}=0}^{r^{\prime}-1}\left\langle\mathrm{~b}_{n, n^{\prime}}^{\rightarrow, \uparrow}|x| \mathbf{b}_{n, n^{\prime}}^{\rightarrow, \uparrow}\right\rangle+\sum_{n=1}^{r-1} \sum_{n^{\prime}=0}^{r^{\prime}-1}\left\langle\mathbf{b}_{n, n^{\prime}}^{\downarrow, \uparrow}|x| \mathrm{b}_{n, n^{\prime}}^{\downarrow, \uparrow}\right\rangle,
\end{aligned}
$$


we then have

$\operatorname{Tr}_{\mathcal{P}_{r, r^{\prime}}^{+,+}}\left(\boldsymbol{g}^{-1} x\left[e_{+}, \sigma^{\mathscr{K}}\right]\right)=\alpha^{\uparrow, \uparrow} \sum_{n^{\prime}=0}^{r^{\prime}-1}\left\langle\mathbf{b}_{0, n^{\prime}}^{\downarrow, \uparrow}\left|\boldsymbol{g}^{-1} x\right| \mathbf{b}_{p_{+}-r-1, n^{\prime}}^{\leftarrow, \uparrow}\right\rangle-\alpha^{\uparrow, \uparrow} \sum_{n^{\prime}=0}^{r^{\prime}-1}\left\langle\mathrm{~b}_{0, n^{\prime}}^{\rightarrow, \uparrow}\left|\boldsymbol{g}^{-1} x\right| \mathbf{b}_{r-1, n^{\prime}}^{\uparrow, \uparrow}\right\rangle$

and, similarly,

$$
\begin{aligned}
\operatorname{Tr}_{\mathcal{P}_{p_{+}^{-},+, r^{\prime}}}\left(\boldsymbol{g}^{-1} x\left[e_{+}, \sigma^{\mathscr{K}}\right]\right)= \\
\quad=\alpha^{\uparrow, \rightarrow} \sum_{n^{\prime}=0}^{r^{\prime}-1}\left\langle\mathbf{b}_{0, n^{\prime}}^{\downarrow, \rightarrow}\left|\boldsymbol{g}^{-1} x\right| \mathbf{b}_{r-1, n^{\prime}}^{\leftarrow, \rightarrow}\right\rangle-\alpha^{\uparrow, \rightarrow} \sum_{n^{\prime}=0}^{r^{\prime}-1}\left\langle\mathbf{b}_{0, n^{\prime}}^{\rightarrow, \rightarrow}\left|\boldsymbol{g}^{-1} x\right| \mathbf{b}_{p_{+}-r-1, n^{\prime}}^{\uparrow, \rightarrow}\right\rangle .
\end{aligned}
$$

Summing these two contributions of the modules $\mathcal{P}_{r, r^{\prime}}^{+,+}$and $\mathcal{P}_{p_{+}-, r, r^{\prime}}^{-,+}$and using the obvious correspondence between the bases in the respective composition factors in $\mathcal{P}_{r, r^{\prime}}^{+,+}$and $\mathcal{P}_{p_{+}-r, r^{\prime}}^{-,+}$, we immediately obtain that the trace in (2.11) with $y=e_{+}$vanishes if and only if $\alpha^{\uparrow, \uparrow}=\alpha^{\uparrow, \rightarrow}$.

Similar calculations for the other generators $\left(f_{+}, e_{-}\right.$, and $\left.f_{-}\right)$give no more conditions for $\gamma^{\not{K}}\left(r, r^{\prime}\right)$ to be a $q$-character. This completes the proof in the simpler case of $\gamma^{\not{L}}$. The case of $\gamma^{\Uparrow}$ is equally uneventful but somewhat lengthier.

B.4.2. Casimir operators. In the calculations, we often use the well-known quantum$s \ell(2)$ identity (see, e.g., [27])

$$
f_{+}^{m} e_{+}^{m}=(-1)^{m} \prod_{s=0}^{m-1} \frac{\boldsymbol{C}_{+}+\mathfrak{q}_{+}^{p_{-}(2 s+1)} K_{+}+\mathfrak{q}_{+}^{-p_{-}(2 s+1)} K_{+}^{-1}}{\left(\mathfrak{q}_{+}^{p_{-}}-\mathfrak{q}_{+}^{-p_{-}}\right)^{2}}, \quad m<p_{+},
$$

where the element

$$
\boldsymbol{C}_{+}=-\mathfrak{q}_{+}^{p_{-}} K_{+}^{-1}-\mathfrak{q}_{+}^{-p_{-}} K_{+}-\left(q_{+}^{p_{-}}-q_{+}^{-p_{-}}\right)^{2} e_{+} f_{+}
$$

is central in $\mathfrak{g}_{p_{+}, p_{-}}$and therefore acts by the same eigenvalue on all vectors in each irreducible representation: using (2.6), we readily calculate

$$
\left.\boldsymbol{C}_{+}\right|_{\mathrm{X}_{r, r^{\prime}}^{\alpha}}=\alpha^{p_{-}}(-1)^{r^{\prime}}\left(\mathfrak{q}_{+}^{p_{-} r}+\mathfrak{q}_{+}^{-p_{-} r}\right)
$$

Similarly,

$$
e_{-}^{m^{\prime}} f_{-}^{m^{\prime}}=(-1)^{m^{\prime}} \prod_{s^{\prime}=0}^{m^{\prime}-1} \frac{\boldsymbol{C}_{-}+\mathfrak{q}_{-}^{-p_{+}\left(2 s^{\prime}+1\right)} K_{-}+\mathfrak{q}_{-}^{p_{+}\left(2 s^{\prime}+1\right)} K_{-}^{-1}}{\left(\mathfrak{q}_{-}^{p_{+}}-q_{-}^{-p_{+}}\right)^{2}}, \quad m^{\prime}<p_{-},
$$

where

$$
\boldsymbol{C}_{-}=-\mathfrak{q}_{-}^{p_{+}} K_{-}^{-1}-\mathfrak{q}_{-}^{-p_{+}} K_{-}-\left(q_{-}^{p_{+}}-q_{-}^{-p_{+}}\right)^{2} e_{-} f_{-}
$$

is central and

$$
\left.\boldsymbol{C}_{-}\right|_{\mathrm{X}_{r, r^{\prime}}^{\alpha}}=\alpha^{p_{+}}(-1)^{r}\left(\mathfrak{q}_{-}^{p_{+} r^{\prime}}+\mathfrak{q}_{-}^{-p_{+} r^{\prime}}\right) .
$$


The elements $\boldsymbol{C}_{+}$satisfy the equations

$$
\psi_{+}\left(\boldsymbol{C}_{+}\right)=0, \quad \psi_{-}\left(\boldsymbol{C}_{-}\right)=0,
$$

where

$$
\psi_{ \pm}(x)=\prod_{r=0}^{p_{ \pm}-1}\left(x+\mathfrak{q}_{ \pm}^{p_{\mp} r}+\mathfrak{q}_{ \pm}^{-p_{\mp} r}\right)\left(x-\mathfrak{q}_{ \pm}^{p_{\mp} r}-\mathfrak{q}_{ \pm}^{-p_{\mp} r}\right)
$$

(another relation between $\boldsymbol{C}_{+}$and $\boldsymbol{C}_{-}$, characteristic of the $\mathfrak{g}_{p_{+}, p_{-}}$quantum group rather than of the quantum $s \ell(2)$, is given in (4.24)). We note that $\psi_{+}(x)$ (and similarly $\psi_{-}(x)$ ) has two roots of multiplicity 1 , which are \pm 2 , and all the other roots are of multiplicity 2 .

In finding decompositions of central elements as explained in B.2.2, we also need the action of $\boldsymbol{C}_{ \pm}$on some indecomposable modules. For example, the action of $\boldsymbol{C}_{+}$on $\mathcal{P}_{r, r^{\prime}}^{\alpha,+}$ (see A.1) can be written as

$$
\left.\boldsymbol{C}_{+}\right|_{\mathcal{P}_{r, r^{\prime}}^{\alpha,+}}=\alpha^{p_{-}}(-1)^{r^{\prime}}\left(\mathfrak{q}_{+}^{p_{-} r}+\mathfrak{q}_{+}^{-p_{-} r}\right)-\left(\mathfrak{q}_{+}^{p_{-}}-\mathfrak{q}_{+}^{-p_{-}}\right)^{2} \sum_{n, n^{\prime}} \mathrm{t}_{n, n^{\prime}}^{\downarrow} \frac{\partial}{\partial \mathrm{t}_{n, n^{\prime}}^{\uparrow}}
$$

in terms of the bases introduced in $\mathbf{A . 1}$. It now follows from (B.17) and (B.10) that

$$
f_{+}^{m} e_{+}^{m} \mathrm{t}_{a, a^{\prime}}^{\uparrow}=\prod_{s=0}^{m-1}\left(\mathrm{t}_{a, a^{\prime}}^{\downarrow} \frac{\partial}{\partial \mathrm{t}_{a, a^{\prime}}^{\uparrow}}+\alpha^{p_{-}}(-1)^{r^{\prime}-1}[s-a+r]_{+}[a-s]_{+}\right) \mathrm{t}_{a, a^{\prime}}^{\uparrow}, \quad m<p_{+} .
$$

Writing $f_{+}^{m} e_{+}^{m} \mathrm{t}_{a, a^{\prime}}^{\uparrow}=A_{\uparrow} \mathrm{t}_{a, a^{\prime}}^{\uparrow}+A_{\downarrow} \mathrm{t}_{a, a^{\prime}}^{\downarrow}$, we see that

$$
A_{\downarrow}=\alpha^{p_{-}(m-1)}(-1)^{(m-1)\left(r^{\prime}-1\right)}\left[x^{1}\right]\left(\mathcal{C}_{+, r, a}^{m}(x)\right),
$$

where we define the polynomials

$$
\mathfrak{C}_{ \pm, r, a}^{m}(x)=\prod_{s=0}^{m-1}\left(x+[s-a+r]_{ \pm}[a-s]_{ \pm}\right)
$$

and use $\left[x^{n}\right](f(x))$ to denote the coefficient at $x^{n}$.

Similar formulas can be easily written for the action of $\boldsymbol{C}_{-}$and for the action of $\boldsymbol{C}_{ \pm}$ on other indecomposable modules.

We note that

and

$$
\left[x^{0}\right]\left(\mathcal{C}_{ \pm, r, a}^{m}(x)\right)=\left([m]_{ \pm} !\right)^{2}\left[\begin{array}{c}
a \\
m
\end{array}\right]_{ \pm}\left[\begin{array}{c}
r-a+m-1 \\
m
\end{array}\right]_{ \pm}
$$

$$
\left[x^{1}\right]\left(\mathcal{C}_{+, 1, a}^{m}(x)\right)=(-1)^{m+1}[m]_{+} ![m-1]_{+} !\left[\begin{array}{c}
a-1 \\
a-m
\end{array}\right]_{+}^{2},
$$

with $\left[x^{1}\right]\left(\mathcal{C}_{+, 1,0}^{m}(x)\right)=(-1)^{m+1}[m]_{+} ![m-1]_{+}$! for $m \geqslant 1$, and

$$
\left[x^{1}\right]\left(\mathcal{C}_{+, p_{+}-1, a}^{m}(x)\right)=(-1)^{\left(p_{-}+1\right)(m+1)}[m-1]_{+} ![m]_{+} !\left[\begin{array}{c}
a \\
m-1
\end{array}\right]_{+}^{2} .
$$


B.4.3. Proof of 3.2.1. We begin with calculating the comultiplication of the cointegral as

$$
\begin{array}{r}
\Delta(\boldsymbol{\Lambda})=\zeta \sum_{r=0}^{p_{+}-1} \sum_{m=0}^{p_{+}-1} \sum_{s=0} \sum_{n=0}^{p_{-}-1} \sum_{\ell=0}^{p_{-}-1} \mathfrak{q}_{+}^{2 p_{+} p_{-}-1}{ }^{-p_{-}(m+r+1)(m+r+2)} \mathfrak{q}_{-}^{-p_{+}(n+s+1)(n+s+2)} \\
\times(-1)^{r+m+n+s} f_{+}^{p_{+}-r-1} e_{+}^{m} f_{-}^{n} e_{-}^{p_{-}-1-s} k^{2 \ell-2 p_{-}(m+1)+2 p_{+}(n+1)} \otimes \\
\otimes f_{+}^{r} e_{+}^{p_{+}-1-m} f_{-}^{p_{-}-1-n} e_{-}^{s} k^{2 \ell+2 p_{-}(r+1)-2 p_{+}(s+1)}
\end{array}
$$

and then evaluate the traces in the definition of $\widehat{\phi}^{ \pm}\left(r, r^{\prime}\right)$ using (B.10) $-($ B.15). This gives

$$
\widehat{\boldsymbol{\phi}}^{\alpha}\left(r, r^{\prime}\right)=\zeta \widehat{\boldsymbol{\phi}}_{(+)}^{\alpha}\left(r, r^{\prime}\right)\left(\mathbf{1}+\alpha^{p_{+} p_{-}}(-1)^{p_{+}\left(r^{\prime}-1\right)+p_{-}(r-1)} K^{p_{+} p_{-}}\right) \widehat{\boldsymbol{\phi}}_{(-)}^{\alpha}\left(r, r^{\prime}\right),
$$

where $1 \leqslant r \leqslant p_{+}$and $1 \leqslant r^{\prime} \leqslant p_{-}$, and

$$
\begin{aligned}
\widehat{\phi}_{(+)}^{\alpha}\left(r, r^{\prime}\right)=\sum_{m=0}^{p_{+}-1} \sum_{a=m}^{r-1} & \sum_{b=0}^{p_{+}-1}(-1)^{b\left(r^{\prime}-1\right)} \alpha^{b p_{-}}\left([m]_{+} !\right)^{2} \\
& \times \mathfrak{q}_{+}^{p_{-}(r-1-2 a)(b-m)}\left[\begin{array}{c}
r-a+m-1 \\
m
\end{array}\right]_{+}\left[\begin{array}{c}
a \\
m
\end{array}\right]_{+} f_{+}^{p_{+}-m-1} e_{+}^{p_{+}-m-1} K_{+}^{b-m}
\end{aligned}
$$

and

$$
\begin{aligned}
\widehat{\boldsymbol{\phi}}_{(-)}^{\alpha}\left(r, r^{\prime}\right) & =\sum_{m^{\prime}=0}^{p_{-}-1} \sum_{a^{\prime}=0}^{r^{\prime}-m^{\prime}-1} \sum_{b^{\prime}=0}^{p_{-}-1}(-1)^{b^{\prime}(r-1)} \alpha^{b^{\prime} p_{+}}\left(\left[m^{\prime}\right]_{-} !\right)^{2} \\
& \times \mathfrak{q}_{-}^{p_{+}\left(r^{\prime}-1-2 a^{\prime}-2 m^{\prime}\right)\left(b^{\prime}+m^{\prime}\right)}\left[\begin{array}{c}
r^{\prime}-a^{\prime}-1 \\
m^{\prime}
\end{array}\right]_{-}\left[\begin{array}{c}
a^{\prime}+m^{\prime} \\
m^{\prime}
\end{array}\right]_{-} f_{-}^{p_{-}-m^{\prime}-1} e_{-}^{p_{-}-m^{\prime}-1} K_{-}^{b^{\prime}+m^{\prime}} .
\end{aligned}
$$

As regards the central elements $\widehat{\phi}^{\nwarrow}\left(r, r^{\prime}\right)$ and $\widehat{\phi}^{\nwarrow \searrow}\left(r, r^{\prime}\right)$, we recall the nondiagonal action of $\boldsymbol{C}_{ \pm}$on indecomposable modules, see B.4.2. Straightforward calculations then give the following formulas:

$$
\widehat{\boldsymbol{\phi}}^{\not{x}}\left(r, r^{\prime}\right)=\boldsymbol{\omega}^{+}\left(r, r^{\prime}\right)-(-1)^{p_{-}} \boldsymbol{\omega}^{-}\left(p_{+}-r, r^{\prime}\right)
$$

for $1 \leqslant r \leqslant p_{+}-1$ and $1 \leqslant r^{\prime} \leqslant p_{-}$,

$$
\widehat{\boldsymbol{\phi}}^{\nwarrow \searrow}\left(r, r^{\prime}\right)=\overline{\boldsymbol{\omega}}^{+}\left(r, r^{\prime}\right)-(-1)^{p_{+}} \overline{\boldsymbol{\omega}}^{-}\left(r, p_{-}-r^{\prime}\right)
$$

for $1 \leqslant r \leqslant p_{+}$and $1 \leqslant r^{\prime} \leqslant p_{-}-1$, and

$$
\begin{aligned}
\widehat{\phi}^{\Uparrow}\left(r, r^{\prime}\right)=\Omega^{+}\left(r, r^{\prime}\right) & -(-1)^{p_{-}} \boldsymbol{\Omega}^{-}\left(p_{+}-r, r^{\prime}\right) \\
& -(-1)^{p_{+}} \boldsymbol{\Omega}^{-}\left(r, p_{-}-r^{\prime}\right)+(-1)^{p_{-}+p_{+}} \boldsymbol{\Omega}^{+}\left(p_{+}-r, p_{-}-r^{\prime}\right)
\end{aligned}
$$

for $\left(r, r^{\prime}\right) \in \mathcal{I}_{1}$, where

$$
\begin{aligned}
\boldsymbol{\omega}^{\alpha}\left(r, r^{\prime}\right)= & \zeta \frac{[r]_{+}}{\mathfrak{q}_{+}^{p_{-}-\mathfrak{q}_{+}^{-p_{-}}}} \sum_{m=0}^{p_{+}-1} \sum_{m^{\prime}=0}^{p_{-}-1} \sum_{a=0}^{r-1} \sum_{a^{\prime}=m^{\prime}}^{r^{\prime}-1} \sum_{\ell=0}^{2 p_{+} p_{-}-1} \alpha^{\ell-p_{-}}(-1)^{r^{\prime}-1} \\
& \left.\times \mathfrak{q}_{+}^{\left(\ell-p_{-} m\right)(r-1-2 a)} \mathfrak{q}_{-}^{\left(\ell+p_{+}\right.}\right)\left(r^{\prime}-1-2 a^{\prime}\right)\left[x^{1}\right]\left(\mathcal{C}_{+, r, a}^{m}(x)\right)\left[y^{0}\right]\left(\mathcal{C}_{-, r^{\prime}, a^{\prime}}^{m^{\prime}}(y)\right)
\end{aligned}
$$




$$
\begin{aligned}
& \times f_{+}^{p_{+}-1-m} e_{+}^{p_{+}-1-m} f_{-}^{p_{-}-1-m^{\prime}} e_{-}^{p_{-}-1-m^{\prime}} K^{\ell-p_{-} m+p_{+} m^{\prime}}, \\
& \overline{\boldsymbol{\omega}}^{\alpha}\left(r, r^{\prime}\right)=\zeta \frac{\left[r^{\prime}\right]_{-}}{\mathfrak{q}_{-}^{p_{+}}-\mathfrak{q}_{-}^{-p_{+}}} \sum_{m^{\prime}=0}^{p_{-}-1} \sum_{m=0}^{p_{+}-1} \sum_{\ell=0}^{2 p_{+} p_{-}-1} \sum_{a^{\prime}=0}^{r^{\prime}-1} \sum_{a=m}^{r-1} \alpha^{\ell-p_{+}}(-1)^{r-1} \\
& \times \mathfrak{q}_{-}^{\left(\ell-p_{+} m^{\prime}\right)\left(r^{\prime}-1-2 a^{\prime}\right)} \mathfrak{q}_{+}^{\left(\ell+p_{-} m\right)(r-1-2 a)}\left[x^{0}\right]\left(\mathrm{C}_{+, r, a}^{m}(x)\right)\left[y^{1}\right]\left(\mathrm{C}_{-, r^{\prime}, a^{\prime}}^{m^{\prime}}(y)\right) \\
& \times f_{-}^{p_{-}-1-m^{\prime}} e_{-}^{p_{-}-1-m^{\prime}} f_{+}^{p_{+}-1-m} e_{+}^{p_{+}-1-m} K^{\ell-p_{+} m^{\prime}+p_{-} m},
\end{aligned}
$$

and

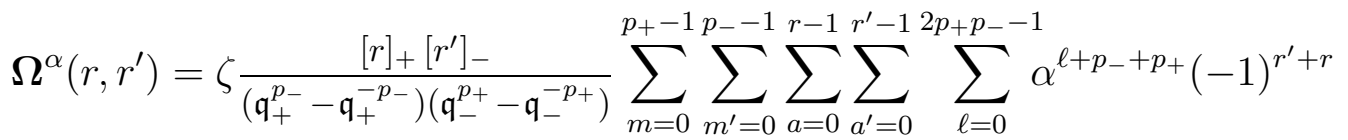

$$
\begin{aligned}
& \times \mathfrak{q}_{+}^{\left(\ell-p_{-} m\right)(r-1-2 a)} \mathfrak{q}_{-}^{\left(\ell-p_{+} m^{\prime}\right)\left(r^{\prime}-1-2 a^{\prime}\right)}\left[x^{1}\right]\left(\mathfrak{C}_{+, r, a}^{m}(x)\right)\left[y^{1}\right]\left(\mathcal{C}_{+, r^{\prime}, a^{\prime}}^{m^{\prime}}(y)\right) \\
& \times f_{+}^{p_{+}-1-m} e_{+}^{p_{+}-1-m} f_{-}^{p_{-}-1-m^{\prime}} e_{-}^{p_{-}-1-m^{\prime}} K^{\ell-p_{-} m-p_{+} m^{\prime}} .
\end{aligned}
$$

The rest of the proof (establishing the decomposition in terms of the canonical central elements) is by direct evaluation of the action of $\widehat{\phi}^{ \pm}\left(r, r^{\prime}\right), \widehat{\phi}^{\nwarrow}\left(r, r^{\prime}\right), \widehat{\phi}^{\nwarrow}\left(r, r^{\prime}\right)$, and $\widehat{\boldsymbol{\phi}}^{\uparrow}\left(r, r^{\prime}\right)$ on the projective modules, as described in B.2.2. The nondiagonal part of the action on the projective modules described in $\mathbf{A . 2}$ is related to the coefficients at the radical elements entering the decomposition with respect to the canonical central elements. The calculation yielding (3.10), (3.11), (3.12), (3.13), and (3.14) is very similar to the one given in [5], and we therefore omit it.

\section{REFERENCES}

[1] T. Kerler and V.V. Lyubashenko, Non-Semisimple Topological Quantum Field Theories for 3Manifolds with Corners, Springer Lecture Notes in Mathematics 1765, Springer Verlag (2001).

[2] J. Fuchs, S. Hwang, A.M. Semikhatov, and I.Yu. Tipunin, Nonsemisimple fusion algebras and the Verlinde formula, Commun. Math. Phys. 247 (2004) 713-742 [hep-th/0306274].

[3] M. Miyamoto, Modular invariance of vertex operator algebras satisfying $C_{2}$-cofiniteness, math.QA/ 0209101.

[4] Y.-Z. Huang, J. Lepowsky, and L. Zhang, A logarithmic generalization of tensor product theory for modules for a vertex operator algebra, math.QA/0311235.

[5] B.L. Feigin, A.M. Gainutdinov, A.M. Semikhatov, and I.Yu. Tipunin, Modular group representations and fusion in logarithmic conformal field theories and in the quantum group center, Commun. Math. Phys. 265 (2006) 47-93 [hep-th/0504093].

[6] M. Flohr and M.R. Gaberdiel, Logarithmic torus amplitudes, hep-th/0509075.

[7] B.L. Feigin, A.M. Gainutdinov, A.M. Semikhatov, and I.Yu. Tipunin, Kazhdan-Lusztig correspondence for the representation category of the triplet $W$-algebra in logarithmic CFT, math.QA/ 0512621.

[8] J. Fuchs, On non-semisimple fusion rules and tensor categories, hep-th/0602051.

[9] J. Cardy, Critical percolation in finite geometries, J. Phys. A25 (1992) L201-L206 [hep-th/9111026].

[10] G. Watts, A crossing probability for critical percolation in two dimensions, J. Phys. A29 (1996) L363 [cond-mat/9603167]. 
[11] H. Saleur and B. Duplantier, Exact determination of the percolation hull exponent in two dimensions, Phys. Rev. Lett. 58 (1987) 2325-2328.

[12] D. Kazhdan and G. Lusztig, Tensor structures arising from affine Lie algebras, I, J. Amer. Math. Soc. 6 (1993) 905-947; II, J. Amer. Math. Soc. 6 (1993) 949-1011; III, J. Amer. Math. Soc. 7 (1994) 335-381; IV, J. Amer. Math. Soc. 7 (1994) 383-453.

[13] G. Felder, BRST approach to minimal models, Nucl. Phys. B317 (1989) 215-236.

[14] P. Bouwknegt, J. McCarthy, K. Pilch, Fock space resolutions of the Virasoro highest weight modules with $c \leqslant 1$, Lett. Math. Phys. 23 (1991) 193-204 [hep-th/9108023].

[15] M.A.I. Flohr, On modular invariant partition functions of conformal field theories with logarithmic operators, Int. J. Mod. Phys. A11 (1996) 4147 [hep-th/9509166].

[16] M. Flohr, On Fusion Rules in Logarithmic Conformal Field Theories, Int. J. Mod. Phys. A12 (1997) 1943-1958 [hep-th/9605151].

[17] V. Lyubashenko, Invariants of 3-manifolds and projective representations of mapping class groups via quantum groups at roots of unity, Commun. Math. Phys. 172 (1995) 467-516 [hep-th/9405167]; Modular properties of ribbon abelian categories, Symposia Gaussiana, Proc. of the 2nd Gauss Symposium, Munich, 1993, Conf. A (Berlin, New York), Walter de Gruyter, (1995) 529-579 [hep-th/ 9405168]; Modular Transformations for Tensor Categories, J. Pure Applied Algebra 98 (1995) 279327.

[18] V. Lyubashenko and S. Majid, Braided groups and quantum Fourier transform, J. Algebra 166 (1994) 506-528.

[19] T. Kerler, Mapping class group action on quantum doubles, Commun. Math. Phys. 168 (1995) 353388 [hep-th/9402017].

[20] A. Lachowska, On the center of the small quantum group, math.QA/0107098.

[21] V.G. Drinfeld, On almost cocommutative Hopf algebras, Leningrad Math. J. 1 (1990) No. 2, 321-342.

[22] B.L. Feigin, A.M. Gainutdinov, A.M. Semikhatov, and I.Yu. Tipunin, Logarithmic extensions of minimal models: characters and modular transformations, Nucl. Phys. B 757 (2006) 303-343 [hep-th/ 0606196].

[23] J.L. Cardy, Nucl. Phys. B 324 (1989) 581-596.

[24] R.G. Larson and M.E. Sweedler, An associative orthogonal bilinear form for Hopf algebras, Amer. J. Math., 91 (1969) 75-94.

[25] D.E. Radford, The order of antipode of a finite-dimensional Hopf algebra is finite, Amer. J. Math 98 (1976) 333-335.

[26] C. Kassel, Quantum Groups, Springer-Verlag, New York, 1995.

[27] V. Chari and A. Pressley, A Guide to Quantum Groups, Cambridge University Press (1994).

[28] M.E. Sweedler, Hopf Algebras, Benjamin, New York, NY (1969).

[29] D.E. Radford, The trace function and Hopf algebras, J. Alg. 163 (1994) 583-622.

[30] N.Yu. Reshetikhin and M.A. Semenov-Tian-Shansky, Quantum R-matrices and factorization problems, J. Geom. Phys. 5 (1988) 533-550.

BLF: LANDAU InSTITUTE FOR TheORETICAL Physics

FEIGIN@MCCME.RU

AMS, AMG, IYT: LEBEDEV Physics InSTITUTE

AMS CSCI.LEBEDEV.RU, AZOT@MCCME.RU, TIPUNIN@TD.LPI •RU 